\title{
Developing new inexpensive room temperature ionic liquids with high thermal stability and a greener synthetic profile
}

Mahdi Ghorbani ${ }^{a, b *}$, Michela I. Simone ${ }^{a *}$

a. Discipline of Chemistry, University of Newcastle, Callaghan, NSW 2308, Australia.

b. Institute for Frontier Materials, Deakin University, 221 Burwood Highway, Burwood 3125, VIC, Australia.

General NMR experimental: all spectra were collected at room temperature on a Bruker Ascend $\mathrm{TM}$ 400, in $\mathrm{CDCl}_{3}$ and calibrated at $7.26 \mathrm{ppm}$ and $77.20 \mathrm{ppm}$ respectively for calibration of the ${ }^{1} \mathrm{H}$ - and ${ }^{13} \mathrm{C}-\mathrm{NMR}$ spectra.

\section{Table of content}

${ }^{1} \mathrm{H}$ - and ${ }^{13} \mathrm{C}-\mathrm{NMR}$ spectra for raw materials and products. 
Figure S1. ${ }^{1} \mathrm{H}-\mathrm{NMR}$ spectrum for starting material 1,8-diazabicyclo[5.4.0] undec-7-ene (DBU)
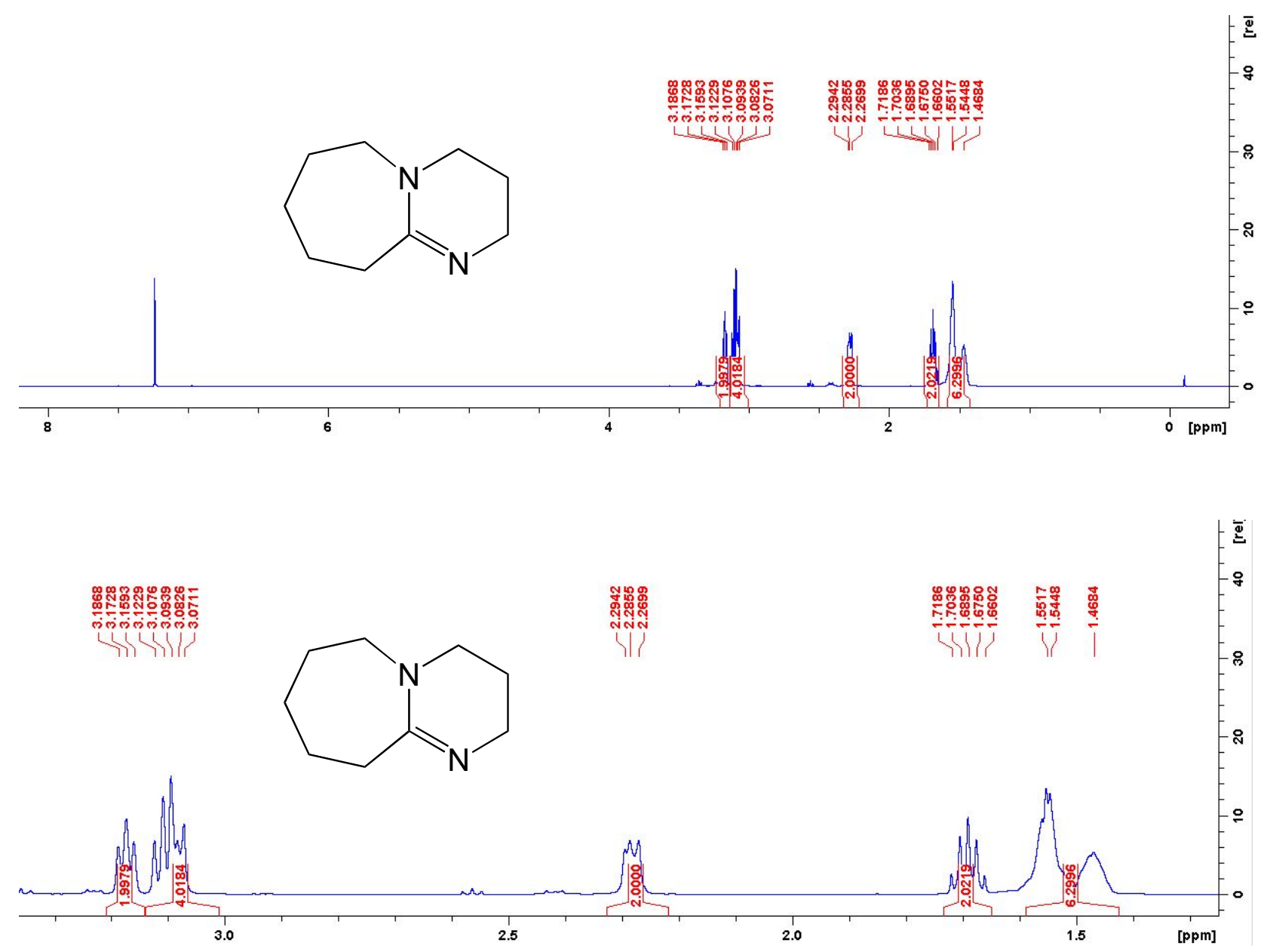

Figure S2. ${ }^{13} \mathrm{C}-\mathrm{NMR}$ spectrum for starting material 1,8-diazabicyclo[5.4.0]undec-7-ene (DBU)

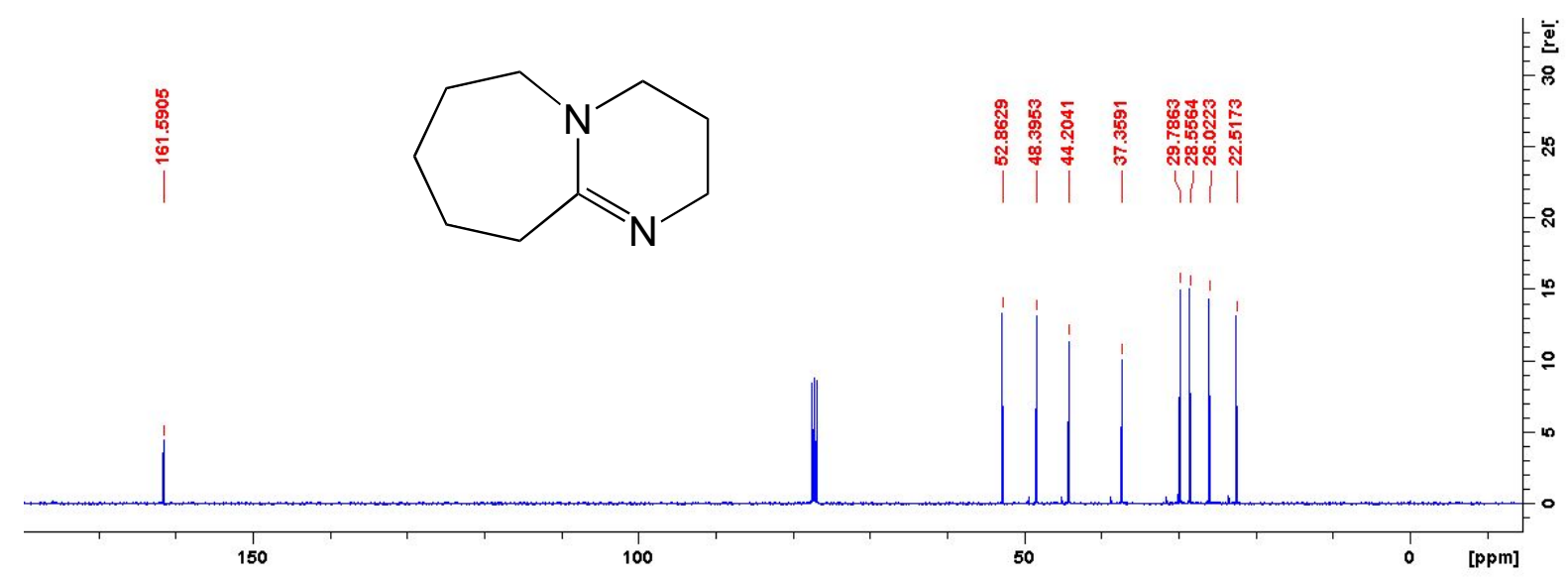


Figure S3. ${ }^{1} \mathrm{H}-\mathrm{NMR}$ spectrum for starting material chloromethyl trimethylsilane

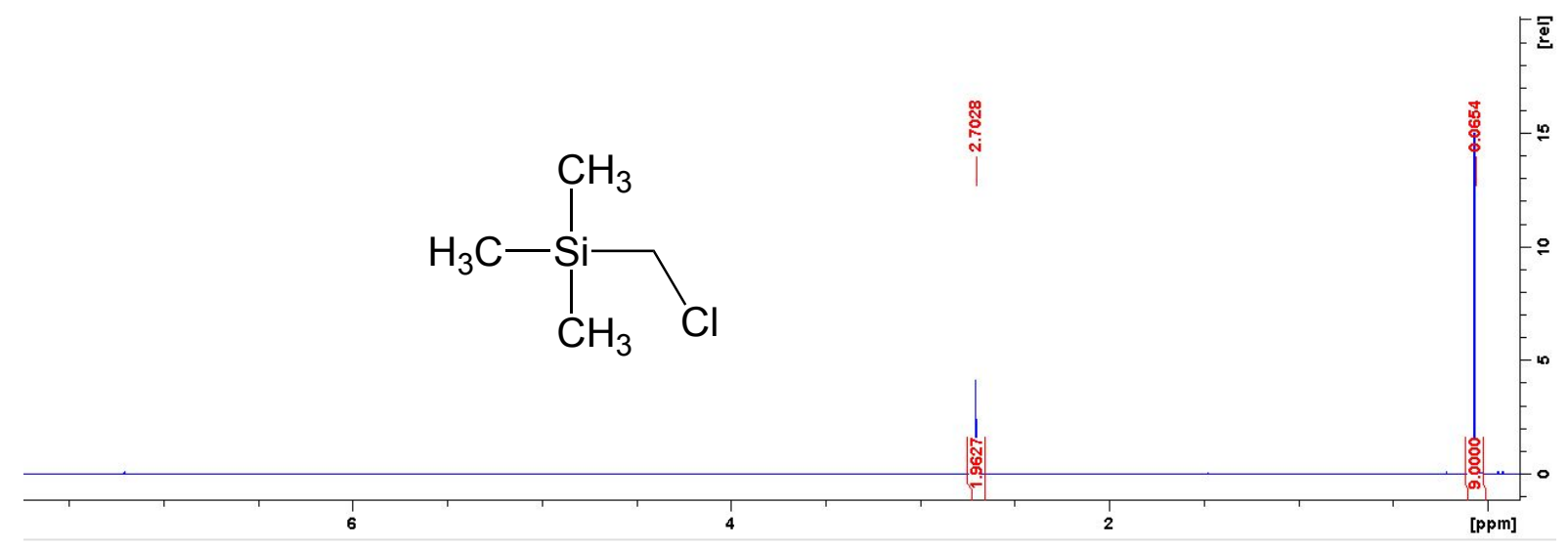

Figure S4. ${ }^{13} \mathrm{C}-\mathrm{NMR}$ spectrum for starting material chloromethyl trimethylsilane

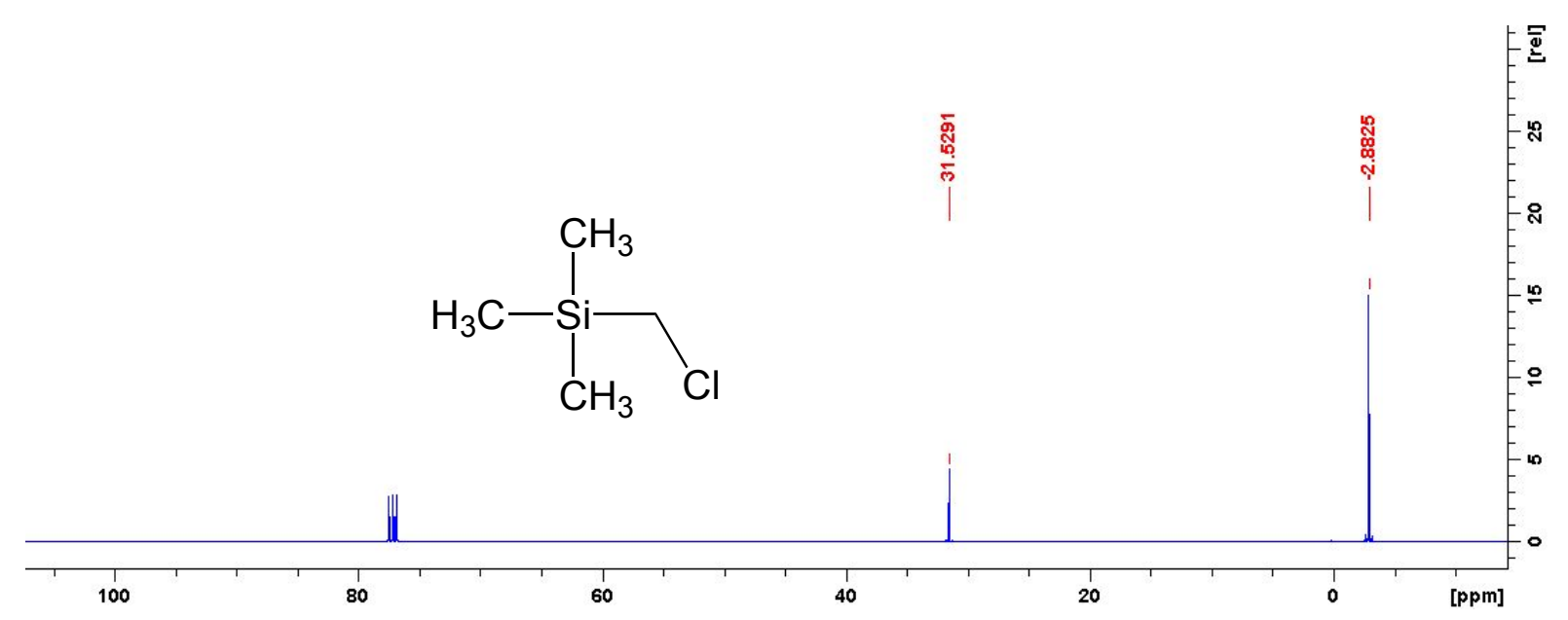

Figure S5. ${ }^{1} \mathrm{H}-\mathrm{NMR}$ spectrum for IL intermediate [DBU-M-TMSi] Cl

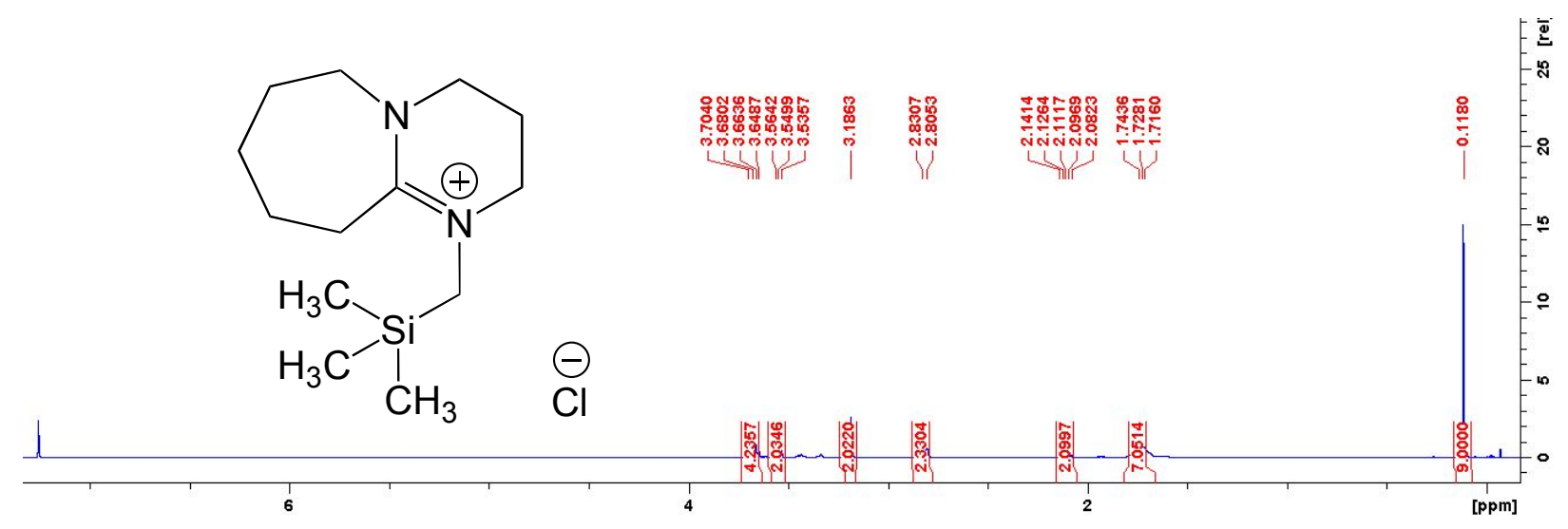




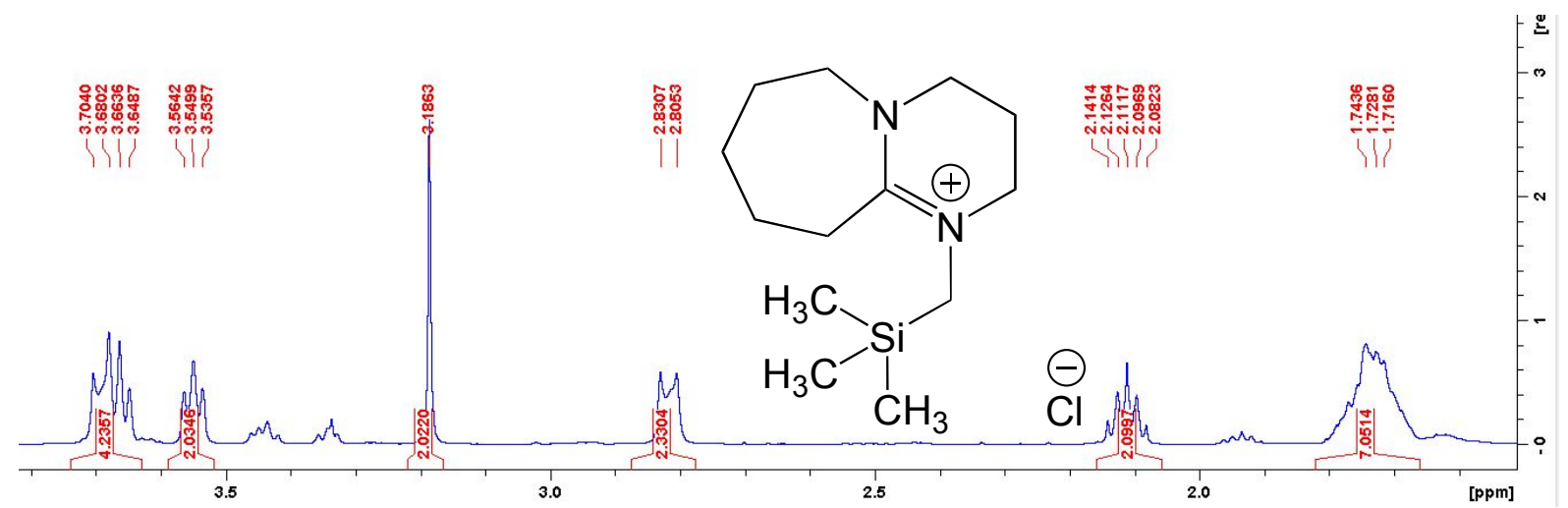

Figure S6. ${ }^{13} \mathrm{C}-\mathrm{NMR}$ spectrum for IL intermediate [DBU-M-TMSi] Cl
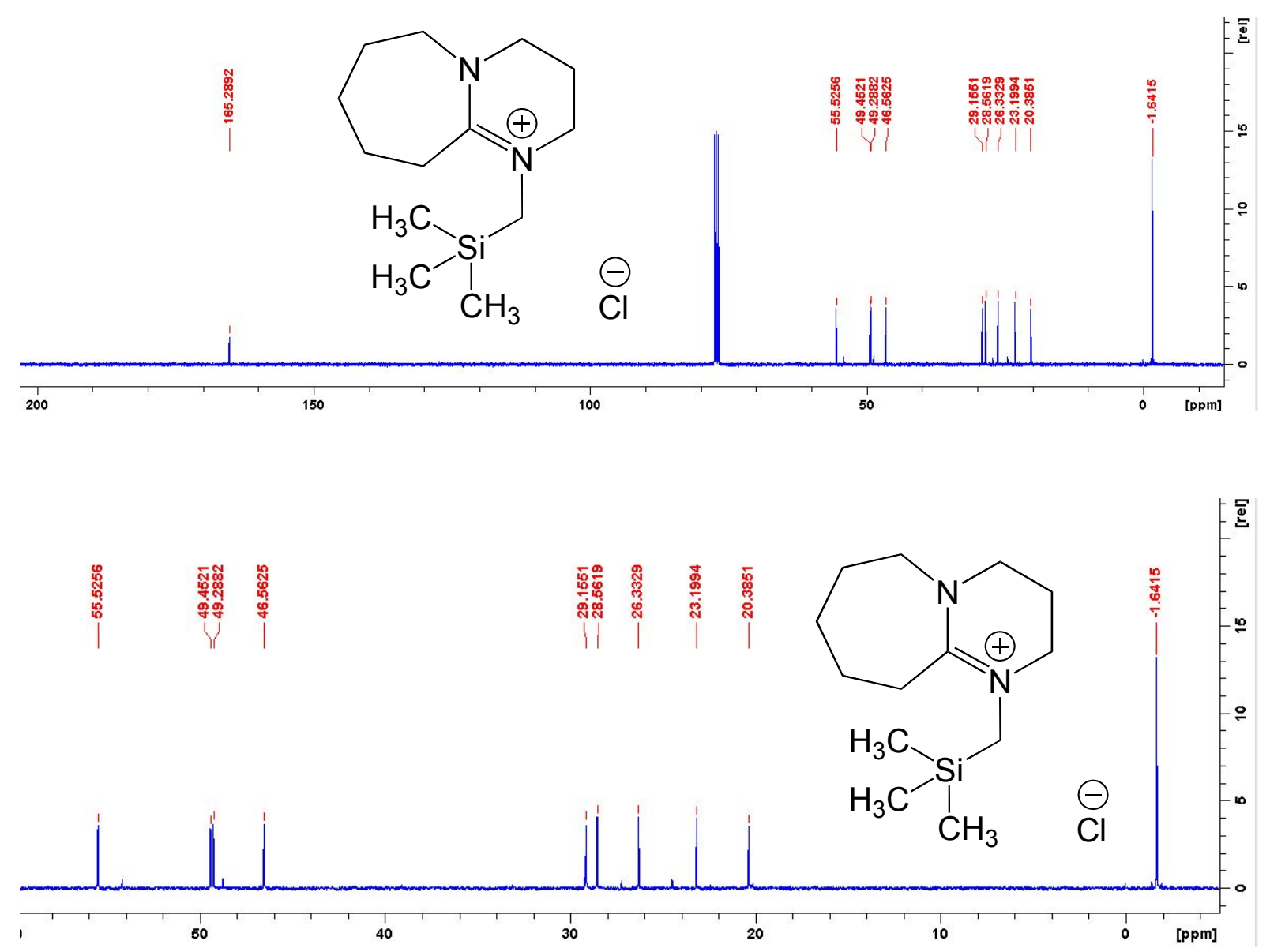
Figure S7. ${ }^{1} \mathrm{H}-\mathrm{NMR}$ spectrum for IL product [DBU-M-TMSi] $\mathrm{NTf}_{2}$
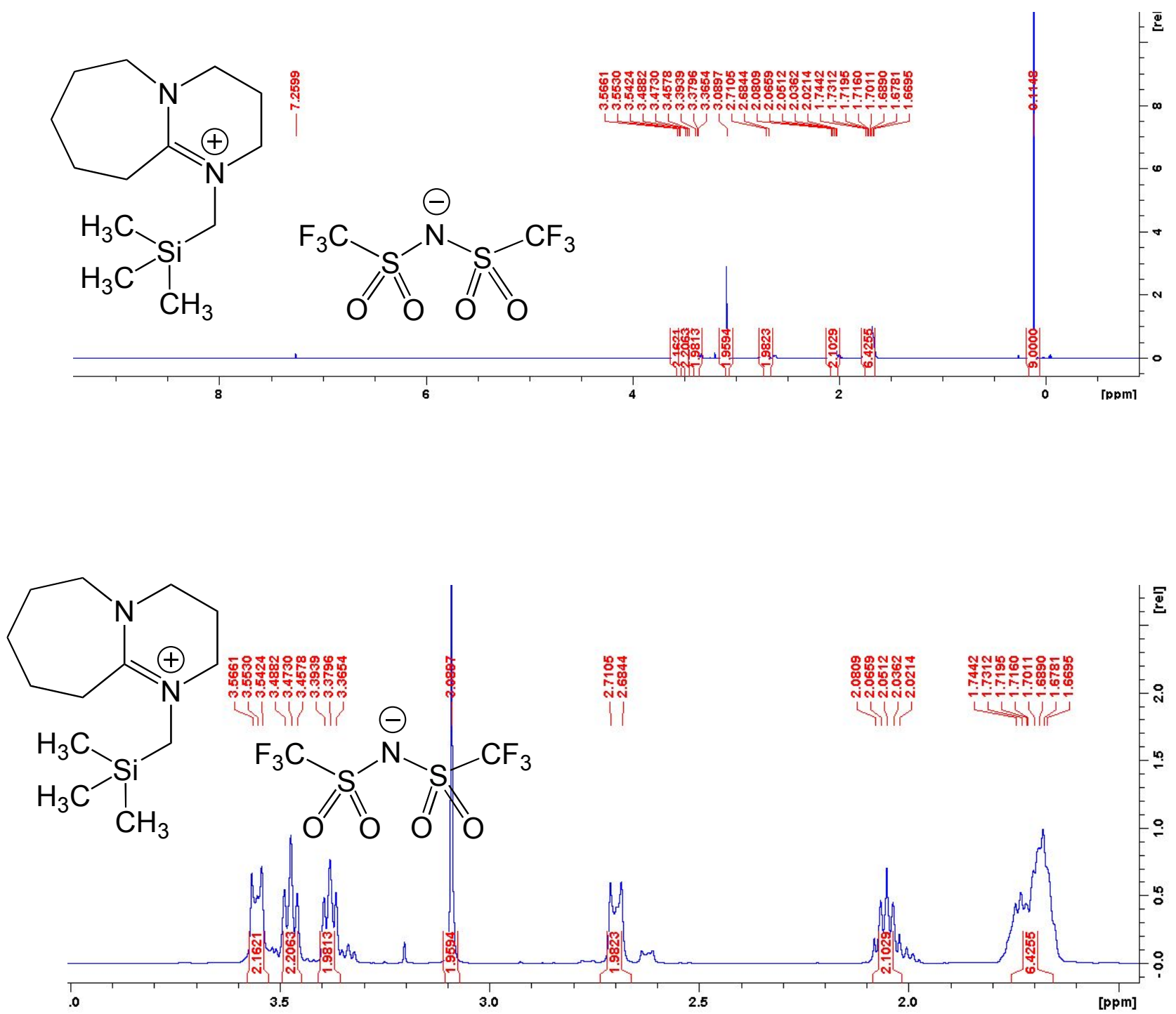

Figure S8. ${ }^{13} \mathrm{C}-\mathrm{NMR}$ spectrum for IL product [DBU-M-TMSi] $\mathrm{NTf}_{2}$

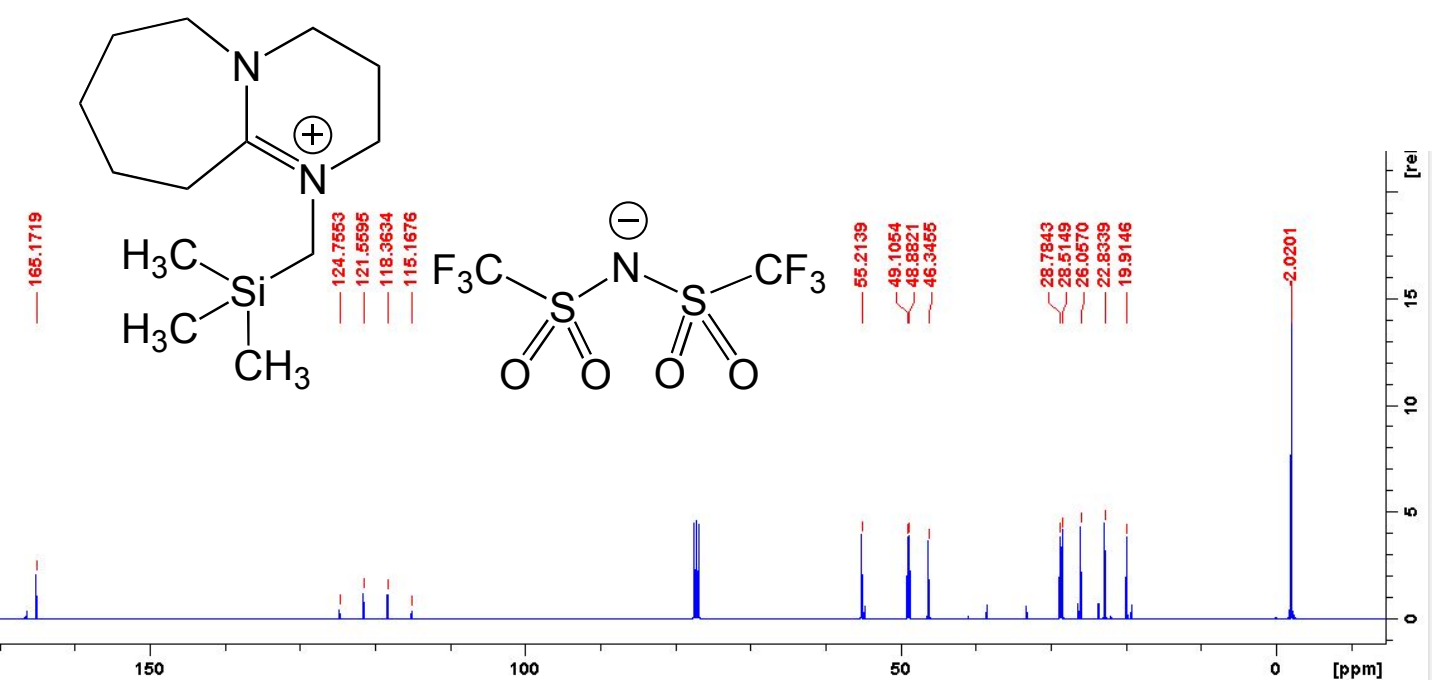


Figure S9. ${ }^{19} \mathrm{~F}-\mathrm{NMR}$ spectrum for IL product [DBU-M-TMSi] $\mathrm{NTf}_{2}$

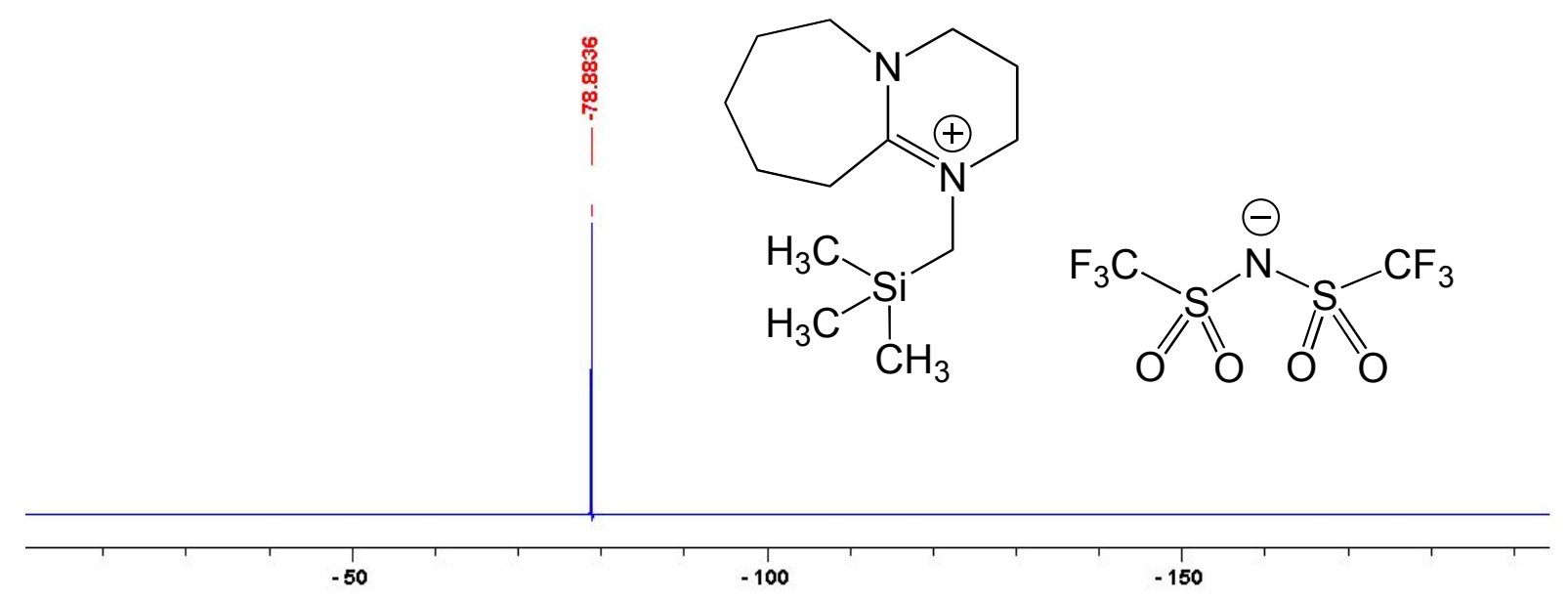

Figure S10. ${ }^{1} \mathrm{H}-\mathrm{NMR}$ spectrum for IL product [BM-M-TMSi-im] $\mathrm{NTf}_{2}$
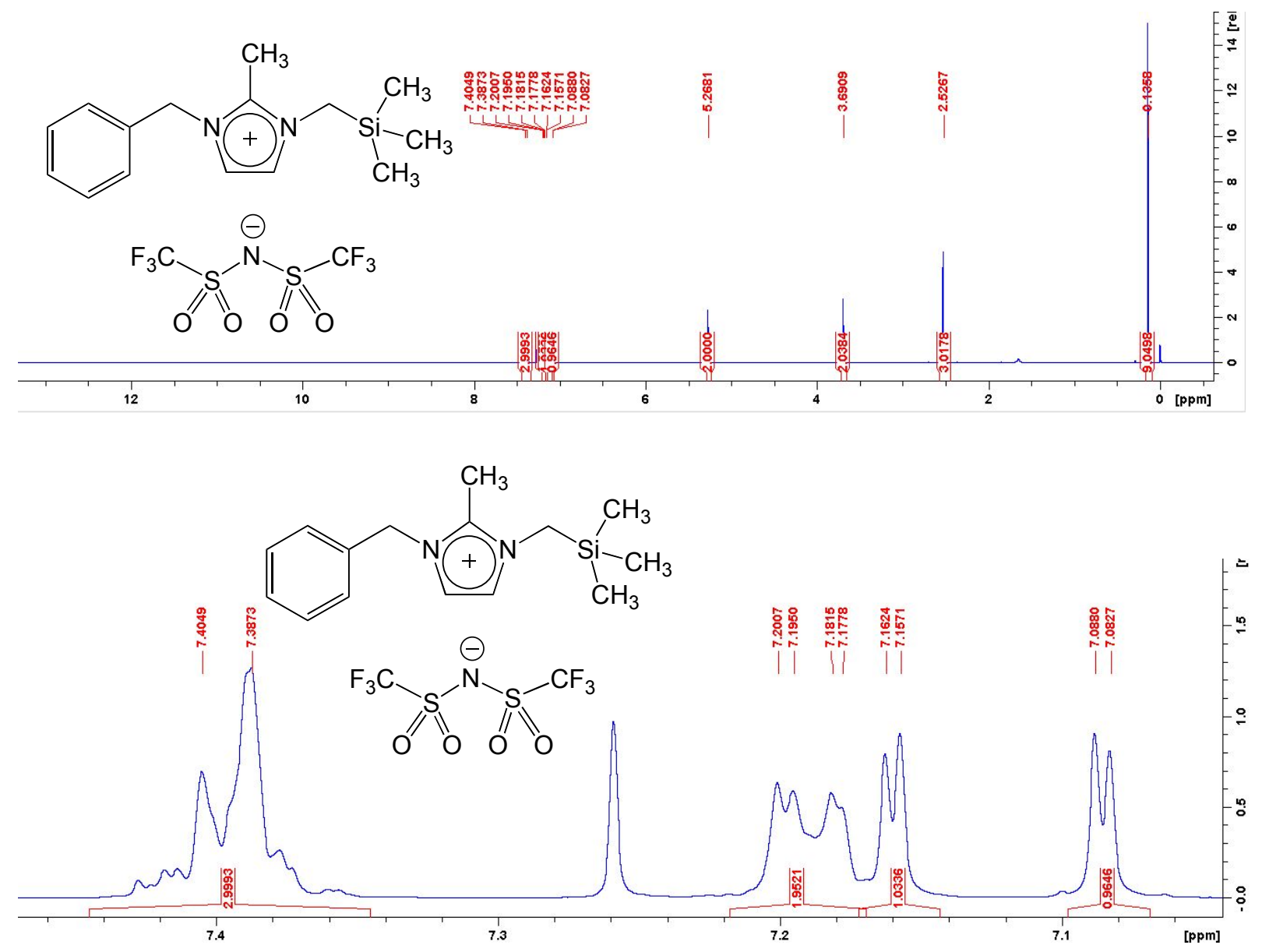
Figure S11. ${ }^{13} \mathrm{C}-\mathrm{NMR}$ spectrum for IL product [BM-M-TMSi-im] NTf $\mathrm{N}_{2}$

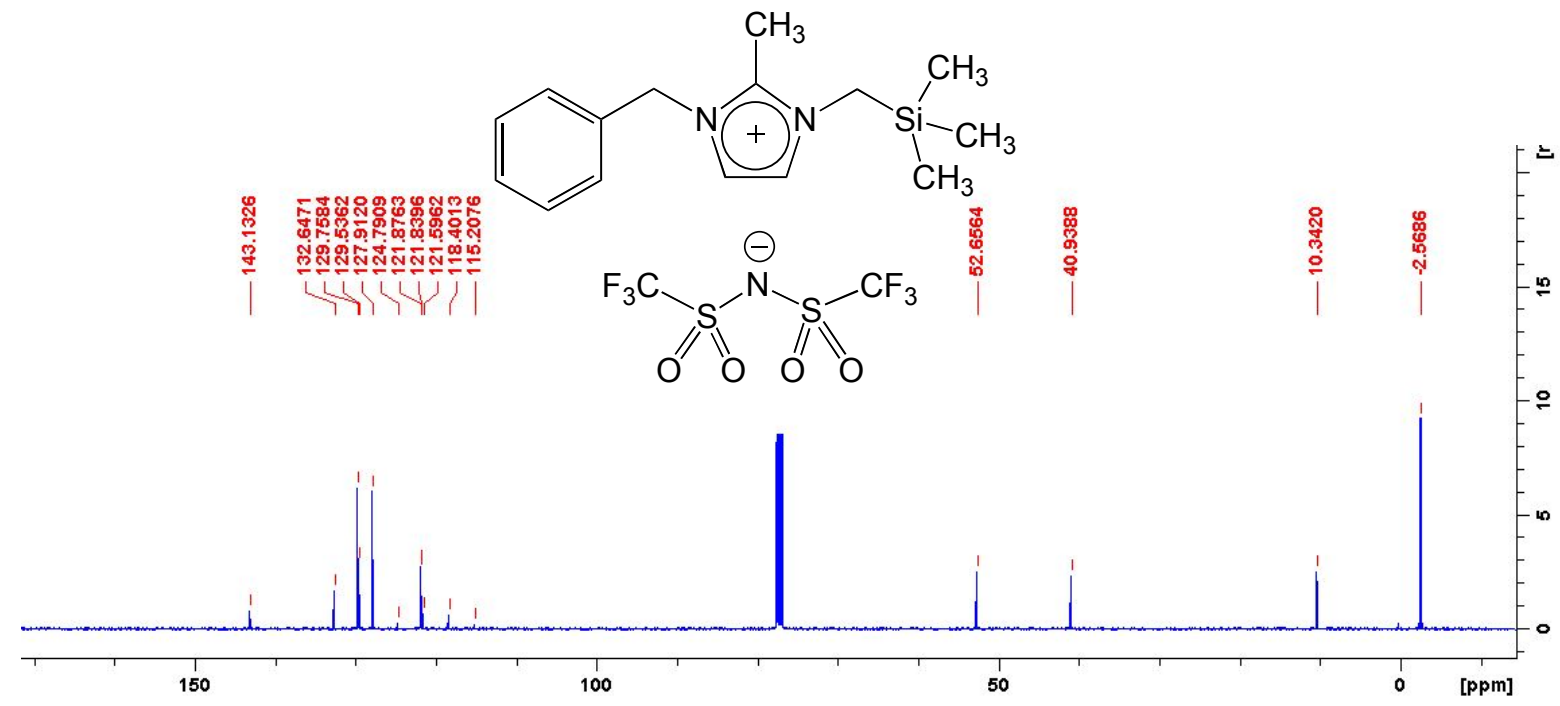

Figure S12. ${ }^{19} \mathrm{~F}-\mathrm{NMR}$ spectrum for IL product [BM-M-TMSi-im] $\mathrm{NTf}_{2}$

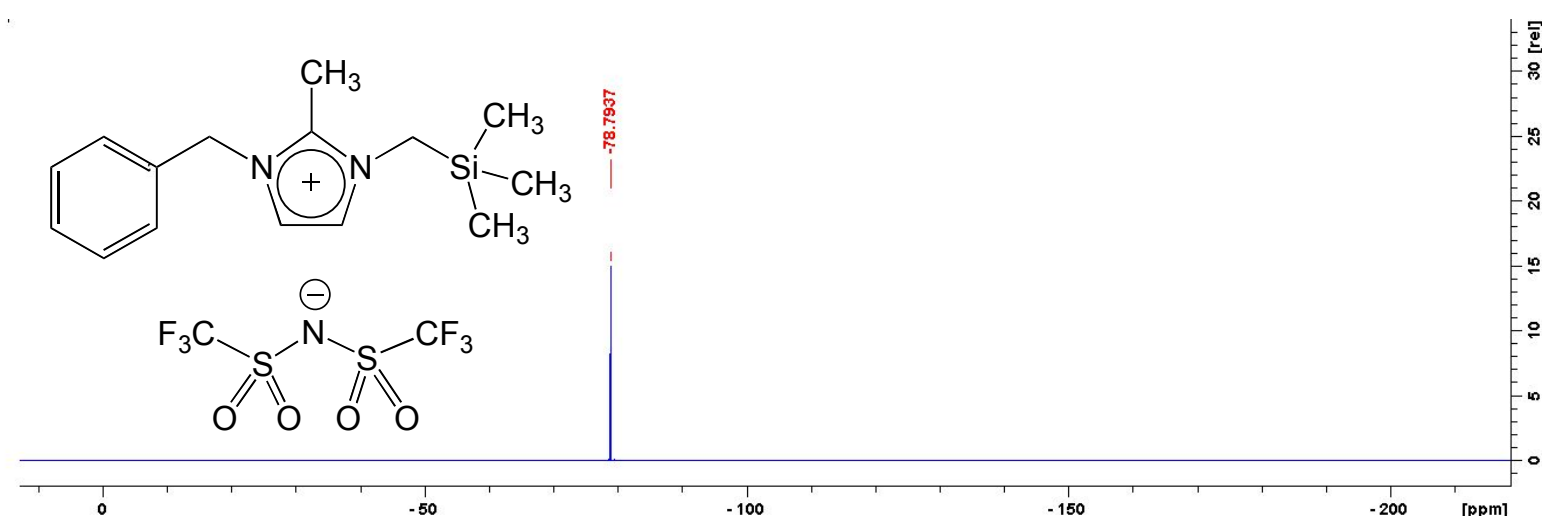

Figure S13. ${ }^{1} \mathrm{H}$-NMR spectrum for starting material 1,2-dimethylimidazole

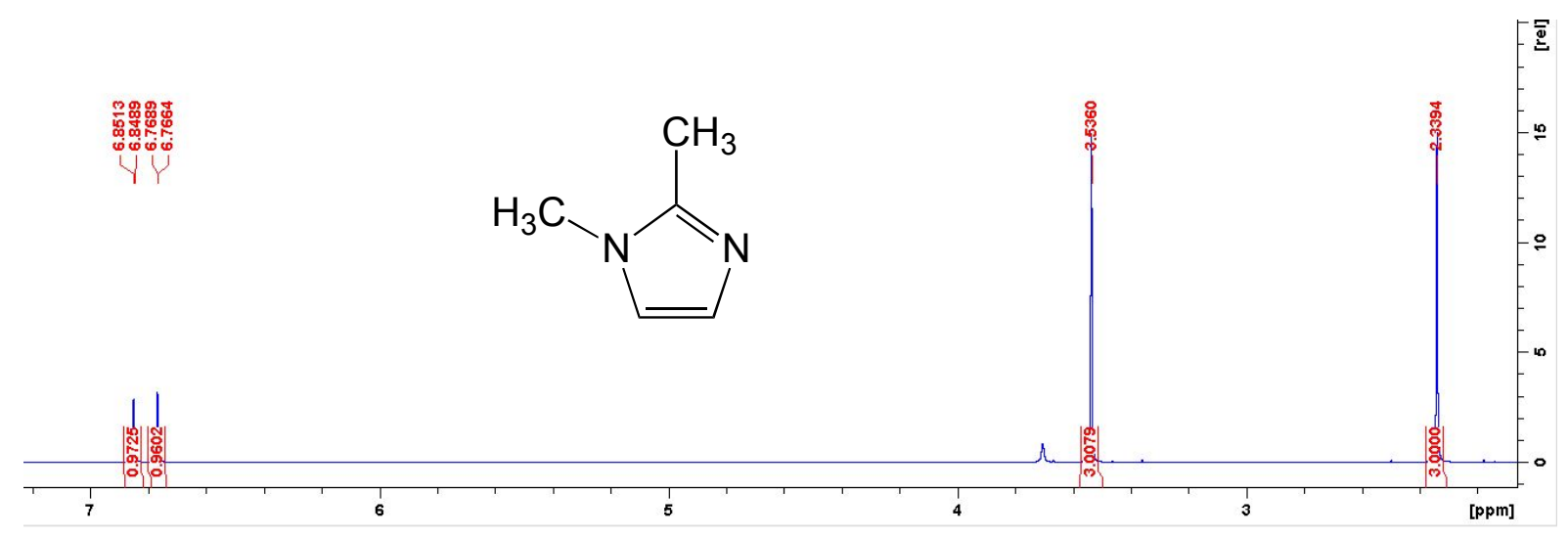


Figure S14. ${ }^{13} \mathrm{C}-\mathrm{NMR}$ spectrum for starting material 1,2-dimethylimidazole

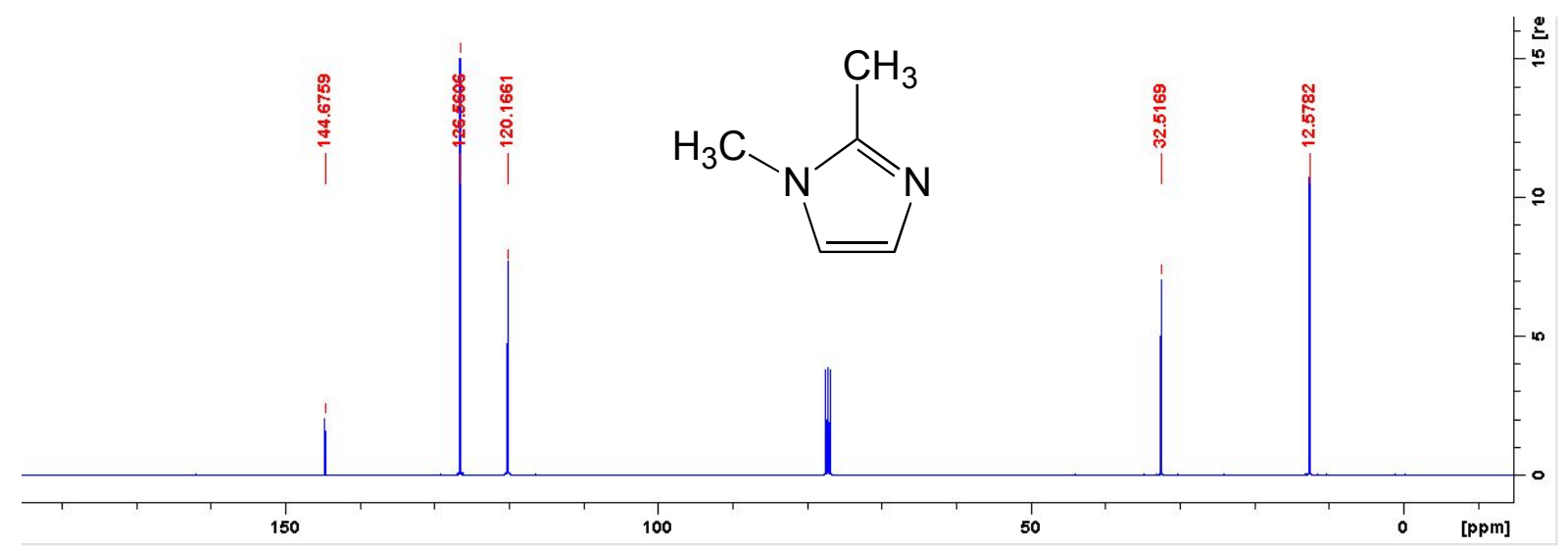

Figure S15. ${ }^{1} \mathrm{H}-\mathrm{NMR}$ spectrum for starting material 1-bromo-3-methylbutane

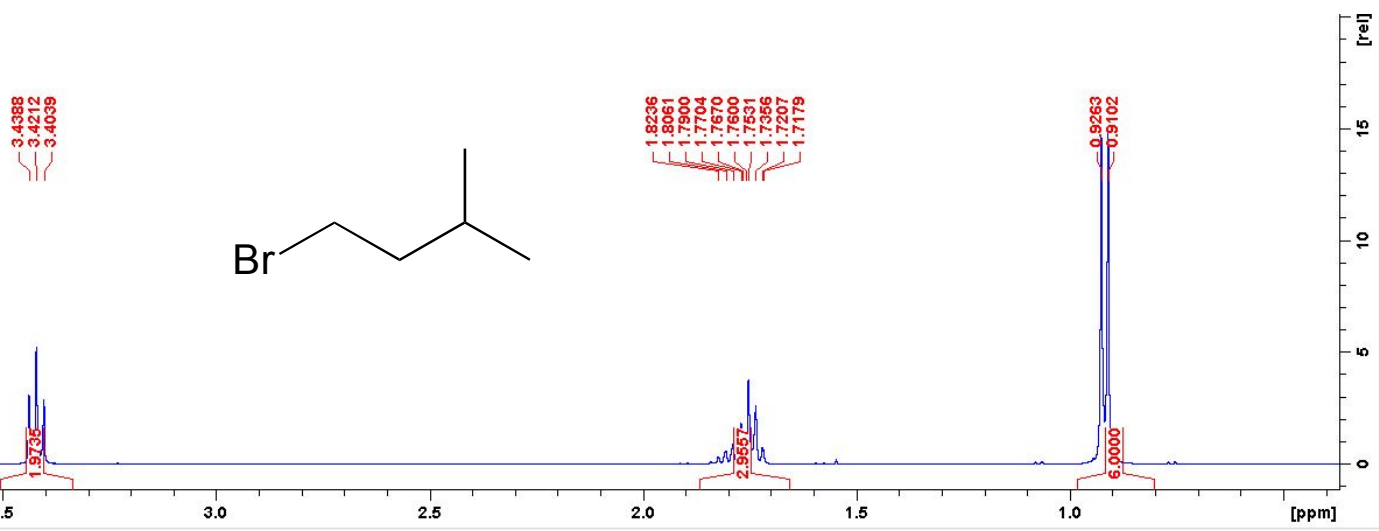

Figure S16. ${ }^{13} \mathrm{C}-\mathrm{NMR}$ spectrum for starting material 1-bromo-3-methylbutane

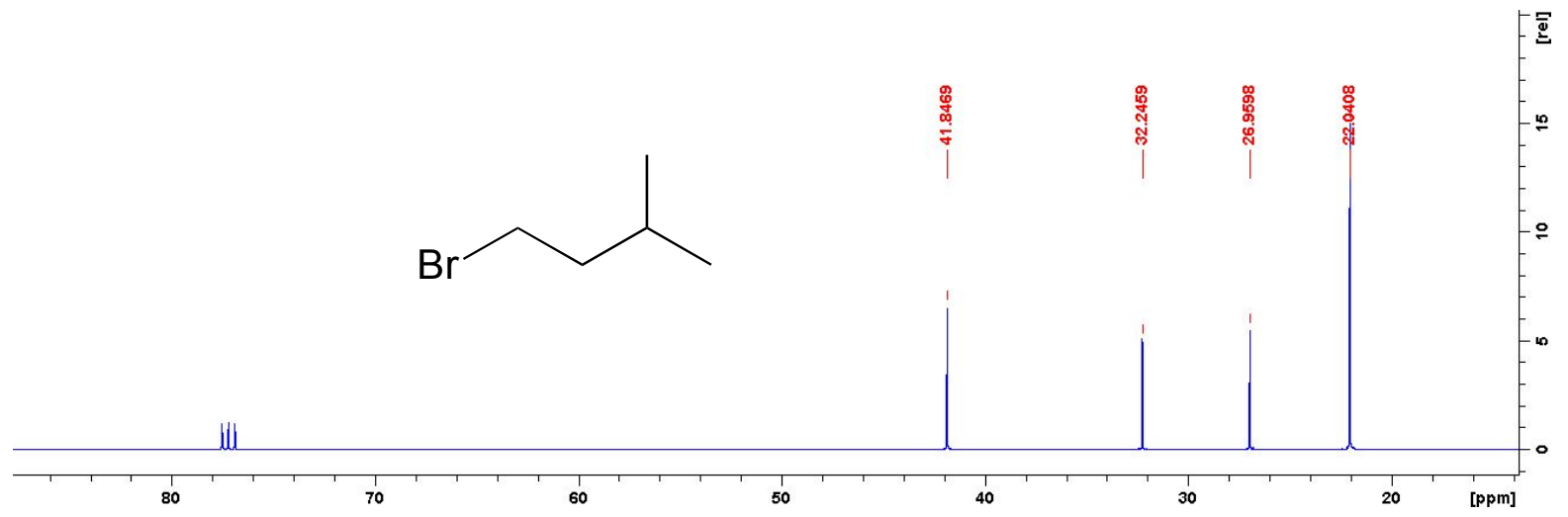


Figure S17. ${ }^{1} \mathrm{H}-\mathrm{NMR}$ spectrum for IL product [DMMB-im] $\mathrm{BF}_{4}$

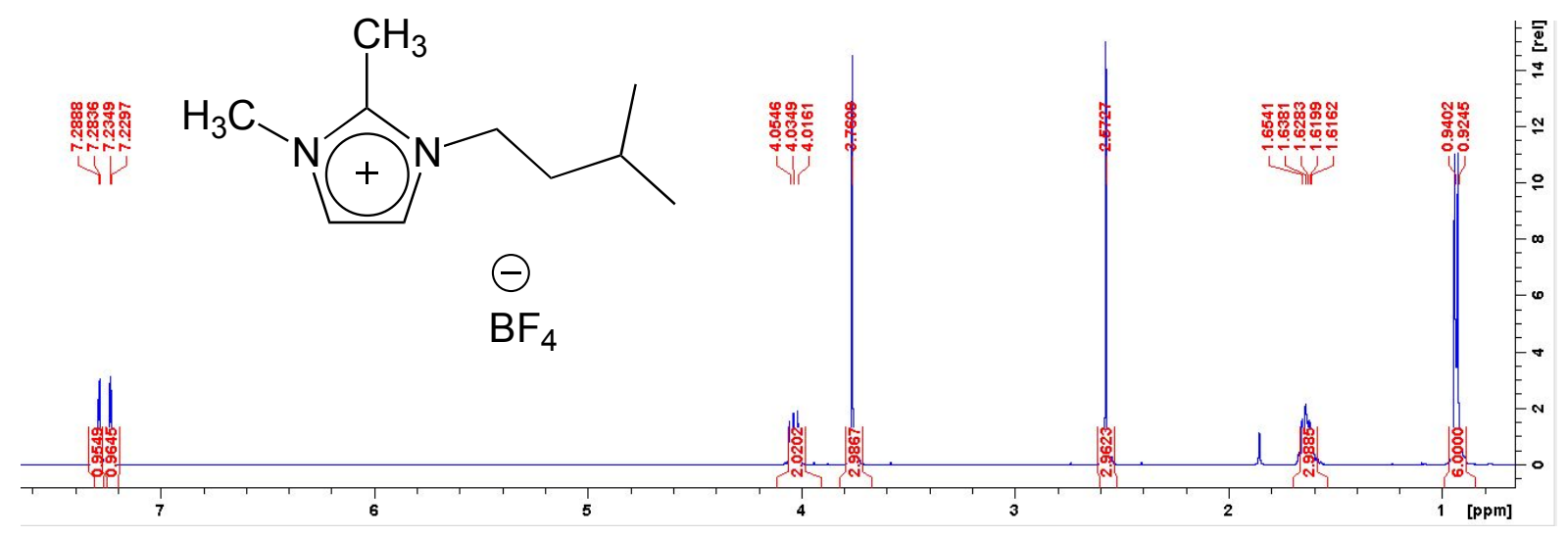

Figure S18. ${ }^{13} \mathrm{C}-\mathrm{NMR}$ spectrum for IL product [DMMB-im] $\mathrm{BF}_{4}$

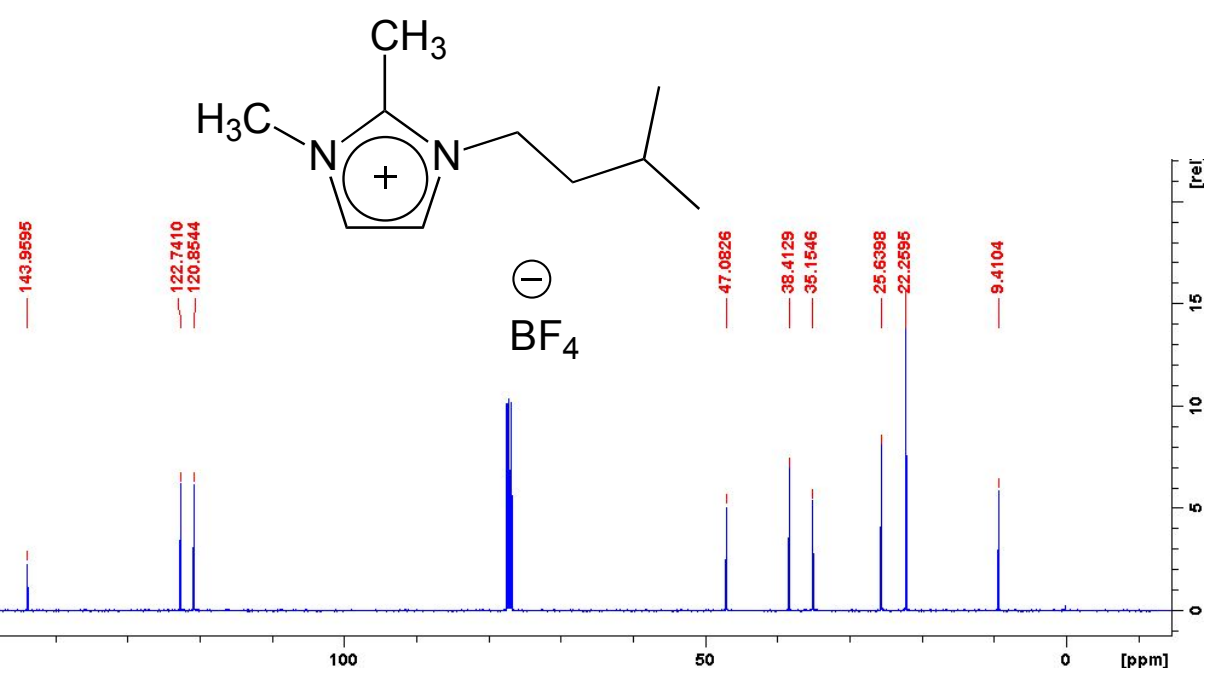

Figure S19. ${ }^{11} \mathrm{~B}-\mathrm{NMR}$ spectrum for IL product [DMMB-im] BF 4

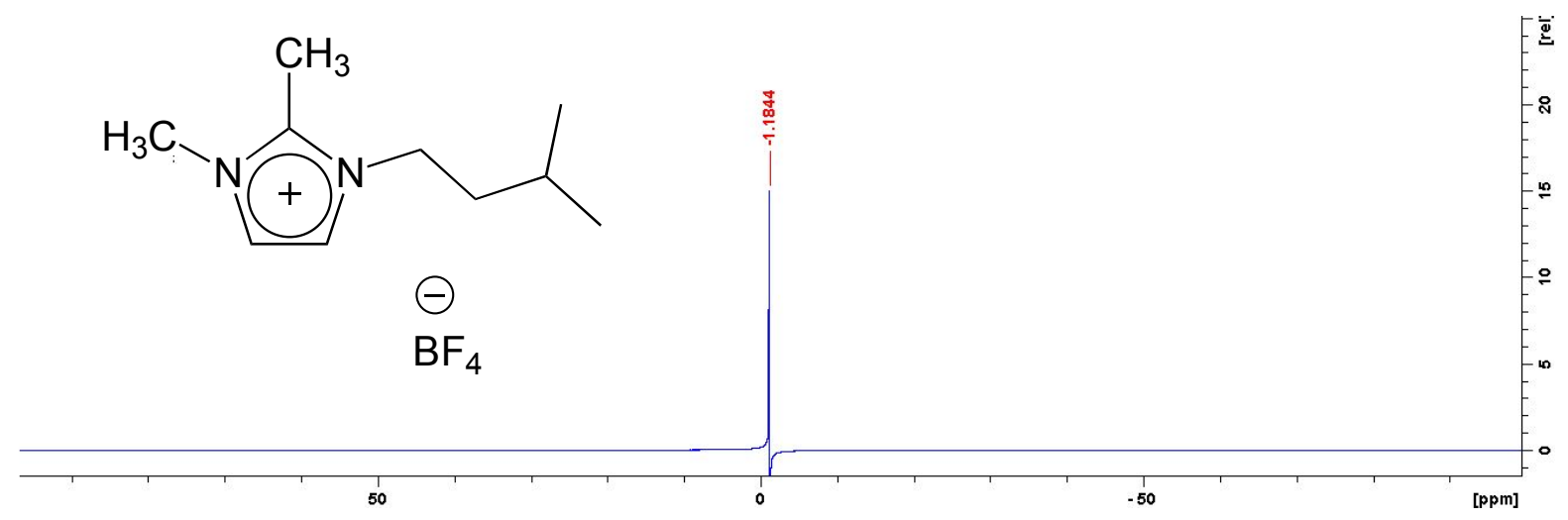


Figure S20. ${ }^{19} \mathrm{~F}-\mathrm{NMR}$ spectrum for IL product [DMMB-im] BF ${ }_{4}$

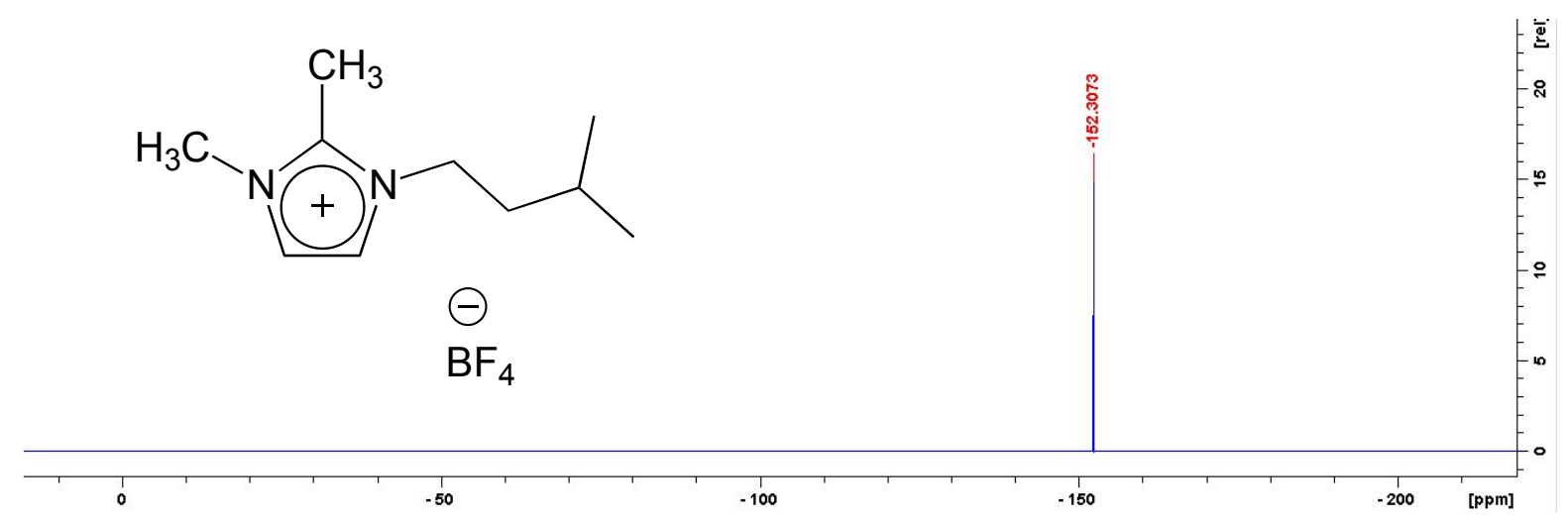

Figure S21. ${ }^{1} \mathrm{H}-\mathrm{NMR}$ spectrum for IL product [DMMB-im] $\mathrm{NTf}_{2}$
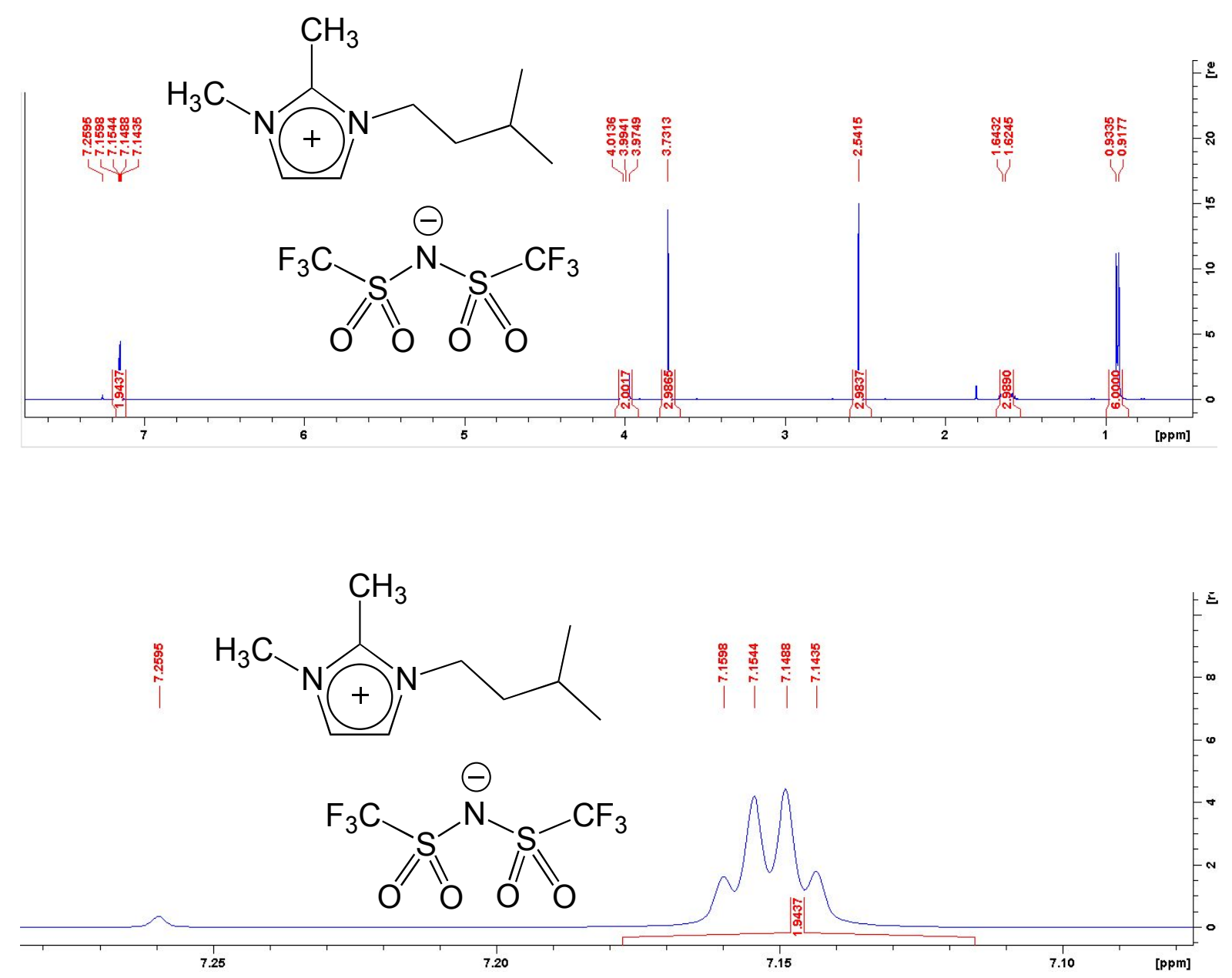


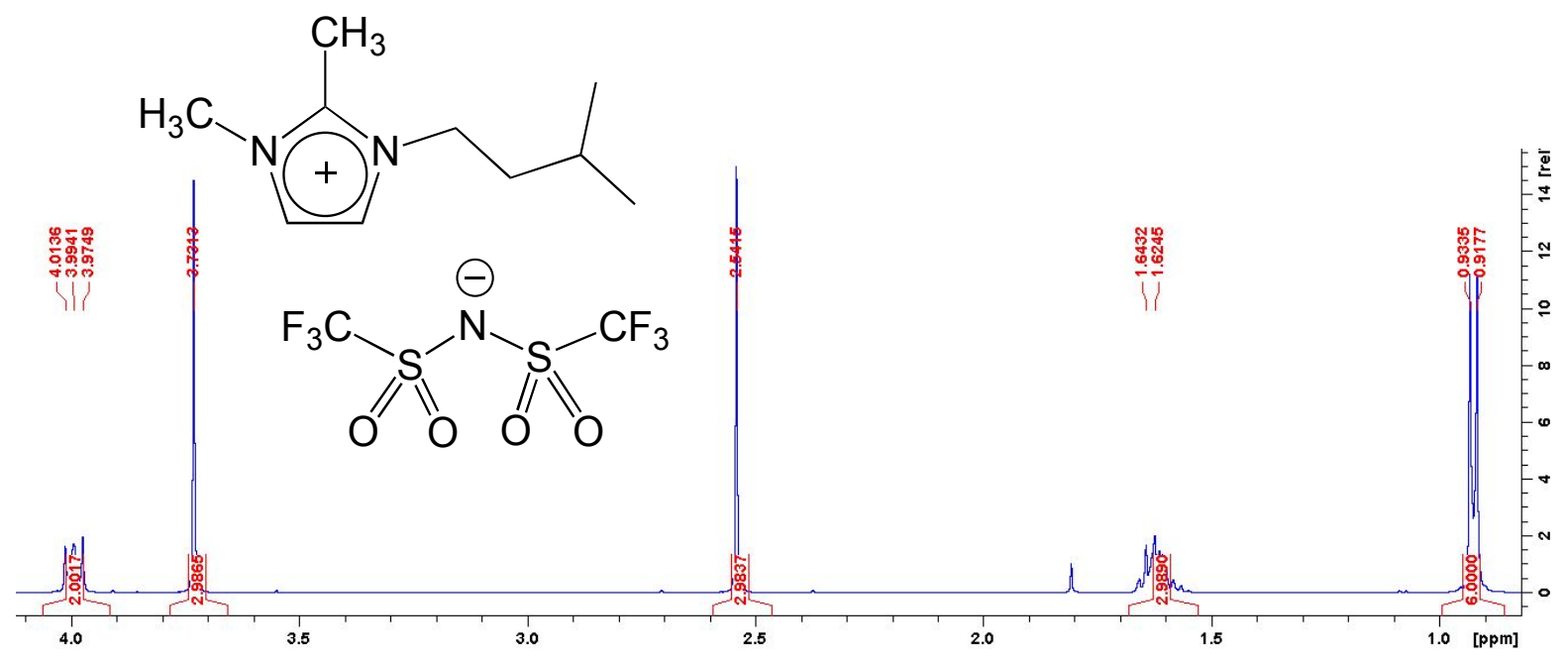

Figure S22. ${ }^{13} \mathrm{C}-\mathrm{NMR}$ spectrum for IL product [DMMB-im] $\mathrm{NTf}_{2}$

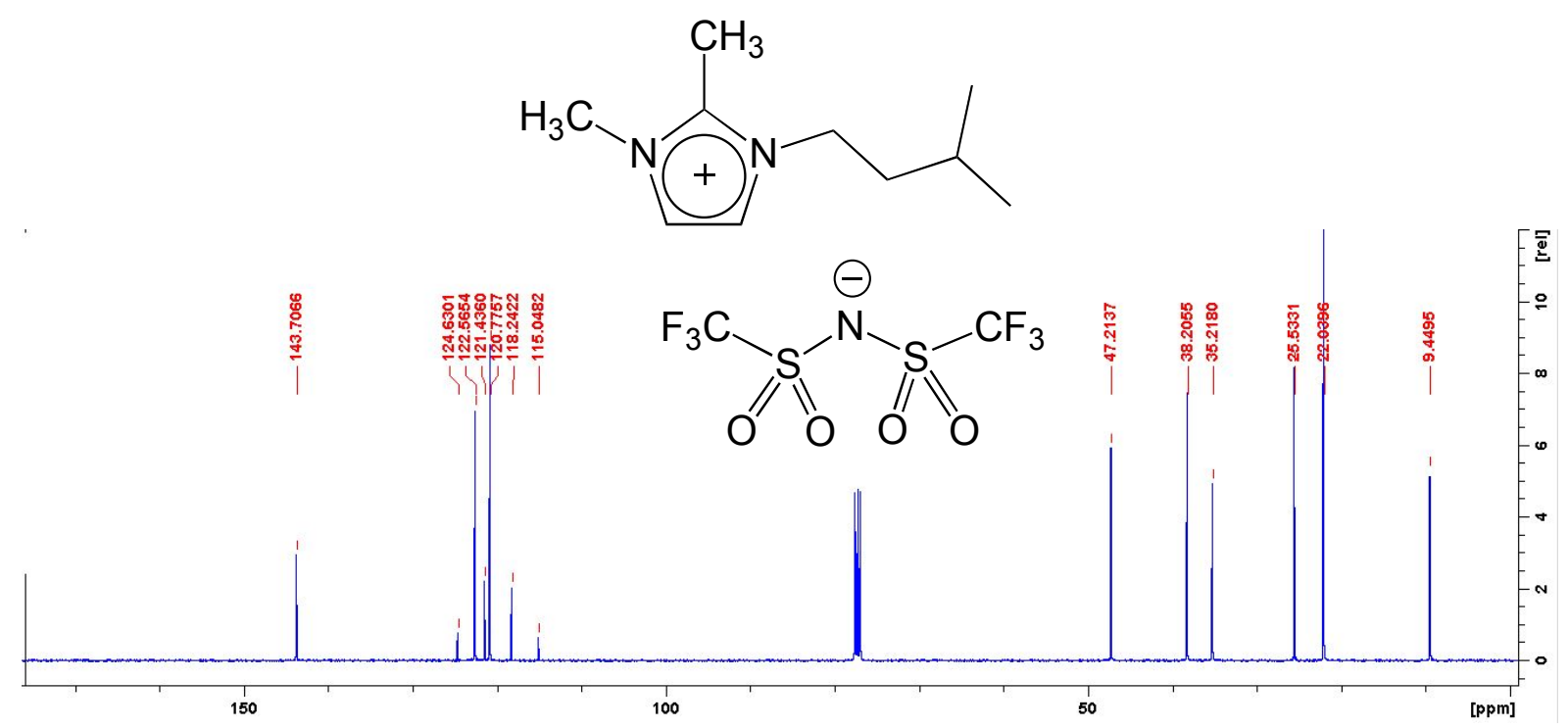

Figure S23. ${ }^{19} \mathrm{~F}-\mathrm{NMR}$ spectrum for IL product [DMMB-im] NTf ${ }_{2}$

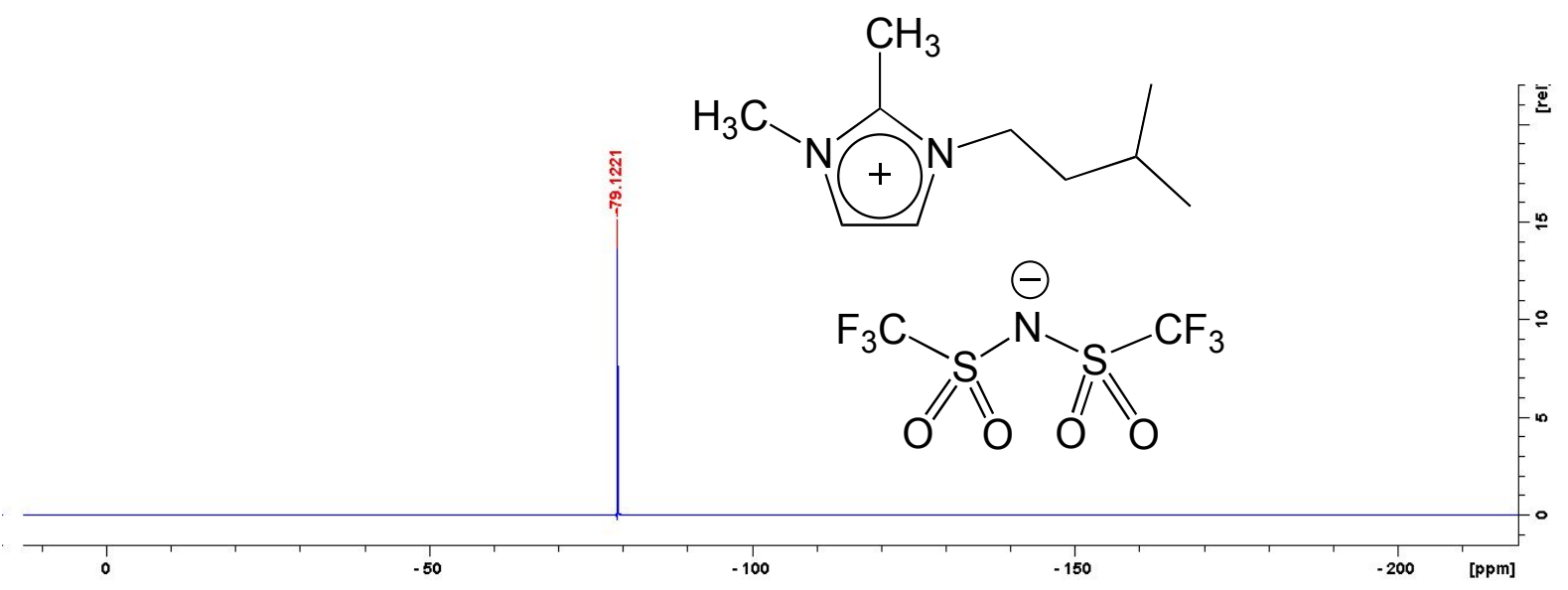


Figure S24. ${ }^{1} \mathrm{H}-\mathrm{NMR}$ spectrum for starting material benzylbromide

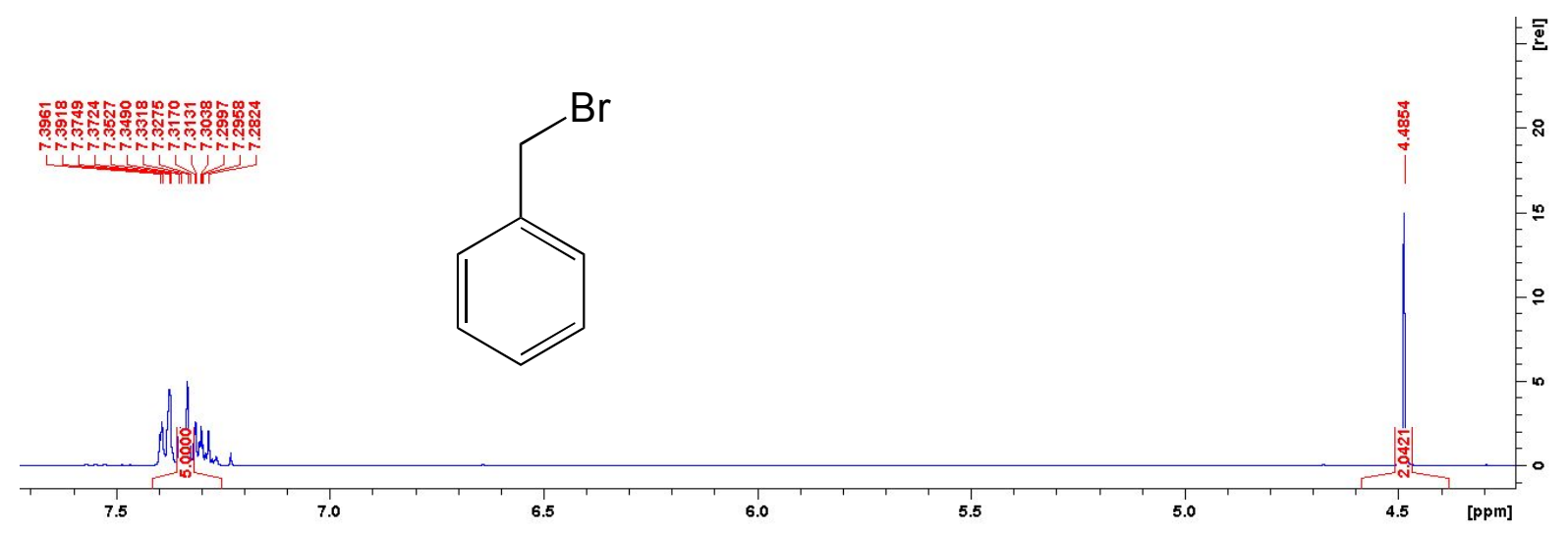

Figure S25. ${ }^{13} \mathrm{C}-\mathrm{NMR}$ spectrum for starting material benzylbromide

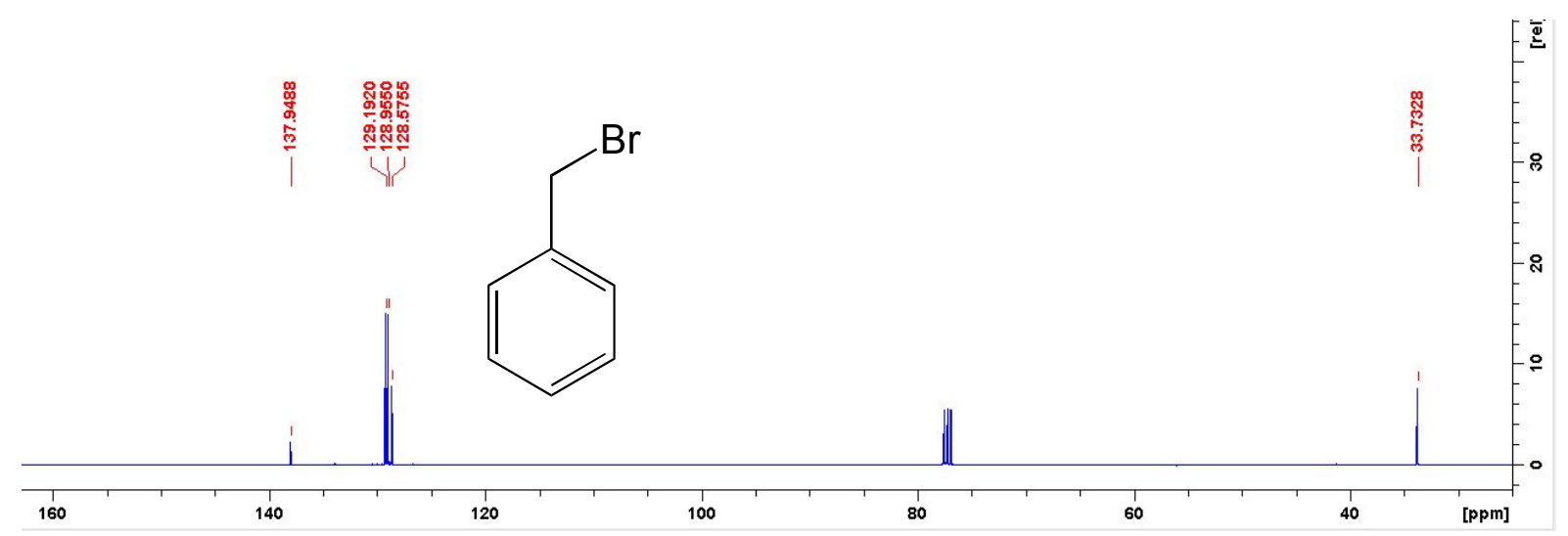

Figure S26. ${ }^{1} \mathrm{H}-\mathrm{NMR}$ spectrum for starting material trioctylphosphine

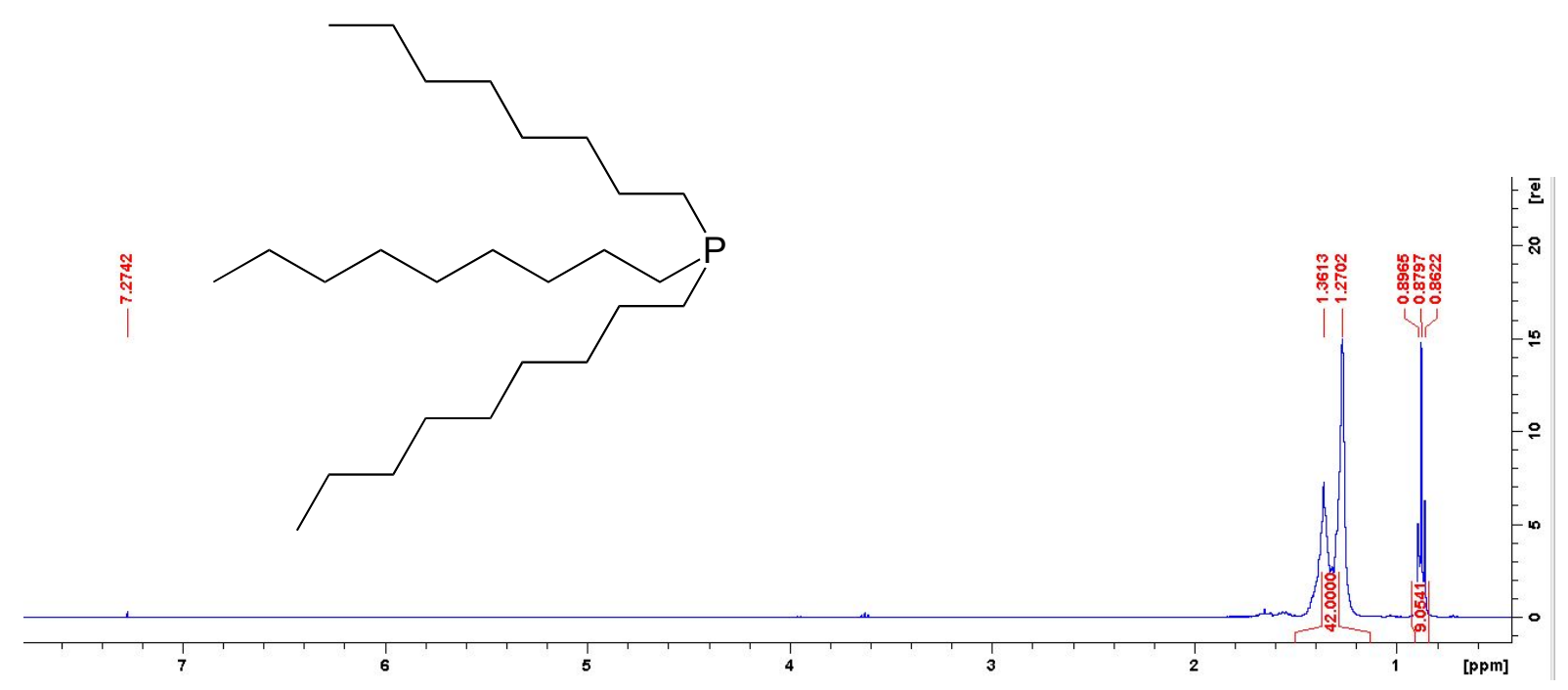


Figure S27. ${ }^{13} \mathrm{C}-\mathrm{NMR}$ spectrum for starting material trioctylphosphine
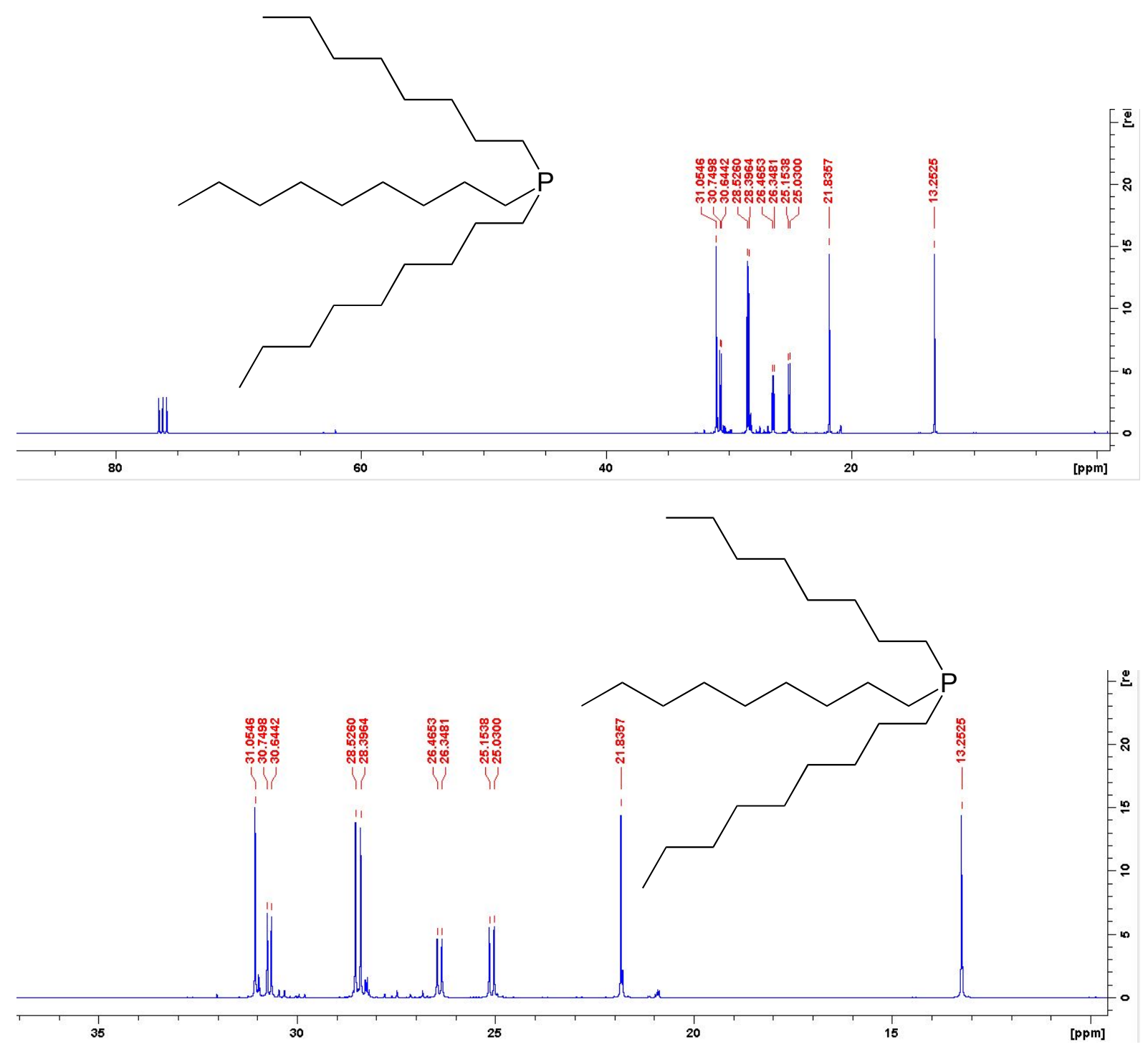

Figure S28. ${ }^{31} \mathrm{P}-\mathrm{NMR}$ spectrum for starting material trioctylphosphine

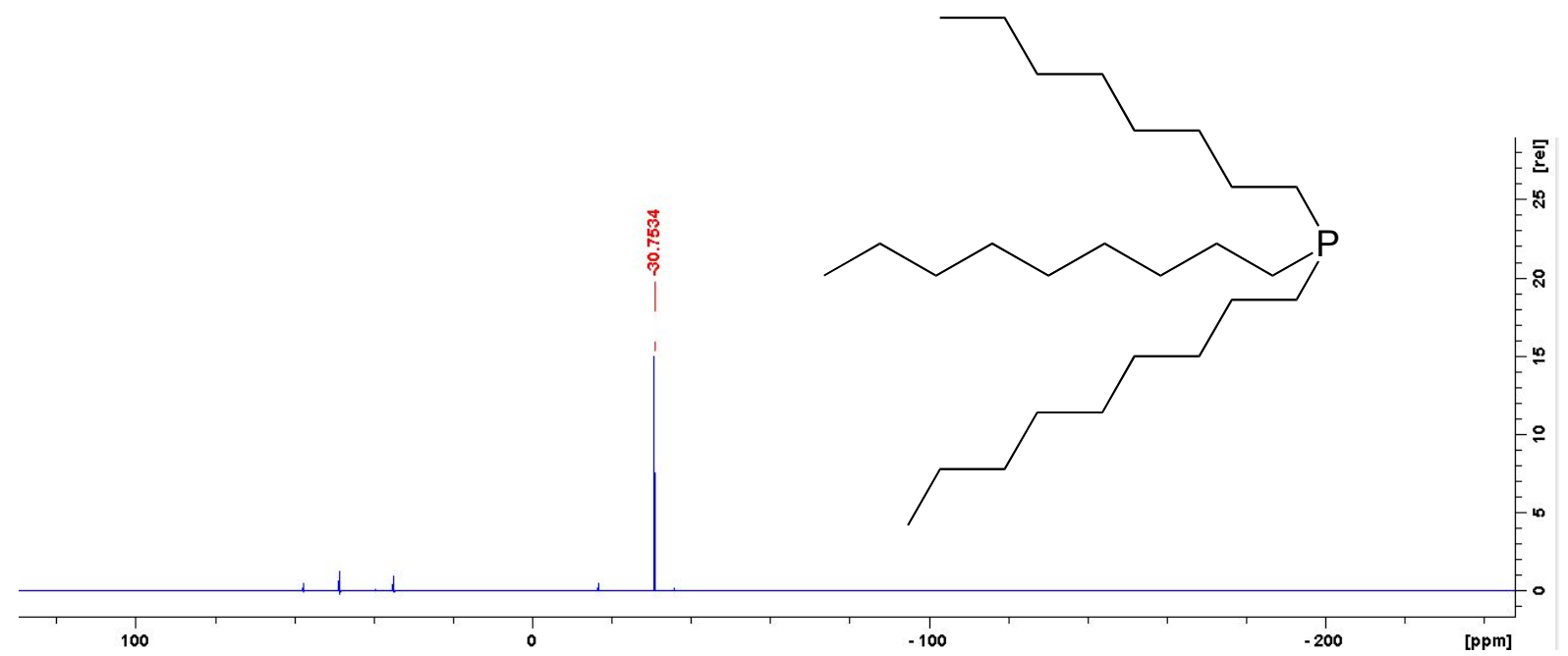


Figure S29. ${ }^{1} \mathrm{H}-\mathrm{NMR}$ spectrum for IL product $\left[\mathrm{BP}_{888}\right] \mathrm{NTf}_{2}$
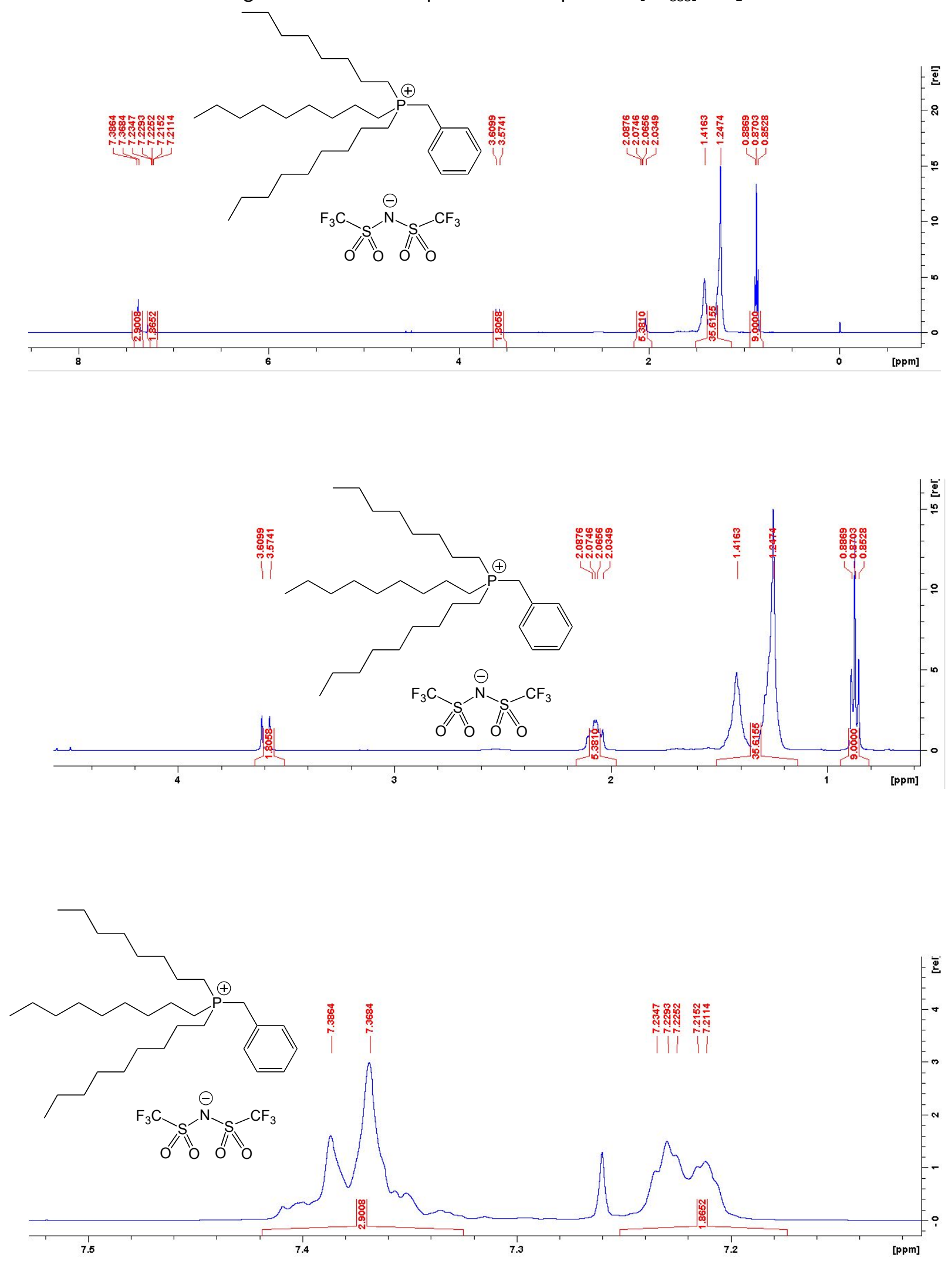
Figure S30. ${ }^{13} \mathrm{C}-\mathrm{NMR}$ spectrum for IL product $\left[\mathrm{BP}_{888}\right] \mathrm{NTf}_{2}$

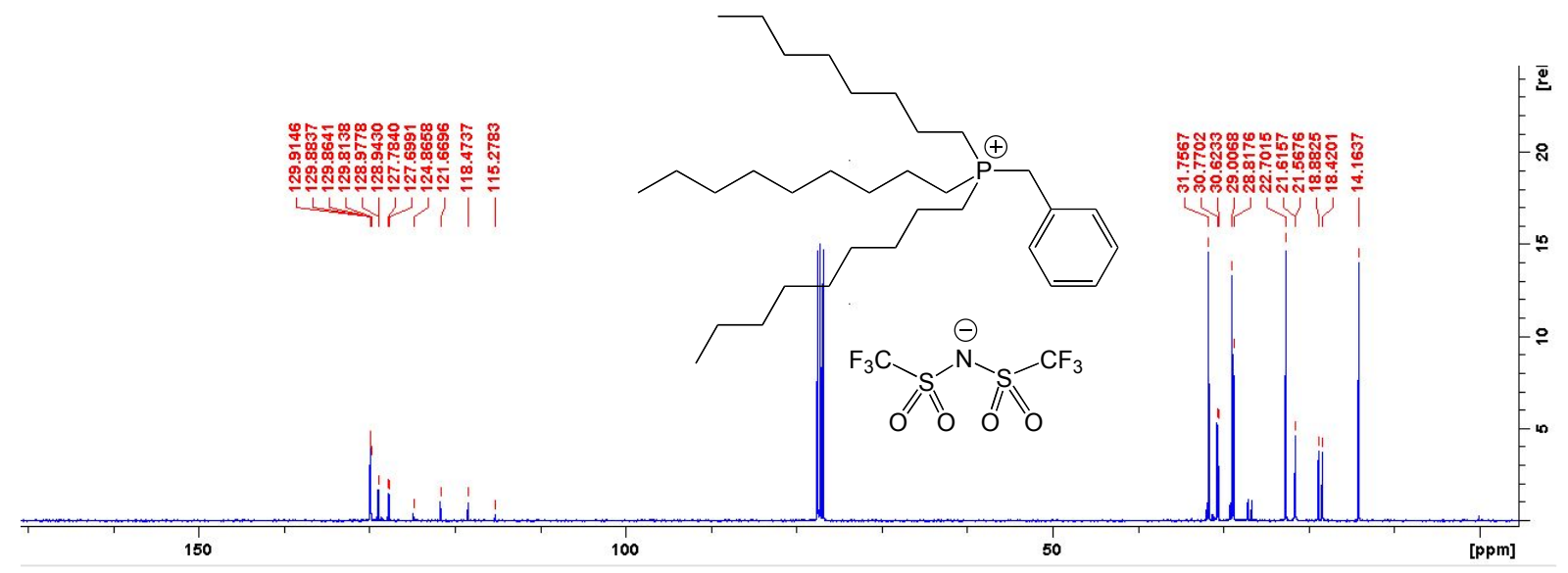

Figure S31. ${ }^{19} \mathrm{~F}-\mathrm{NMR}$ spectrum for IL product $\left[\mathrm{BP}_{888}\right] \mathrm{NTf}_{2}$

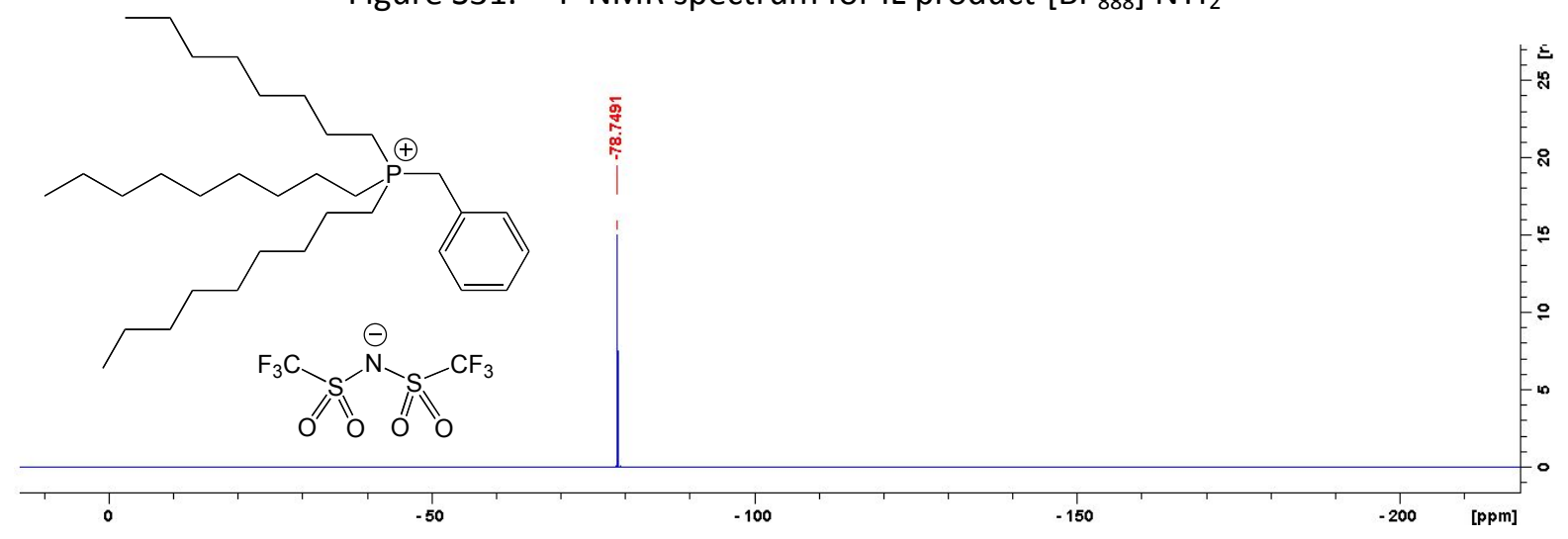

Figure S32. ${ }^{31} \mathrm{P}-\mathrm{NMR}$ spectrum for IL product $\left[\mathrm{BP}_{888}\right] \mathrm{NTf}_{2}$

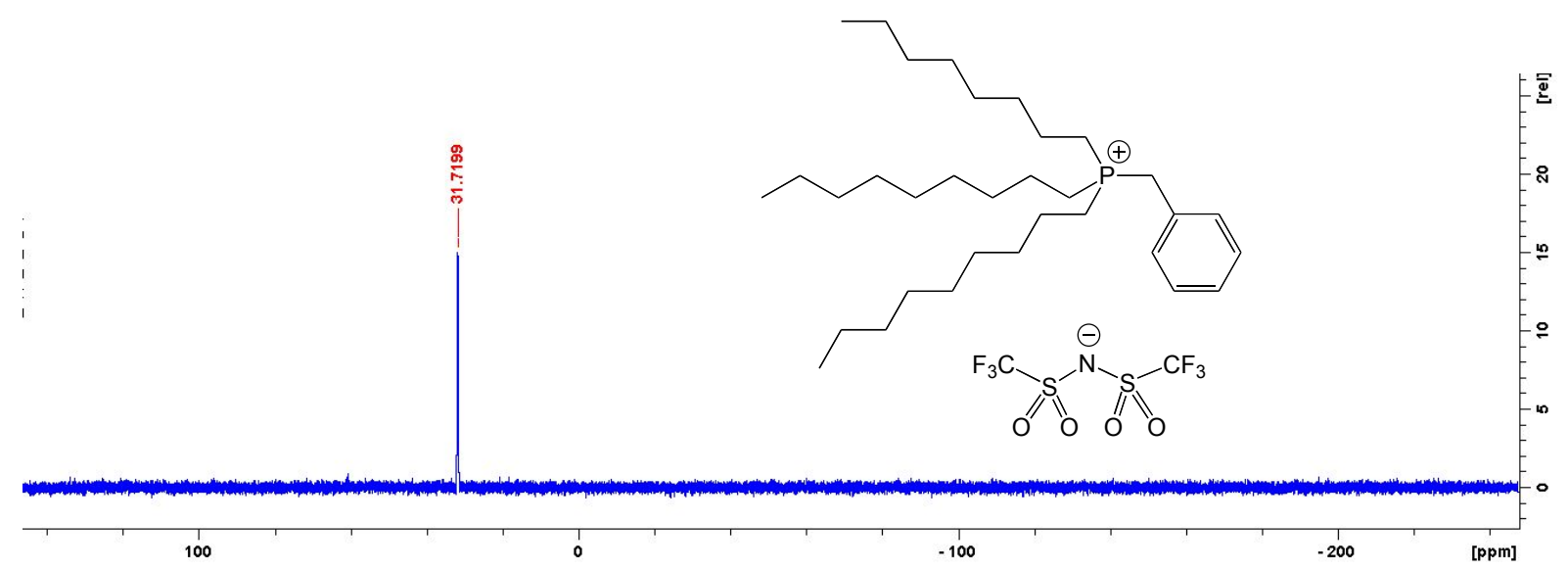

Figure S33. ${ }^{1} \mathrm{H}-\mathrm{NMR}$ spectrum for starting material allyl bromide 

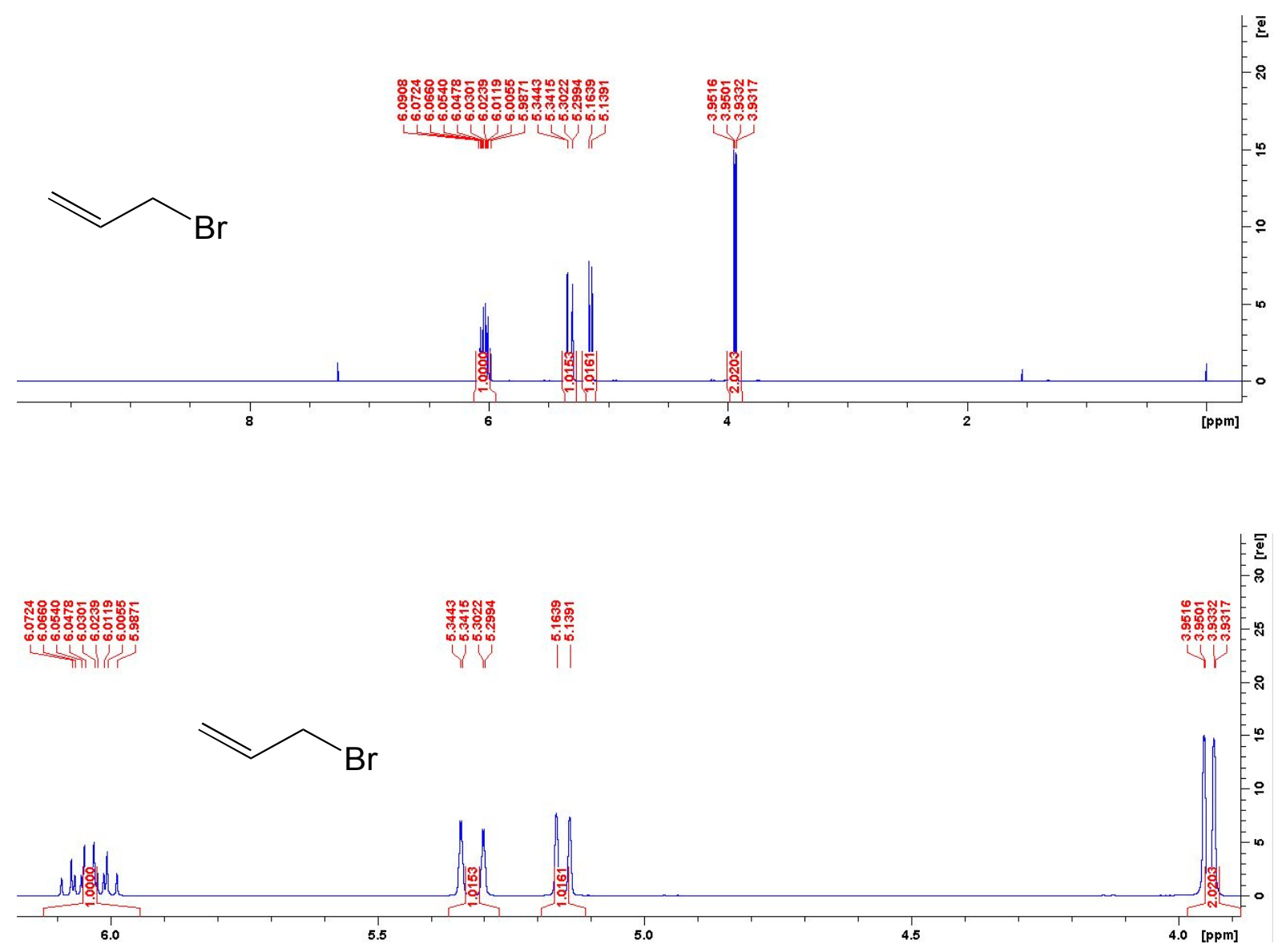

Figure S34. ${ }^{13} \mathrm{C}-\mathrm{NMR}$ spectrum for starting material allyl bromide

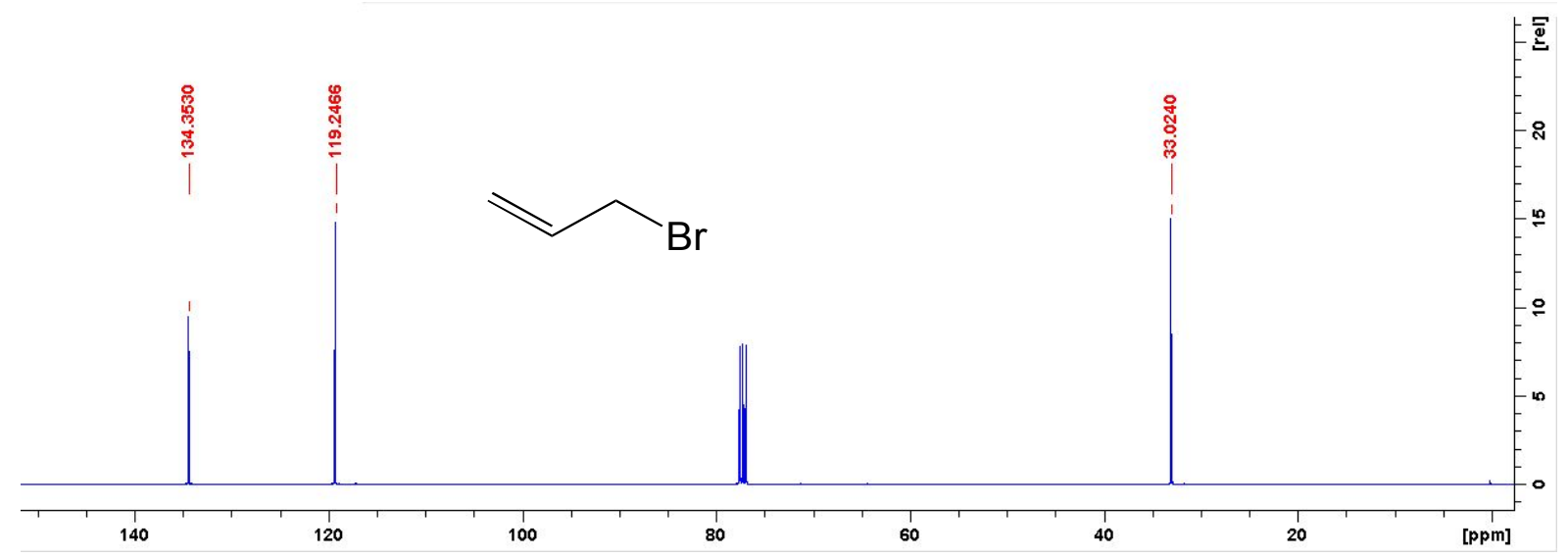


Figure S35. ${ }^{1} \mathrm{H}-\mathrm{NMR}$ spectrum for IL product [ABM-im] $\mathrm{NTf}_{2}$
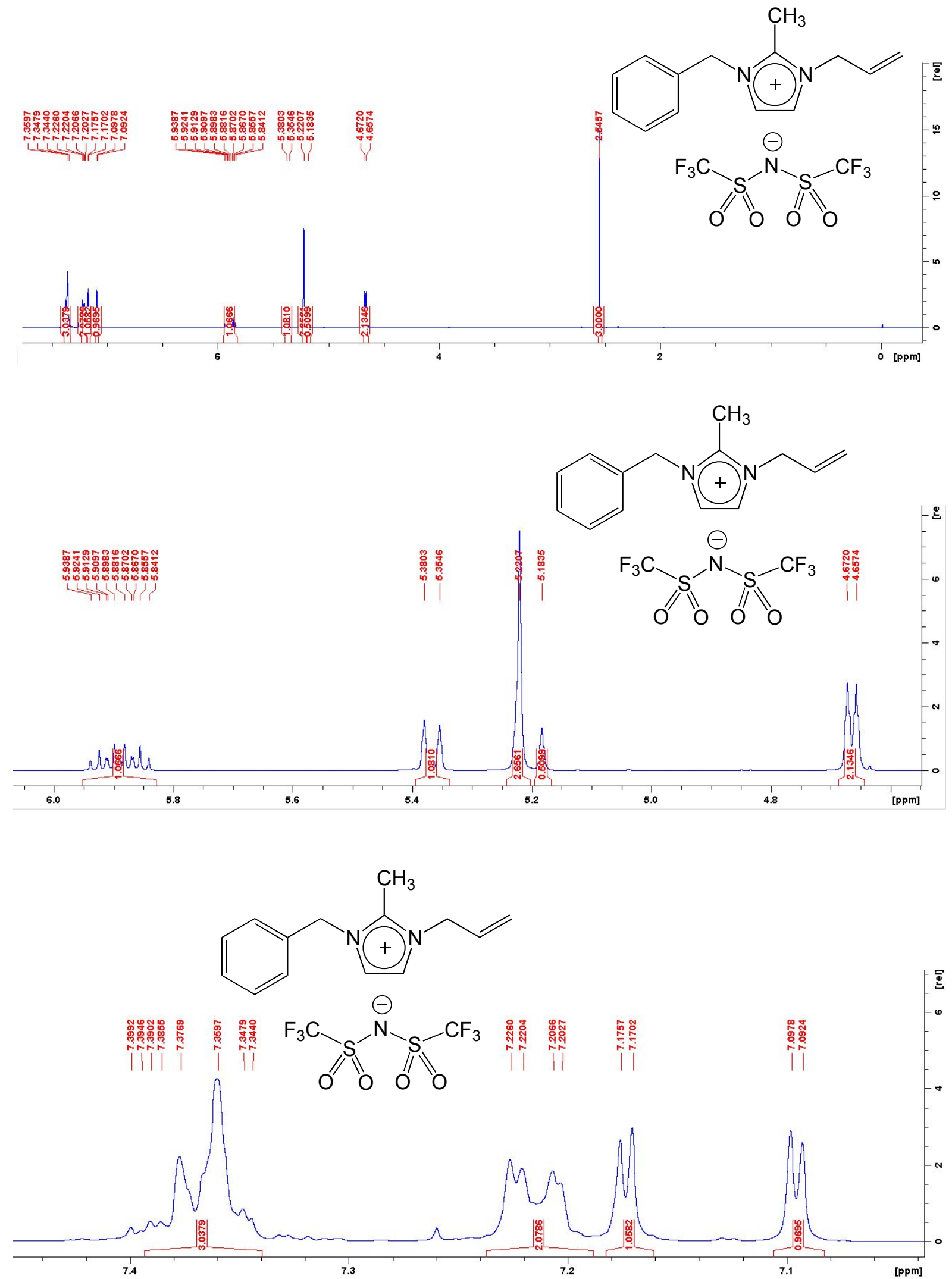
Figure S36. ${ }^{13} \mathrm{C}-\mathrm{NMR}$ spectrum for IL product [ABM-im] $\mathrm{NTf}_{2}$

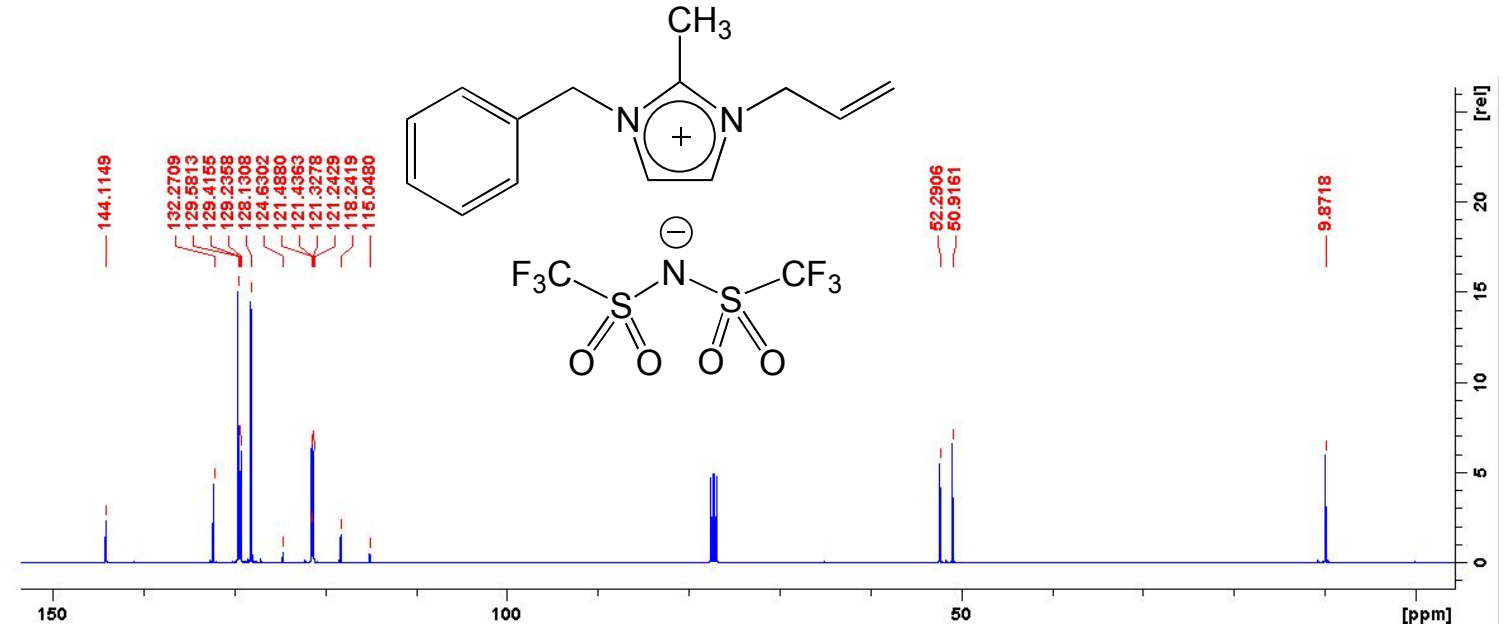

Figure S37. ${ }^{19} \mathrm{~F}-\mathrm{NMR}$ spectrum for IL product [ABM-im] $\mathrm{NTf}_{2}$

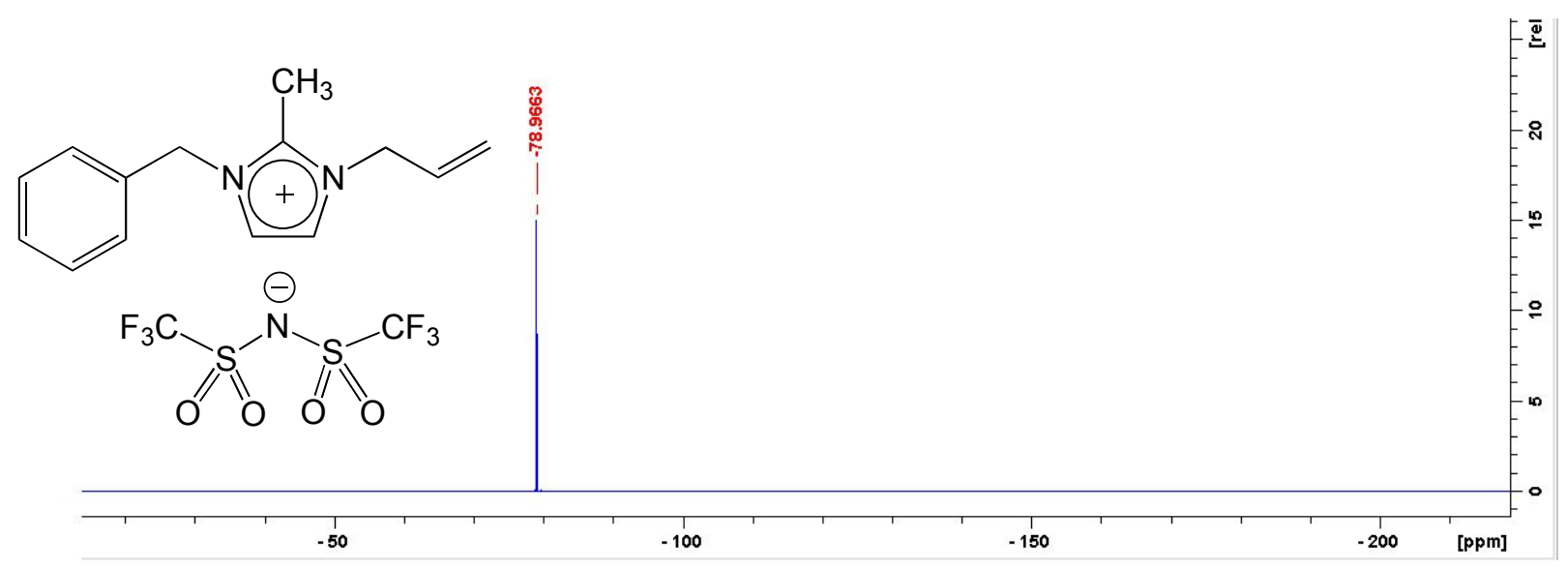

Figure S38. ${ }^{1} \mathrm{H}$-NMR spectrum for starting material 1-benzyl-2-methylimidazole

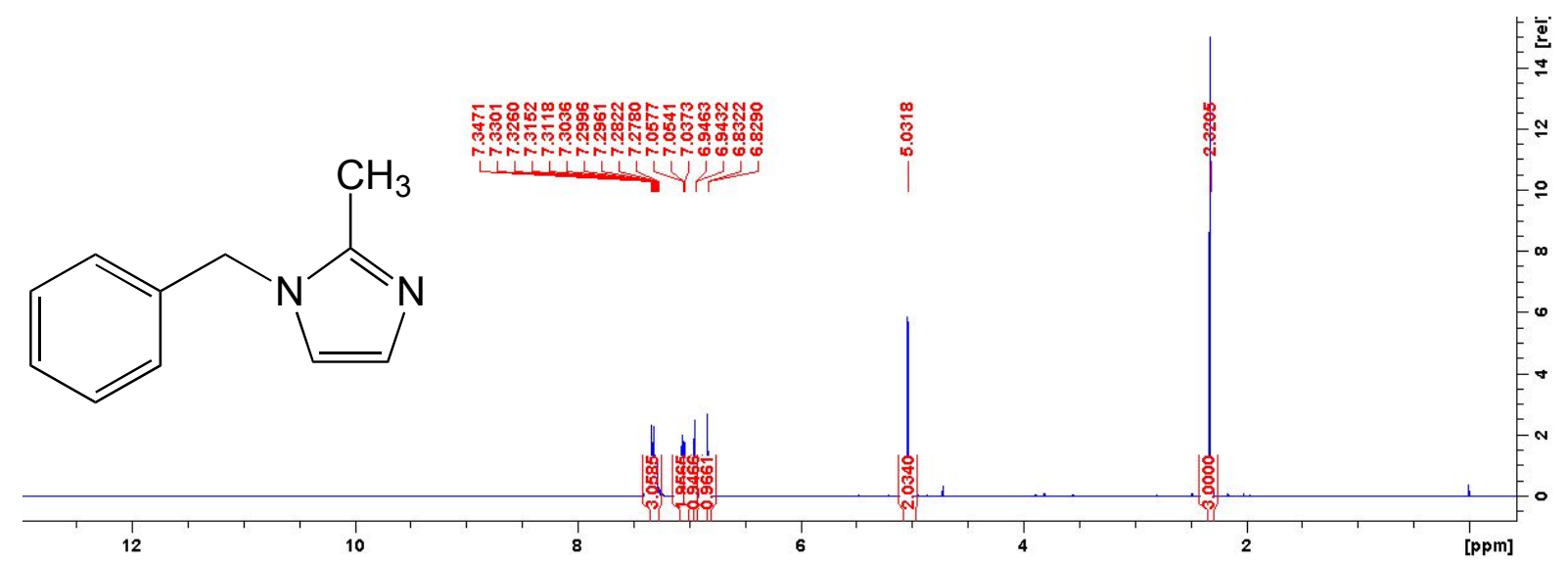




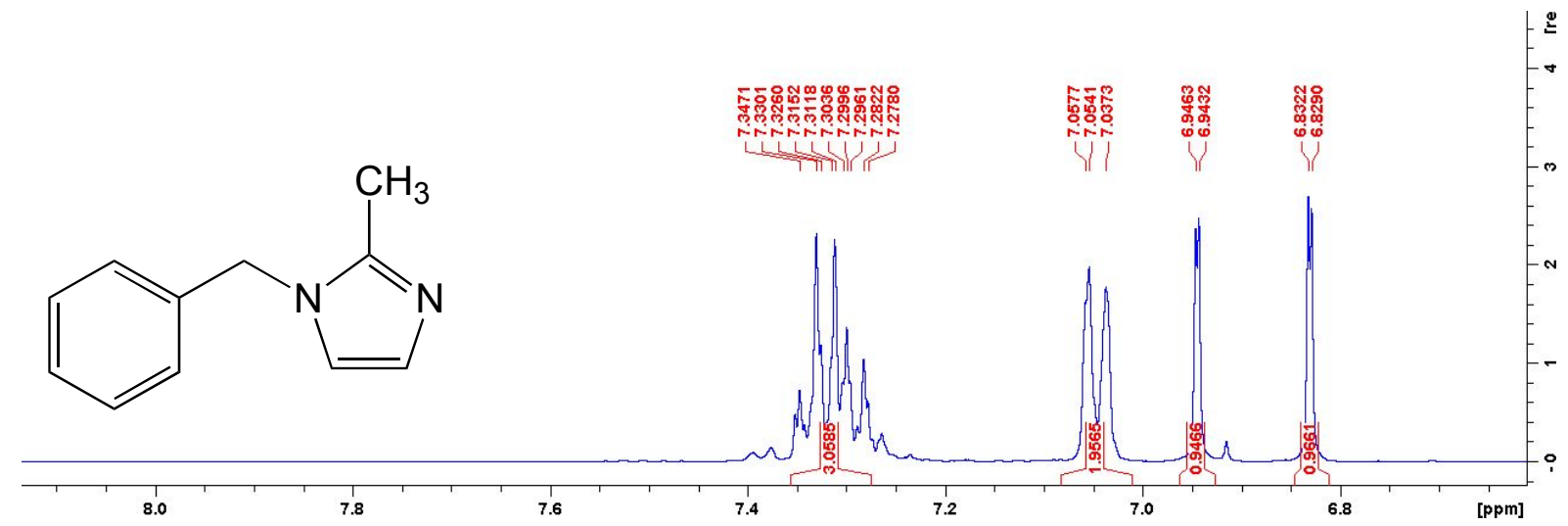

Figure S39. ${ }^{13} \mathrm{C}-\mathrm{NMR}$ spectrum for starting material 1-benzyl-2-methylimidazole

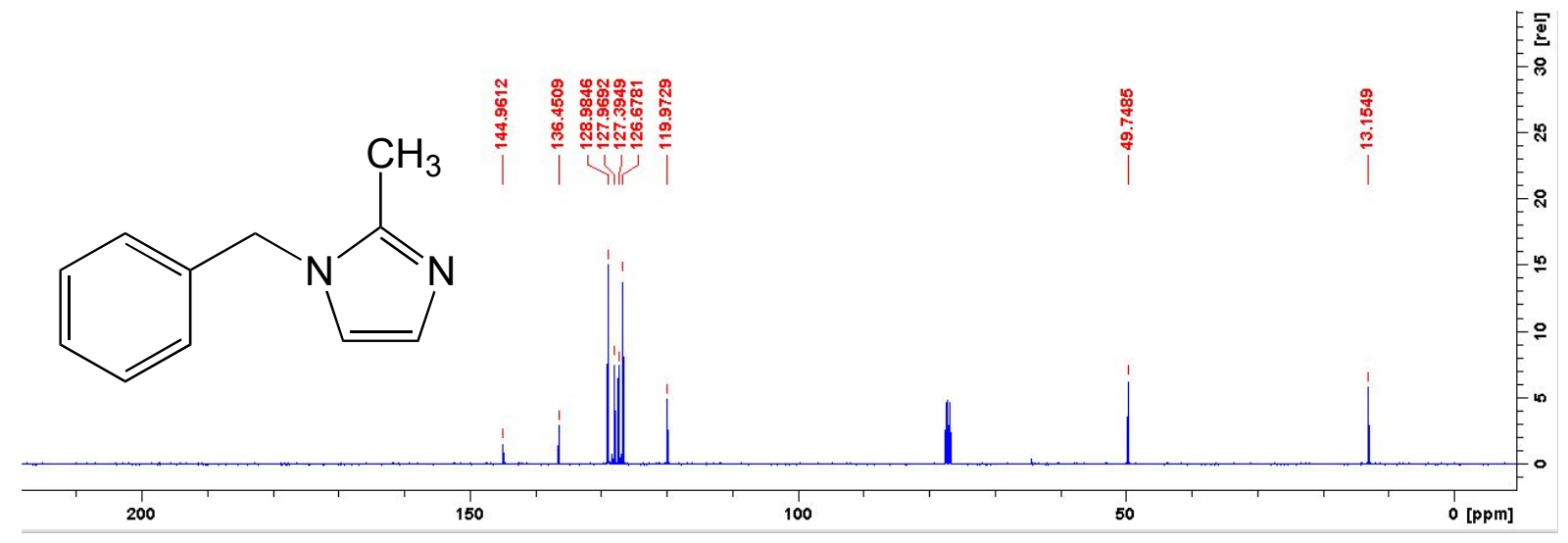

Figure S40. ${ }^{1} \mathrm{H}-\mathrm{NMR}$ spectrum for starting material 1-bromooctane

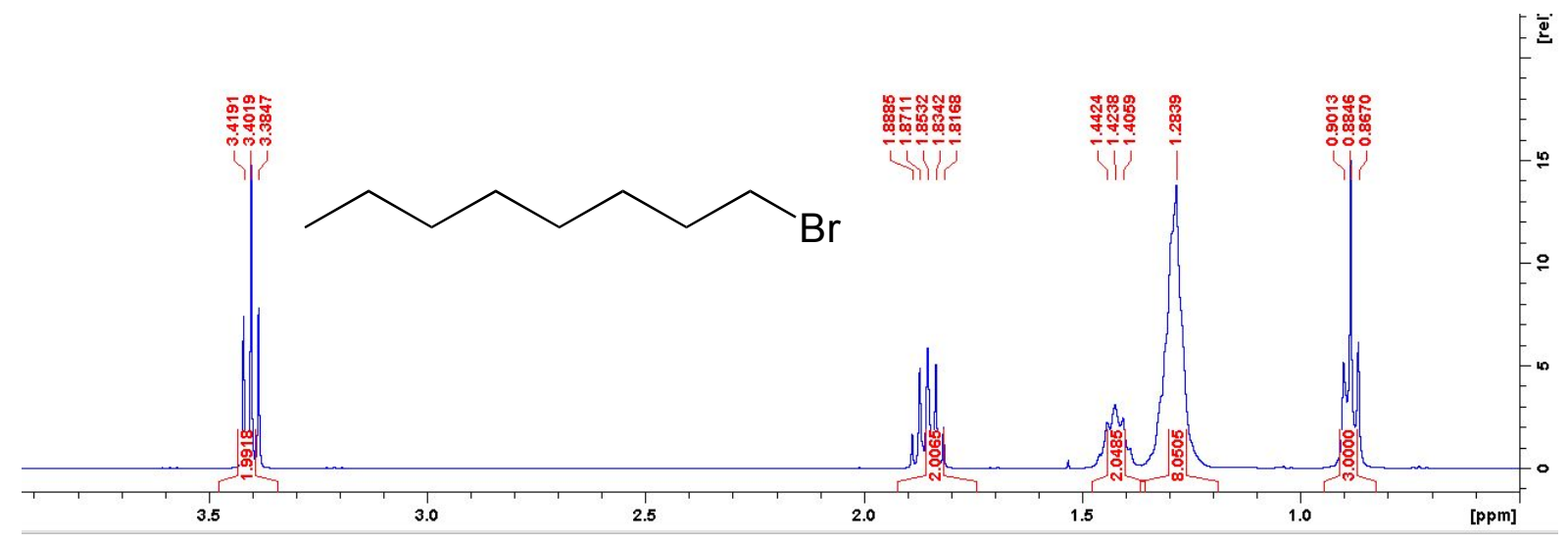


Figure S41. ${ }^{13}$ C-NMR spectrum for starting material 1-bromooctane

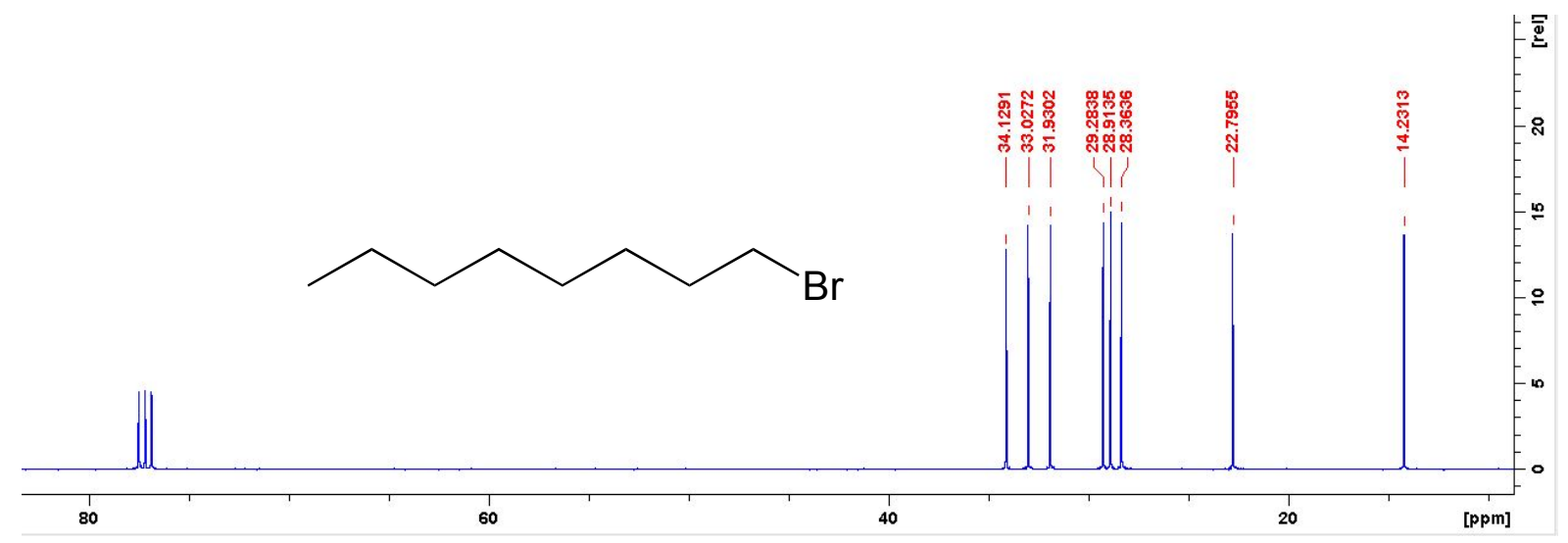

Figure S42. ${ }^{1} \mathrm{H}-\mathrm{NMR}$ spectrum for IL product [BMO-im] $\mathrm{BF}_{4}$

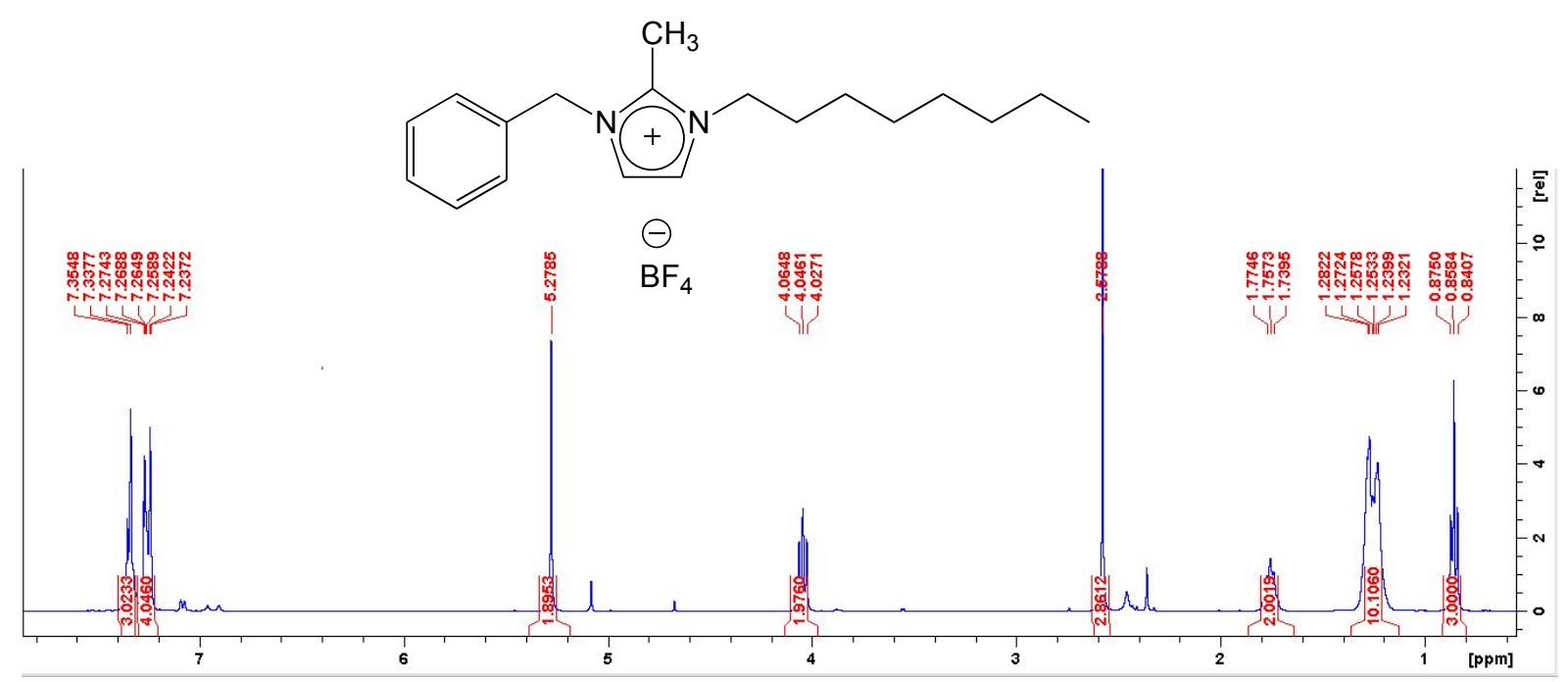

Figure $\mathrm{S} 43 .{ }^{13} \mathrm{C}-\mathrm{NMR}$ spectrum for IL product [BMO-im] $\mathrm{BF}_{4}$

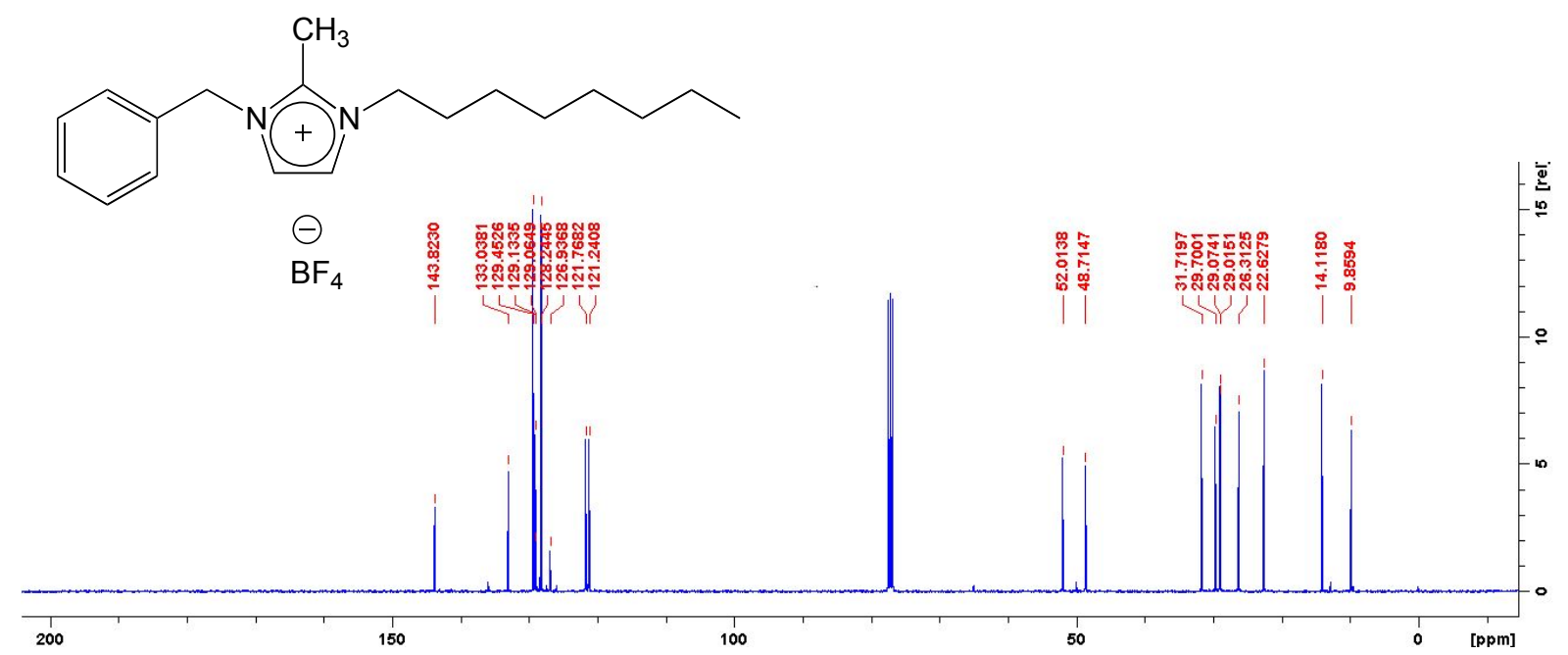


Figure S44. ${ }^{19} \mathrm{~F}-\mathrm{NMR}$ spectrum for IL product [BMO-im] $\mathrm{BF}_{4}$

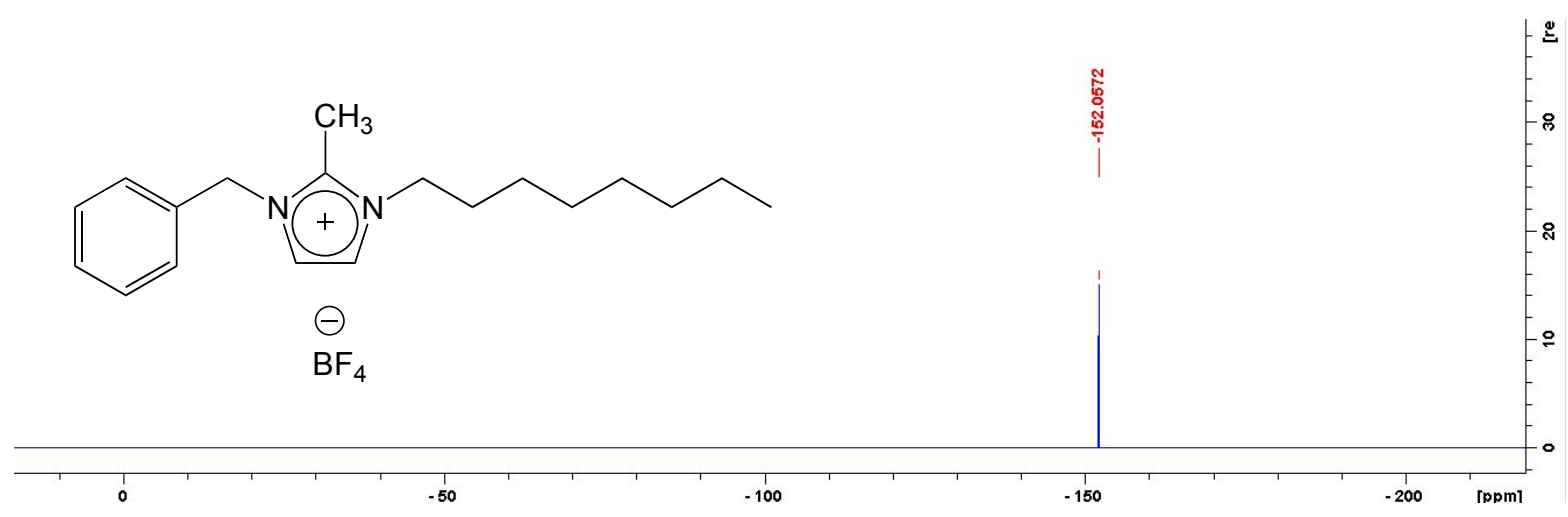

Figure S45. ${ }^{11} \mathrm{~B}-\mathrm{NMR}$ spectrum for IL product [BMO-im] $\mathrm{BF}_{4}$

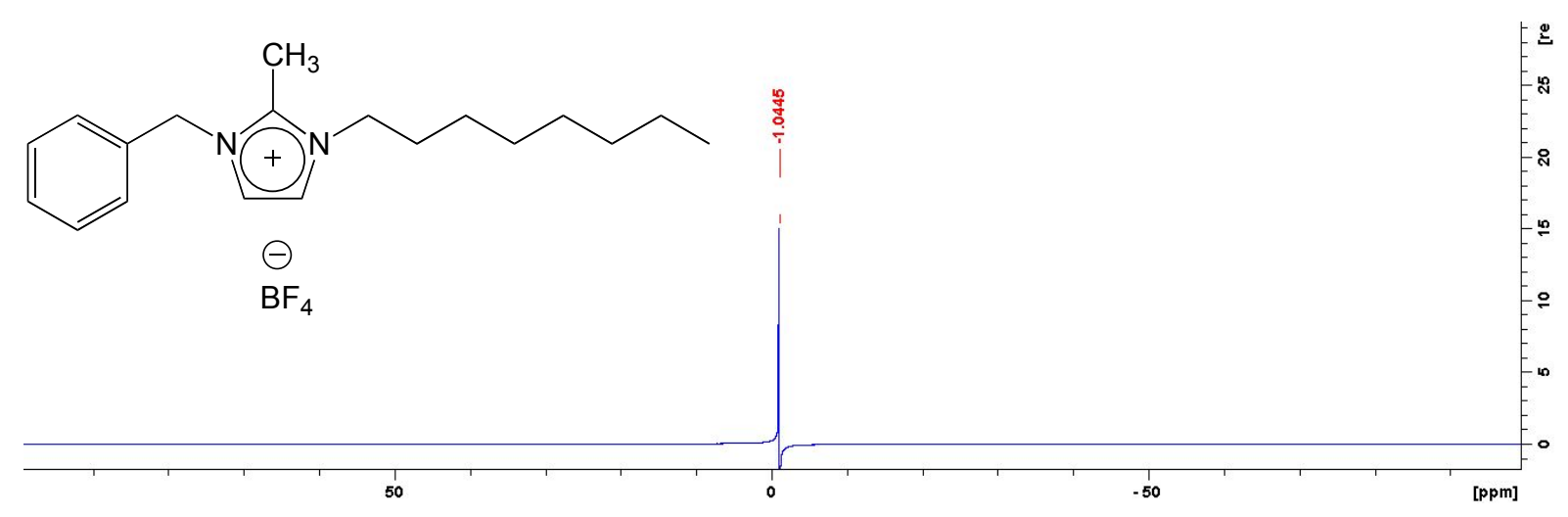

Figure S46. ${ }^{1} \mathrm{H}-\mathrm{NMR}$ spectrum for IL product [BMO-im] $\mathrm{PF}_{6}$

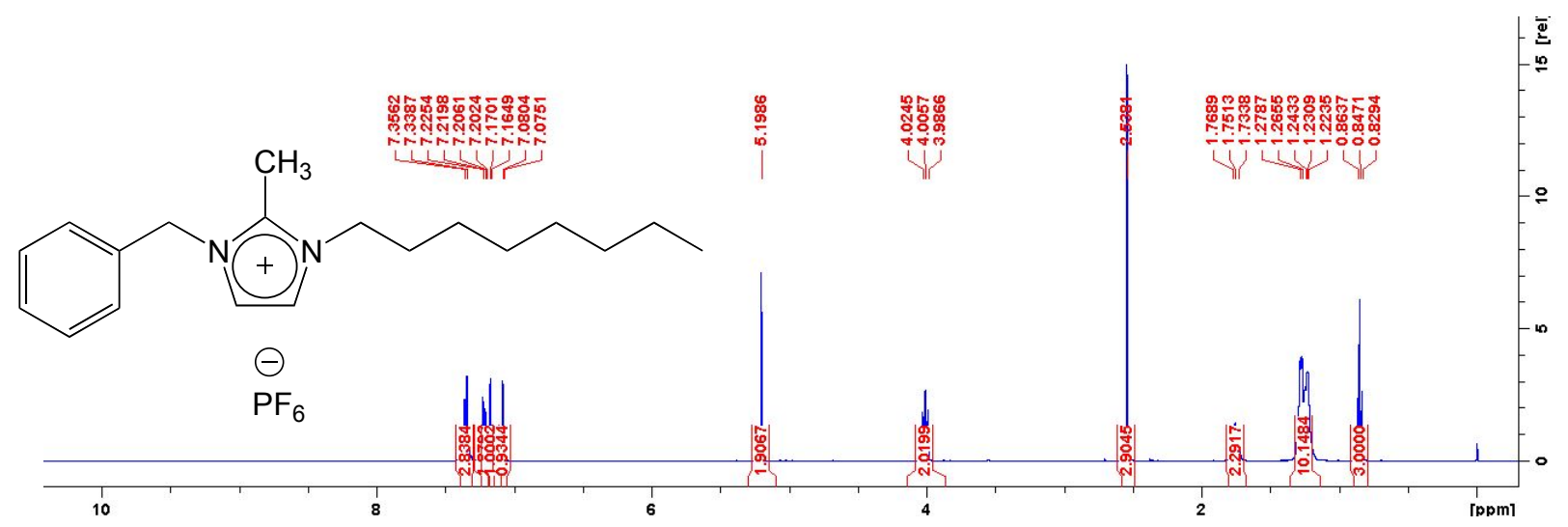




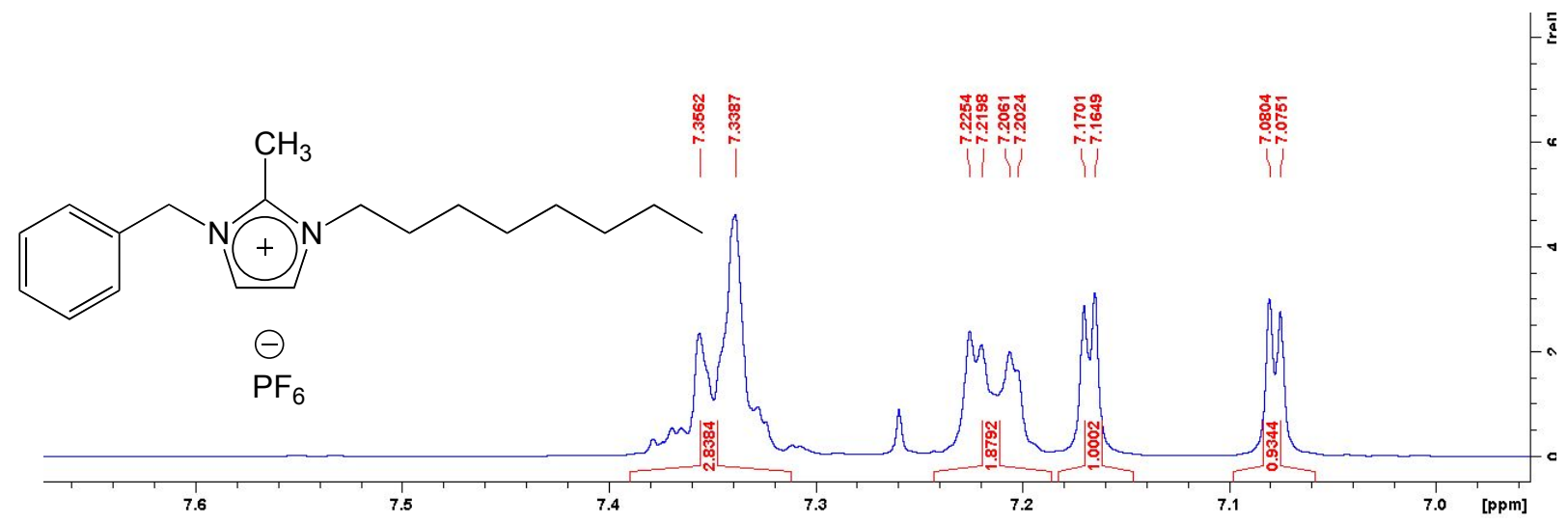

Figure S47. ${ }^{19} \mathrm{~F}-\mathrm{NMR}$ spectrum for IL product [BMO-im] $\mathrm{PF}_{6}$

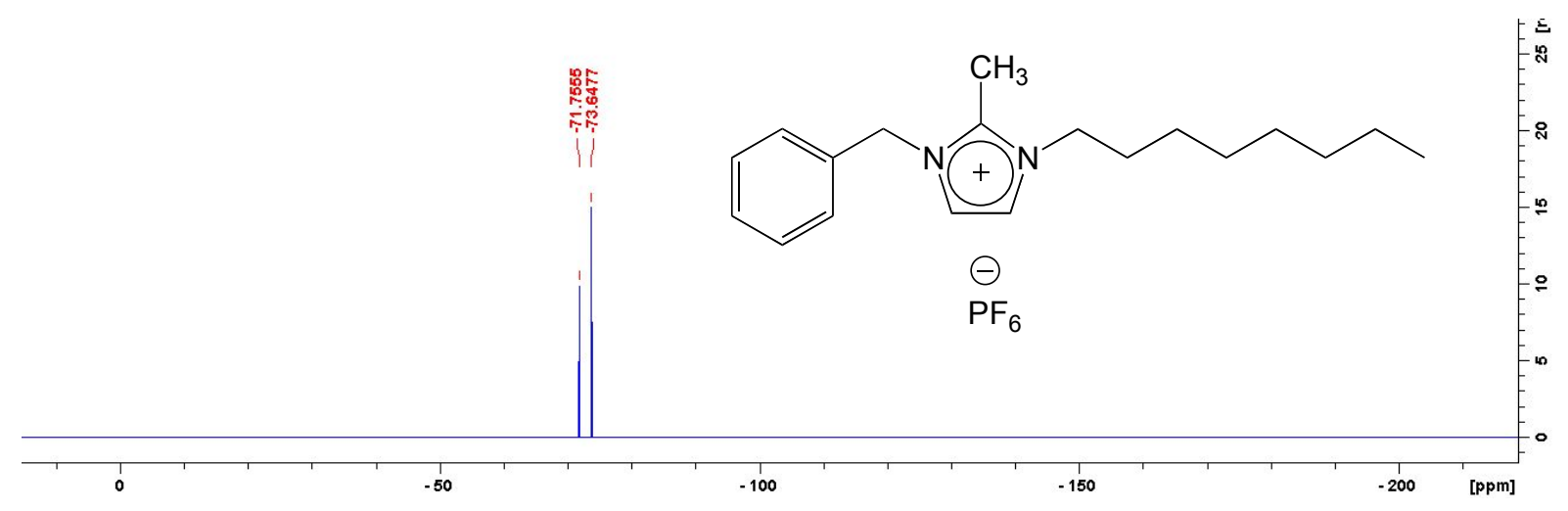

Figure S48. ${ }^{31} \mathrm{P}-\mathrm{NMR}$ spectrum for IL product [BMO-im] $\mathrm{PF}_{6}$

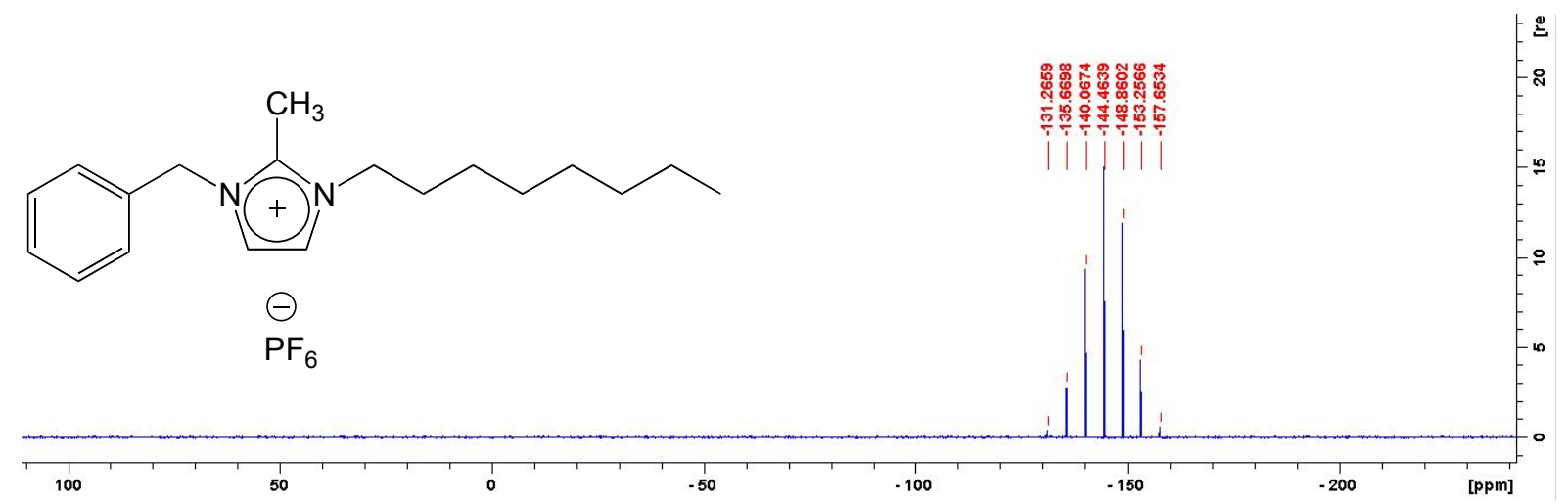


Figure S49. ${ }^{1} \mathrm{H}-\mathrm{NMR}$ spectrum for IL product [BMO-im] $\mathrm{NTf}_{2}$
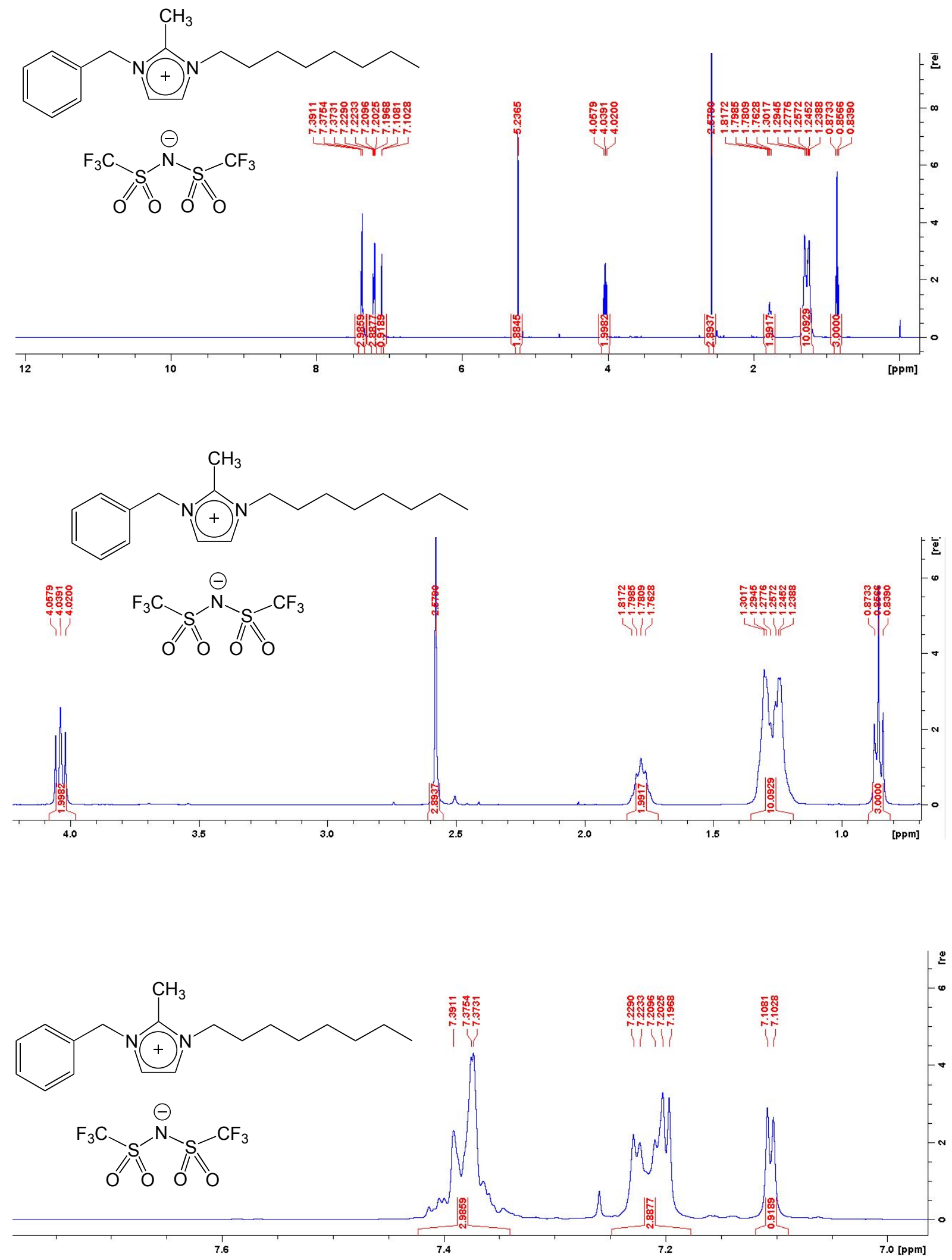

Figure S50. ${ }^{13} \mathrm{C}-\mathrm{NMR}$ spectrum for IL product [BMO-im] NTf ${ }_{2}$ 


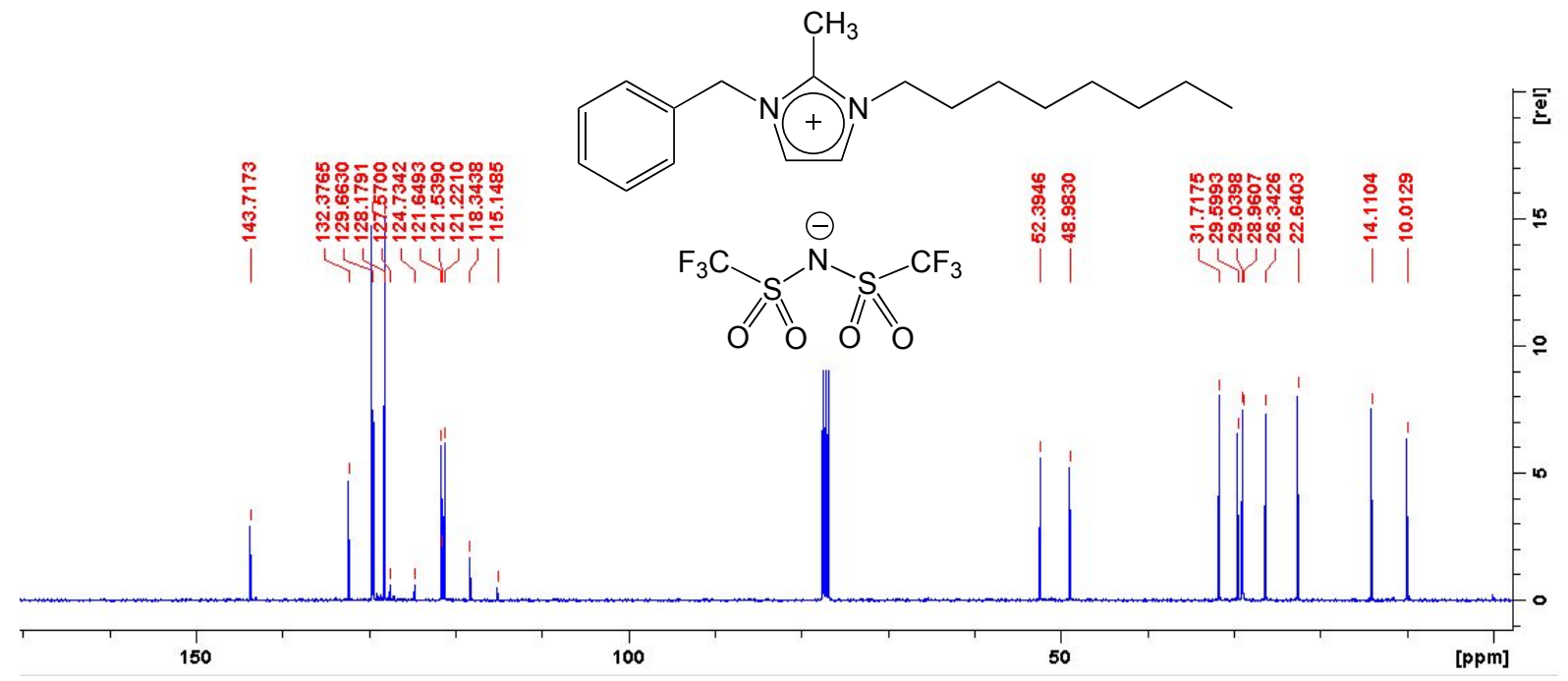

Figure S51. ${ }^{19} \mathrm{~F}-\mathrm{NMR}$ spectrum for IL product [BMO-im] $\mathrm{NTf}_{2}$

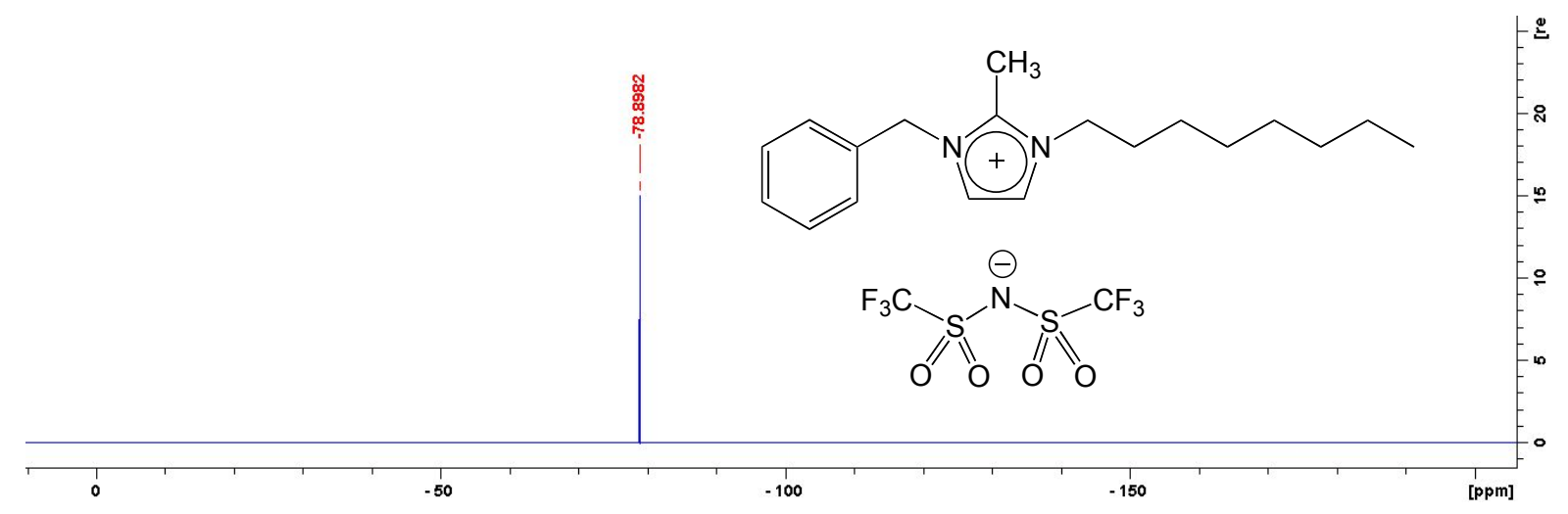


Figure S52. COSY spectrum for IL product [DBU-M-TMSi] NTf ${ }_{2}$

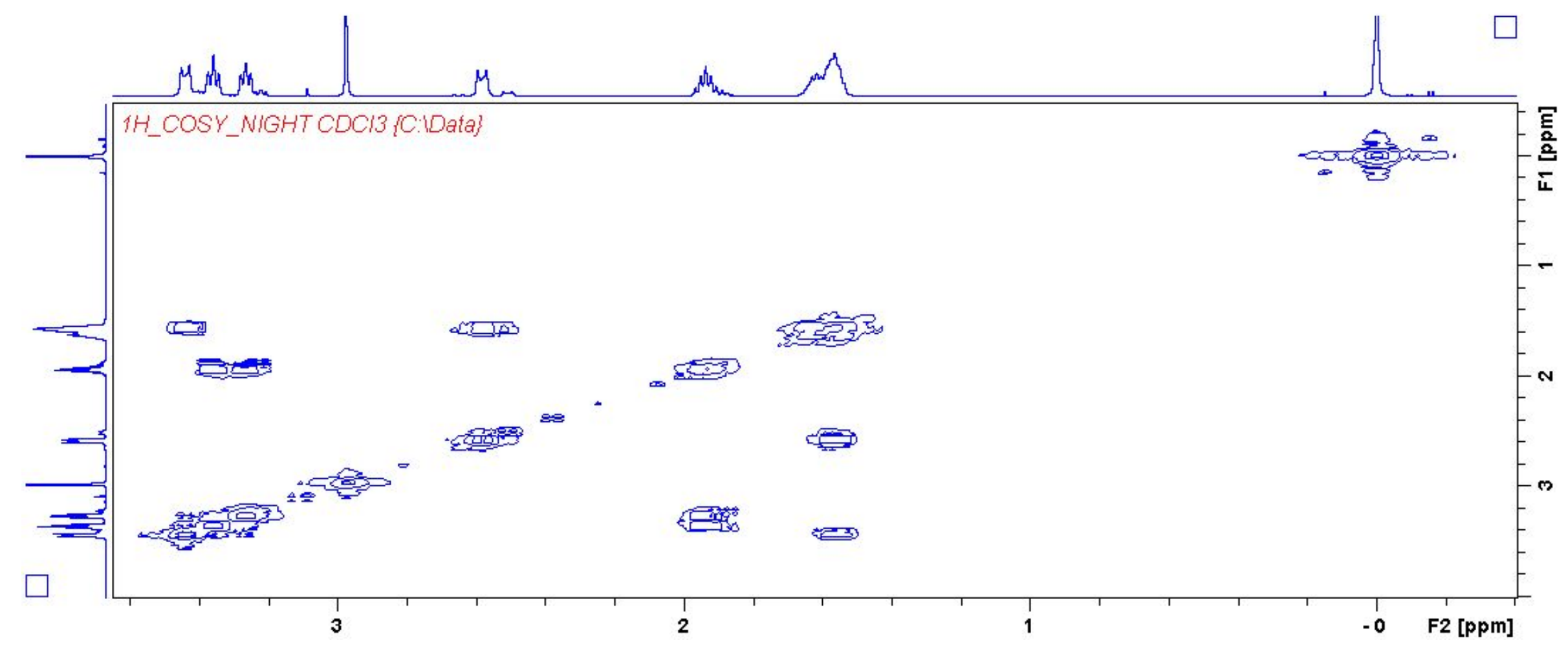


Figure S53. HMBC spectrum for IL product [DBU-M-TMSi] NTf

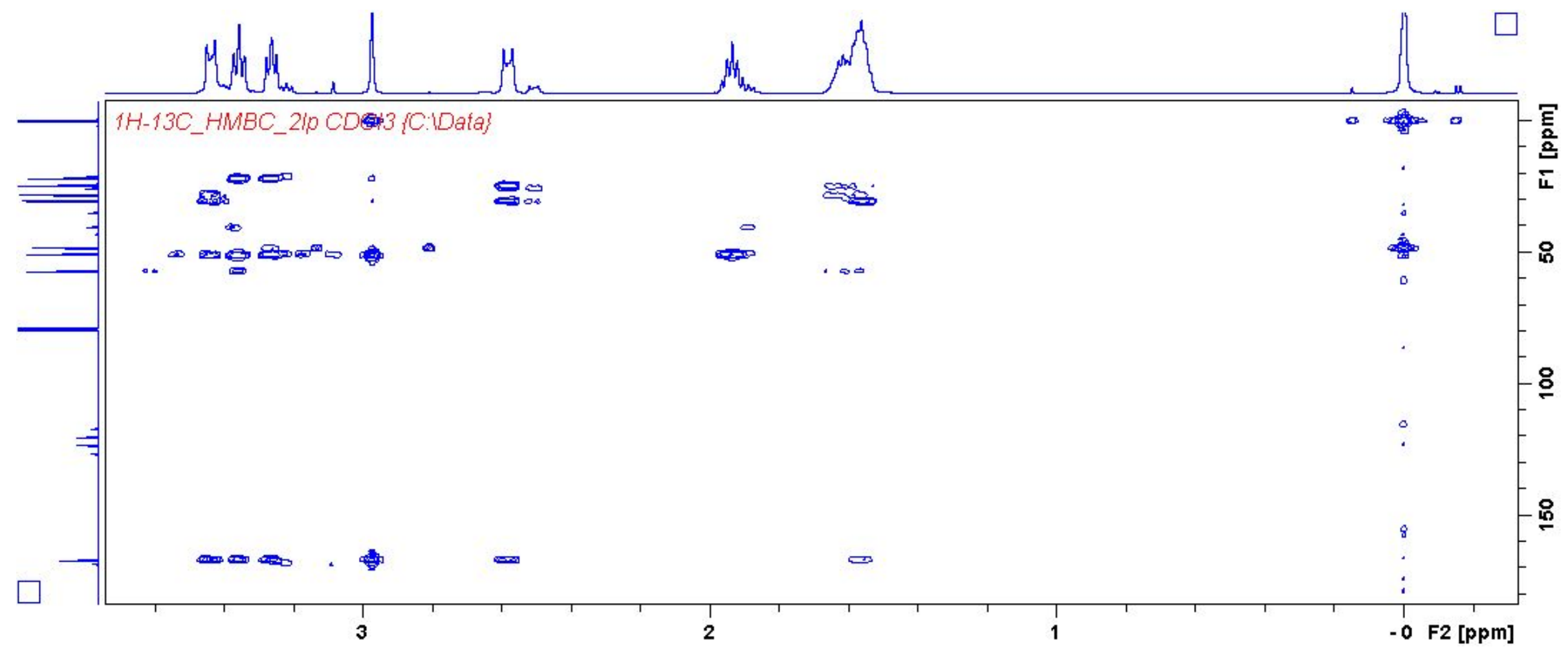


Figure S54. HSQC spectrum for IL product [DBU-M-TMSi] NTf 2

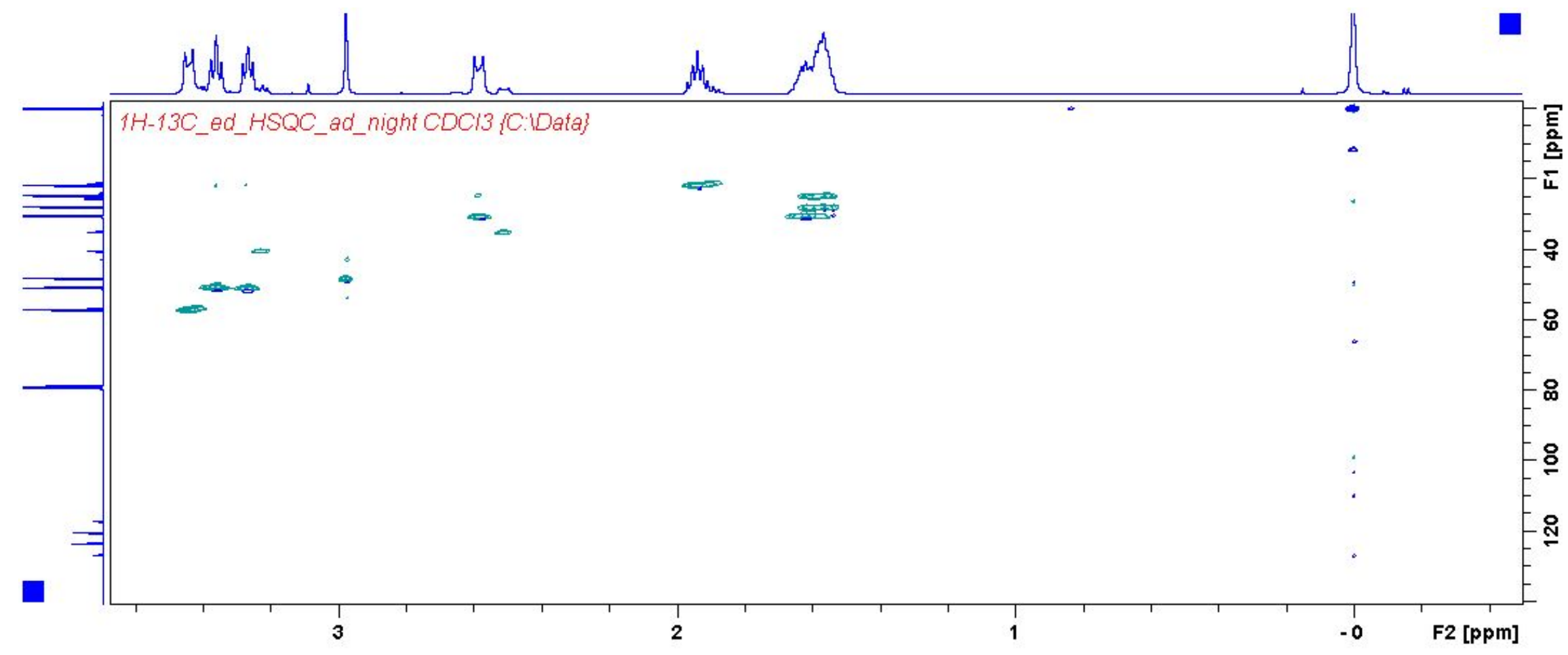


Figure S55. COSY spectrum for IL product [BM-M-TMSi-im] NTf $f_{2}$

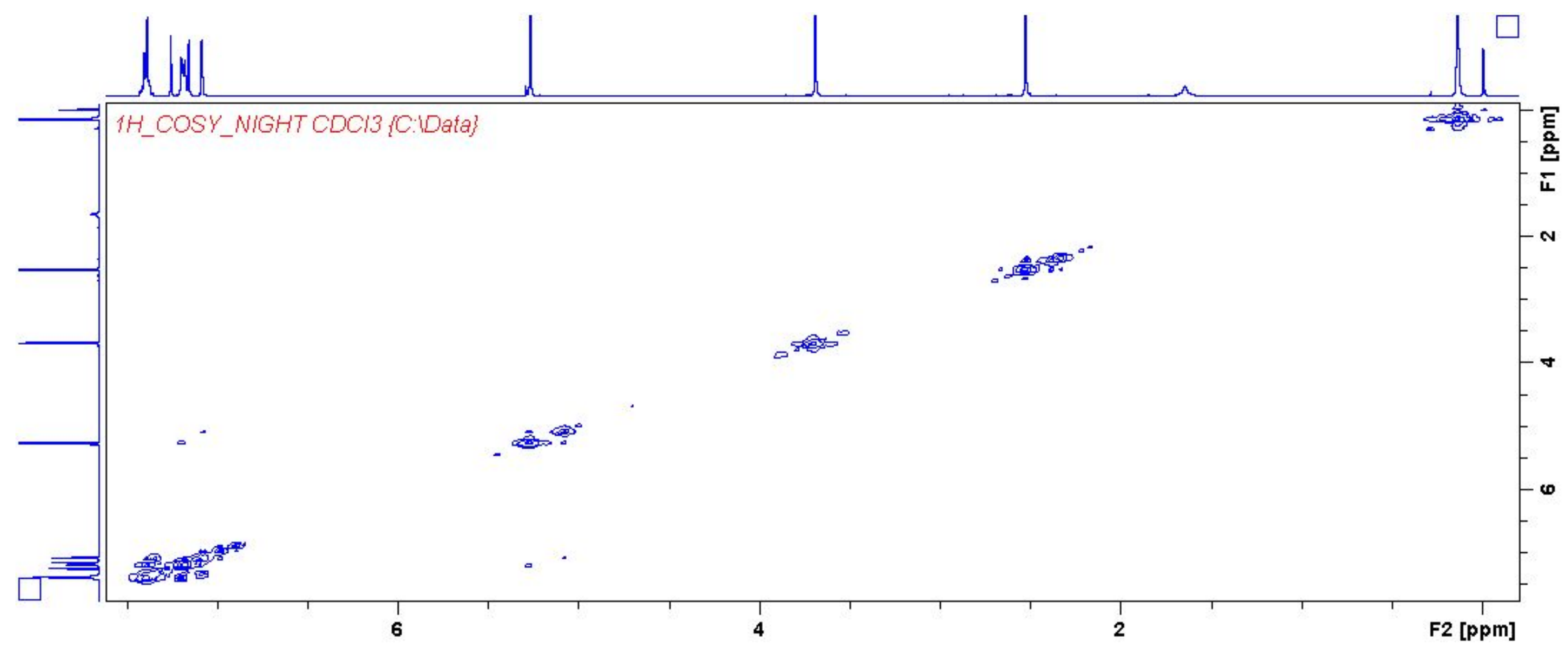


Figure S56. HMBC spectrum for IL product [BM-M-TMSi-im] NTf

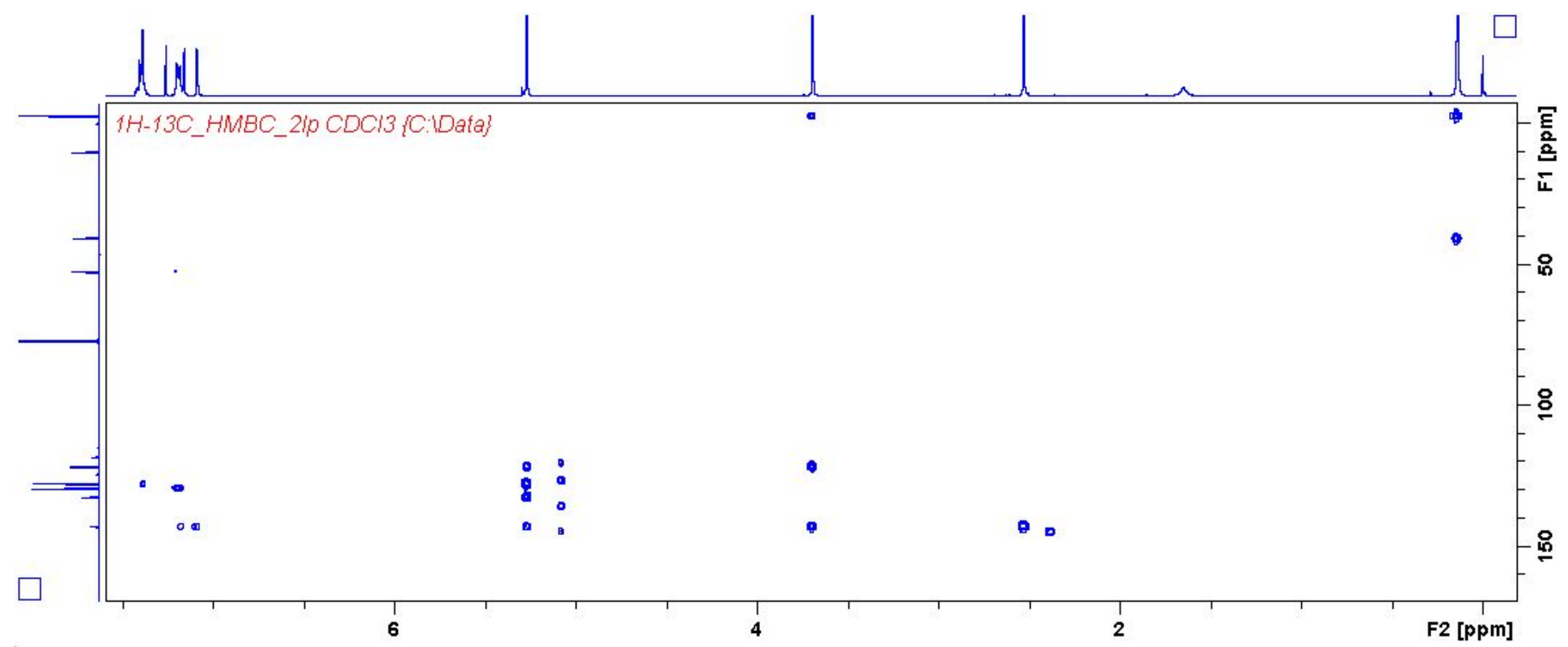


Figure S57. HSQC spectrum for IL product [BM-M-TMSi-im] NTf $f_{2}$

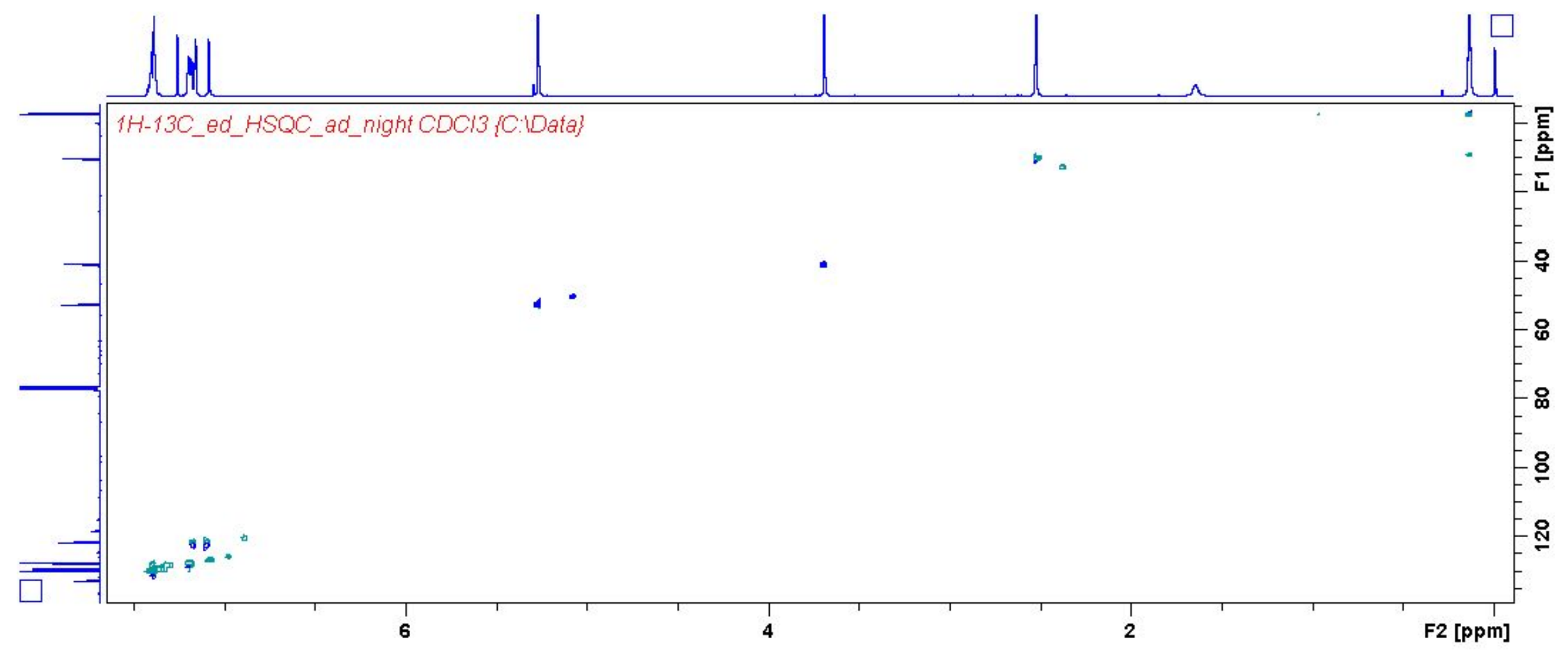


Figure S58. COSY spectrum for IL product [DMMB-im] NTf ${ }_{2}$

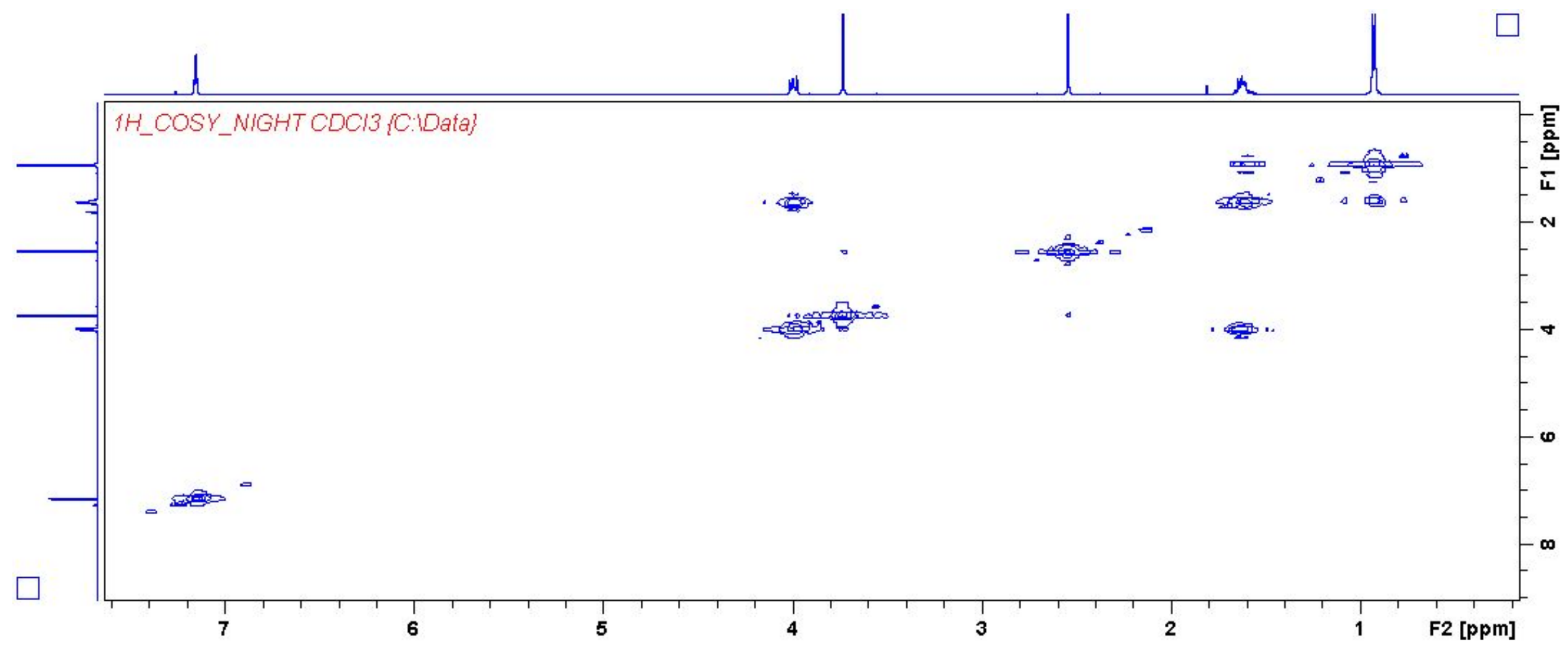


Figure S59. HMBC spectrum for IL product [DMMB-im] NTf $f_{2}$

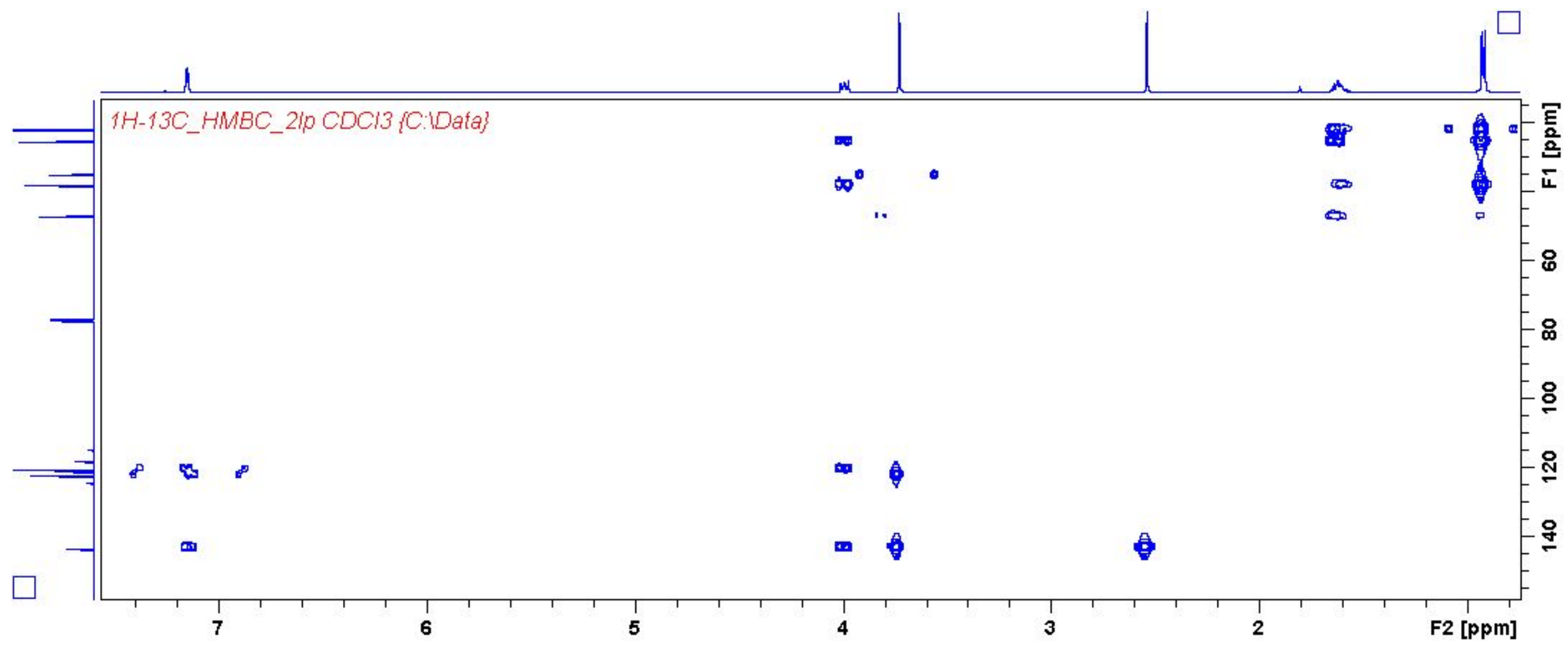


Figure S60. HSQC spectrum for IL product [DMMB-im] $\mathrm{NTf}_{2}$

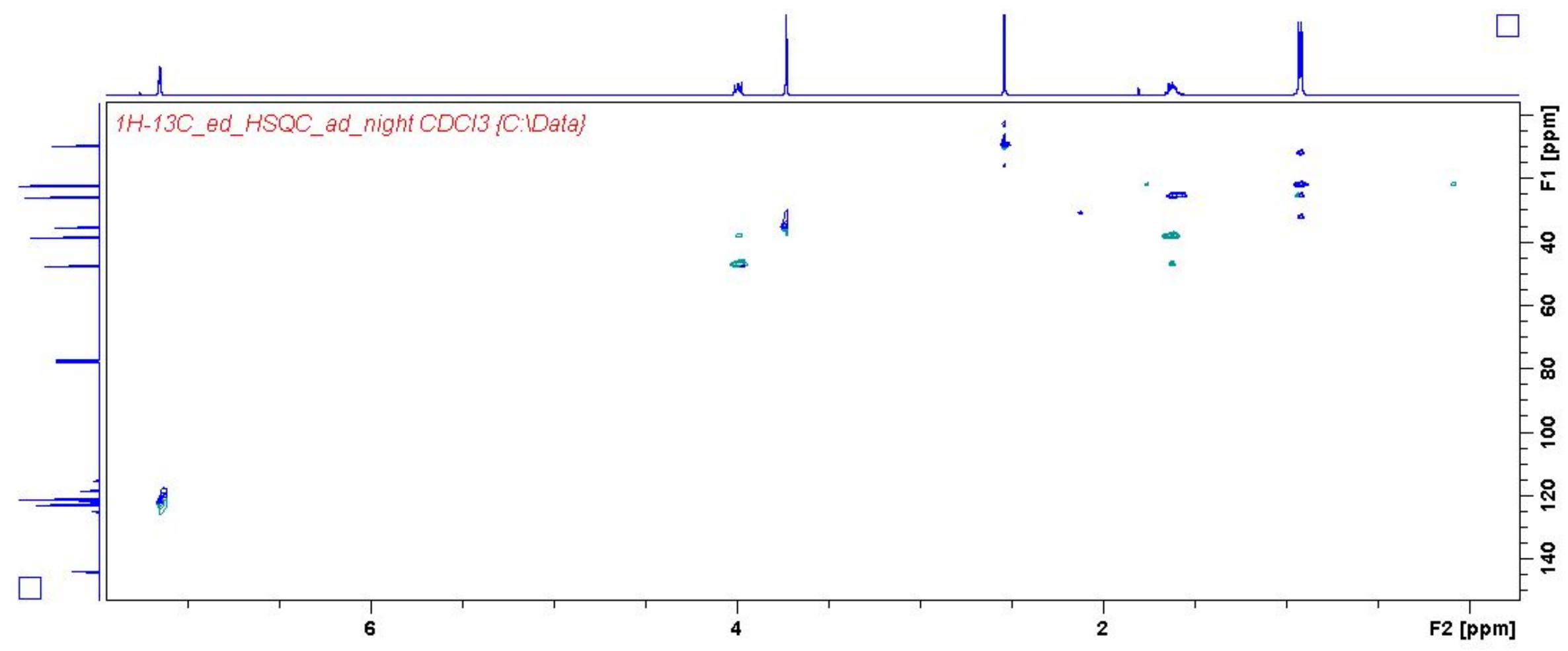


Figure S61. COSY spectrum for IL product [DMMB-im] $\mathrm{BF}_{4}$

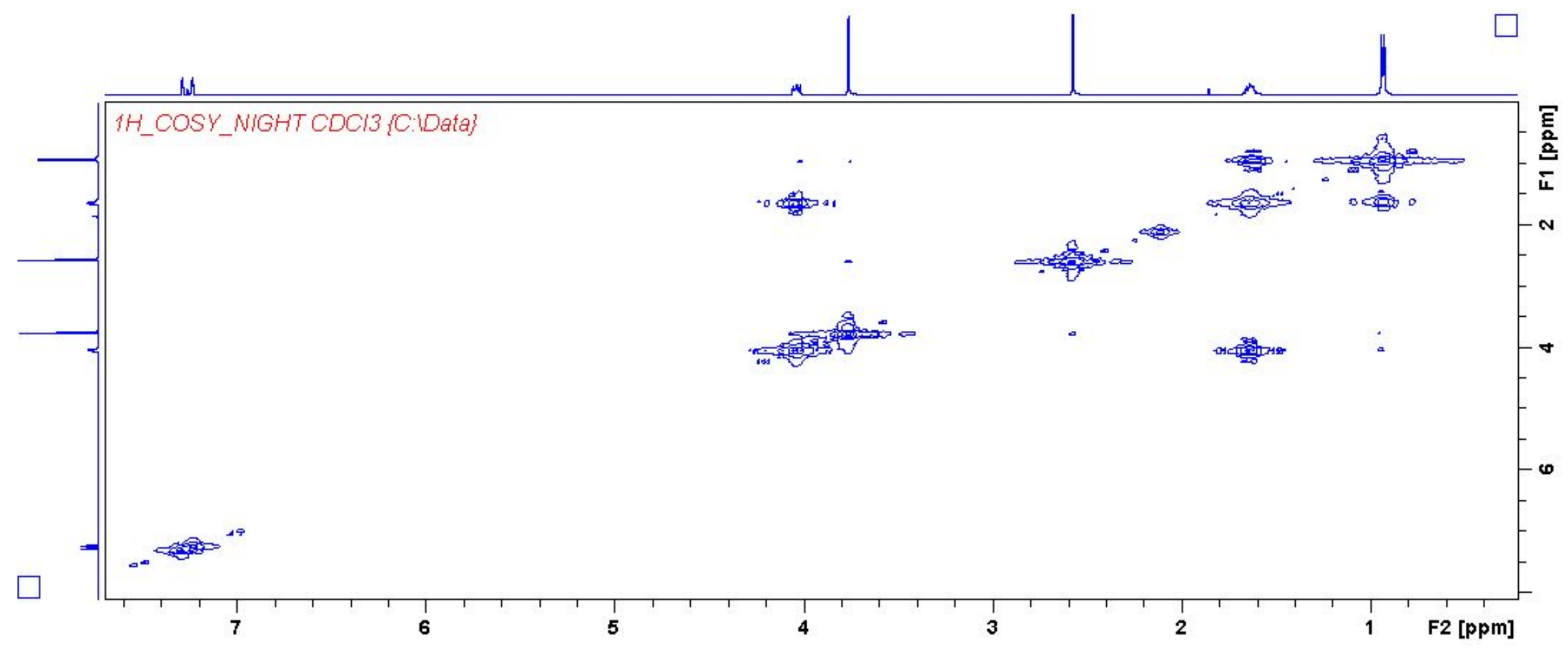


Figure S62. HMBC spectrum for IL product [DMMB-im] $\mathrm{BF}_{4}$

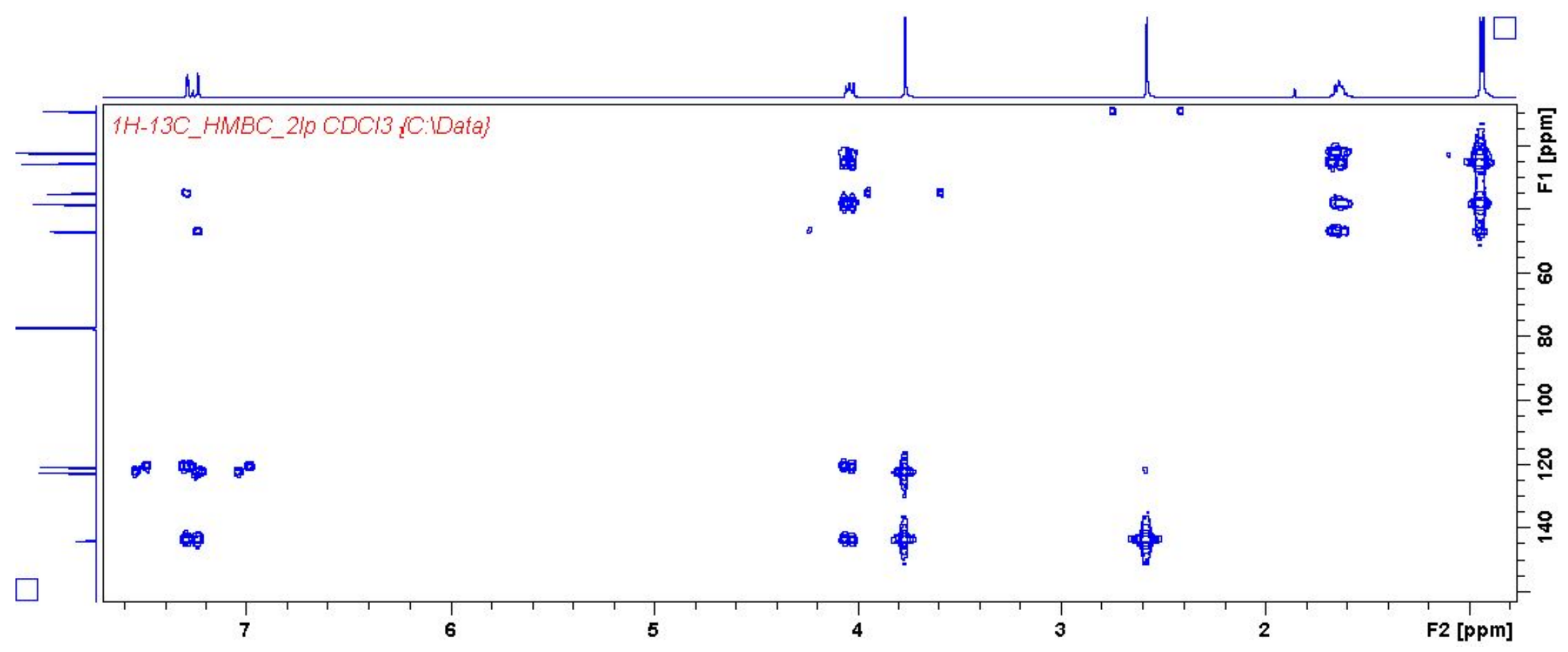


Figure S63. HSQC spectrum for IL product [DMMB-im] $\mathrm{BF}_{4}$

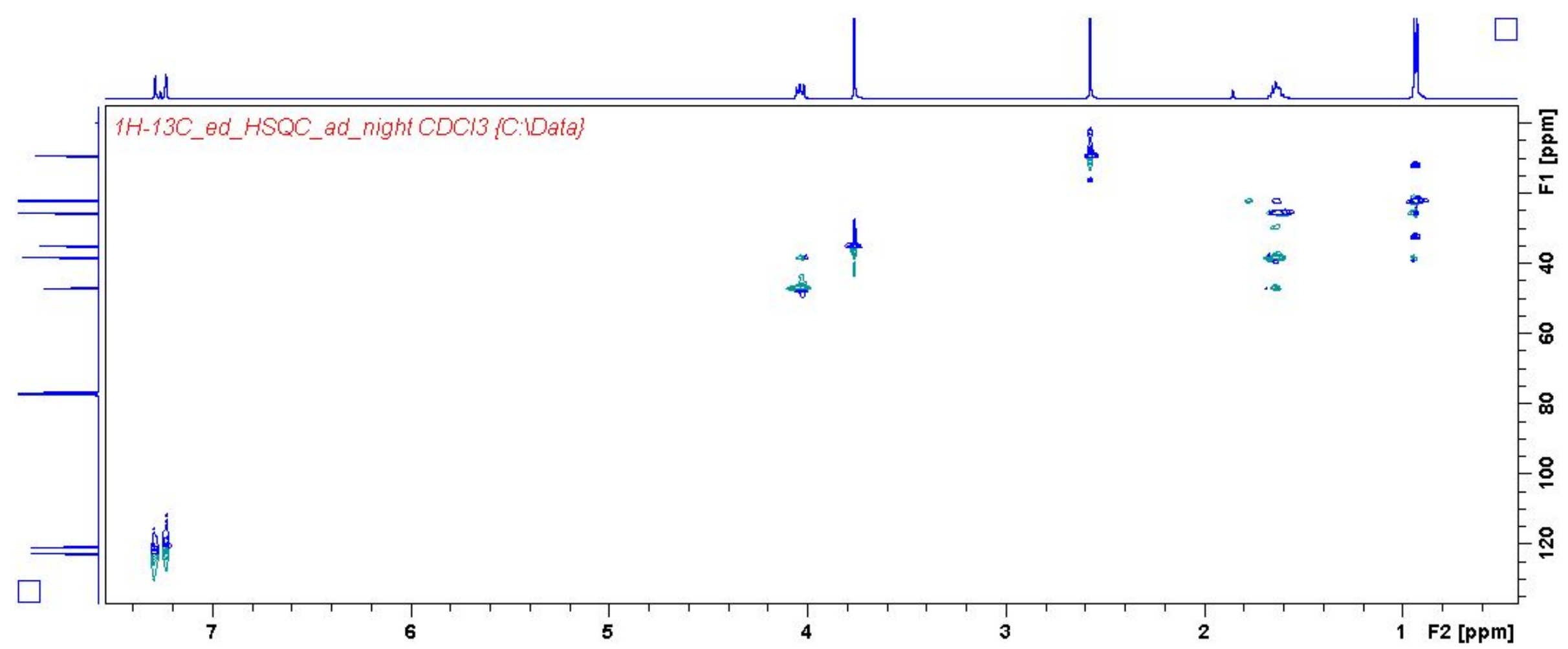


Figure 664 . COSY spectrum for IL product $\left[\mathrm{BP}_{888}\right] \mathrm{NTf}_{2}$

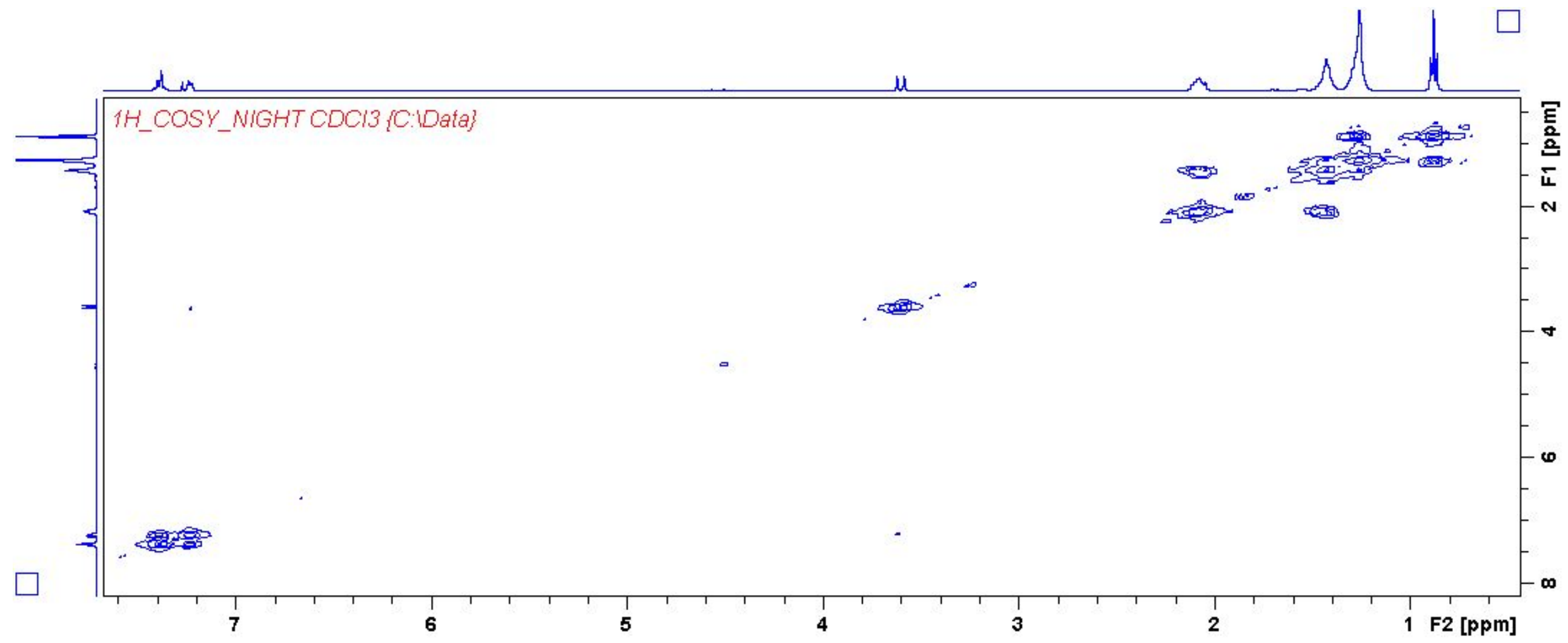


Figure $\mathrm{S65}$. HMBC spectrum for IL product $\left[\mathrm{BP}_{888}\right] \mathrm{NTf}_{2}$

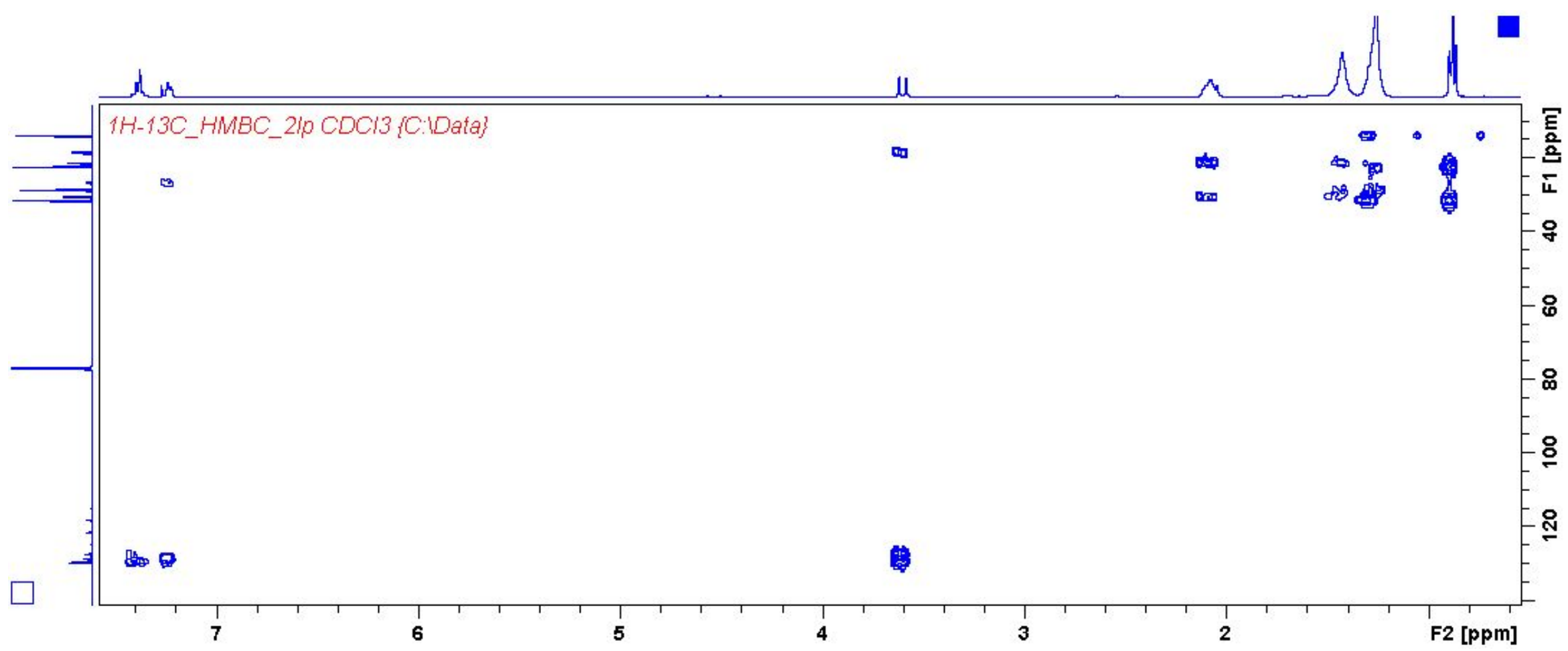


Figure S66. HSQC spectrum for IL product $\left[\mathrm{BP}_{888}\right] \mathrm{NTf}_{2}$

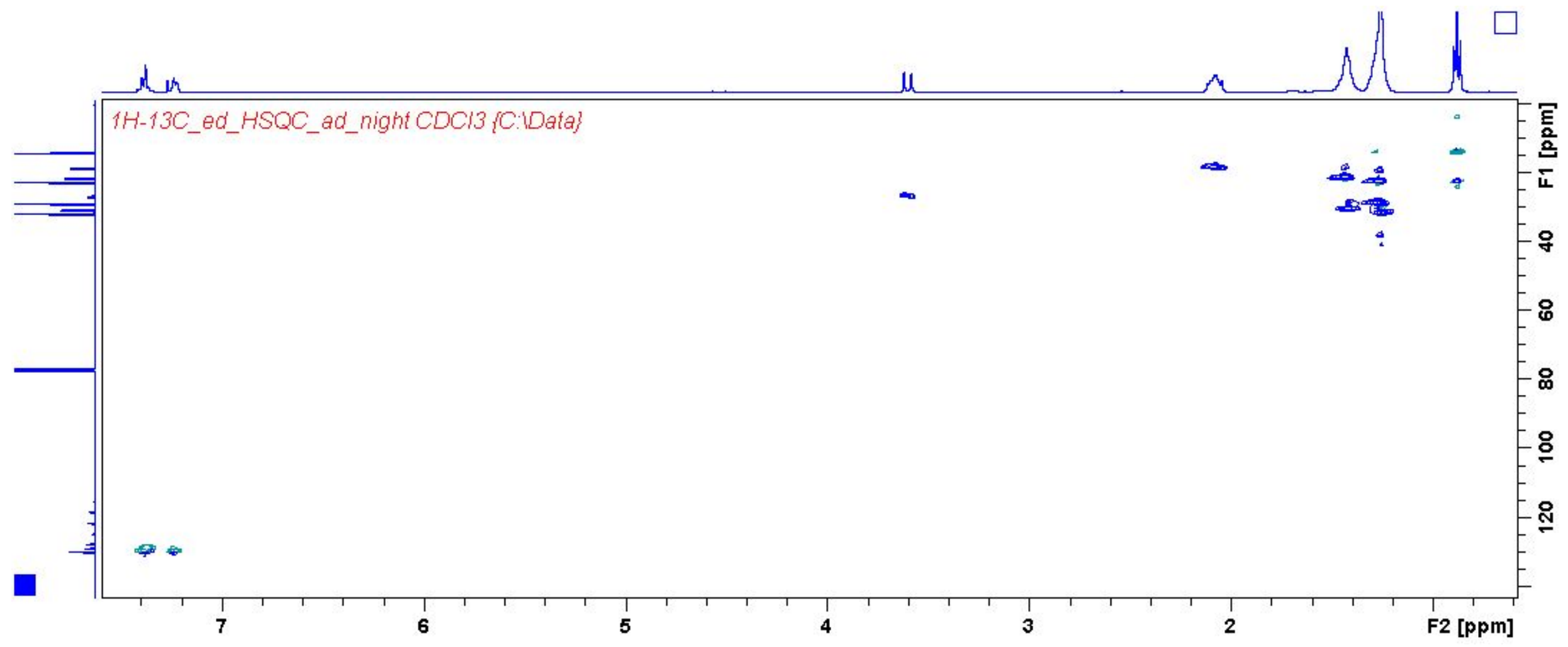


Figure S67. COSY spectrum for IL product [ABM-im] $\mathrm{NTf}_{2}$

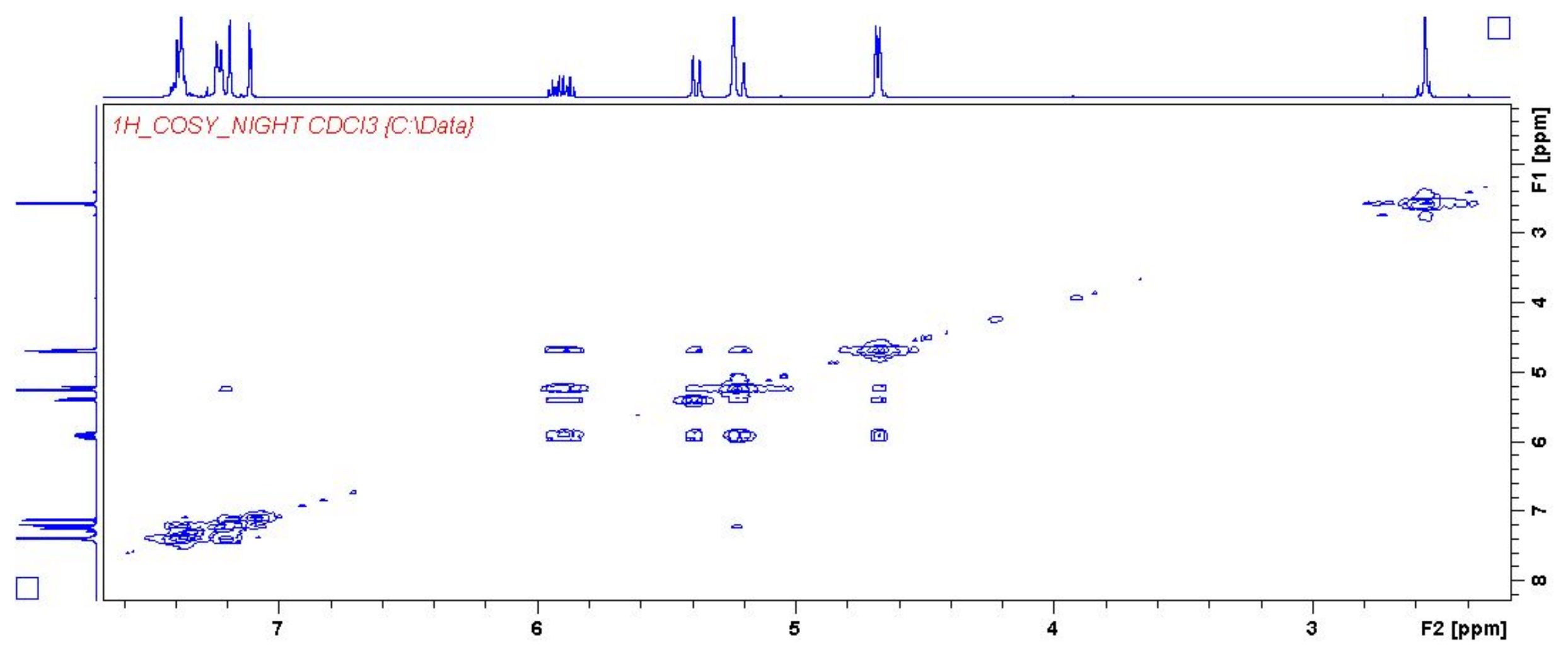


Figure S68. HMBC spectrum for IL product [ABM-im] $\mathrm{NTf}_{2}$

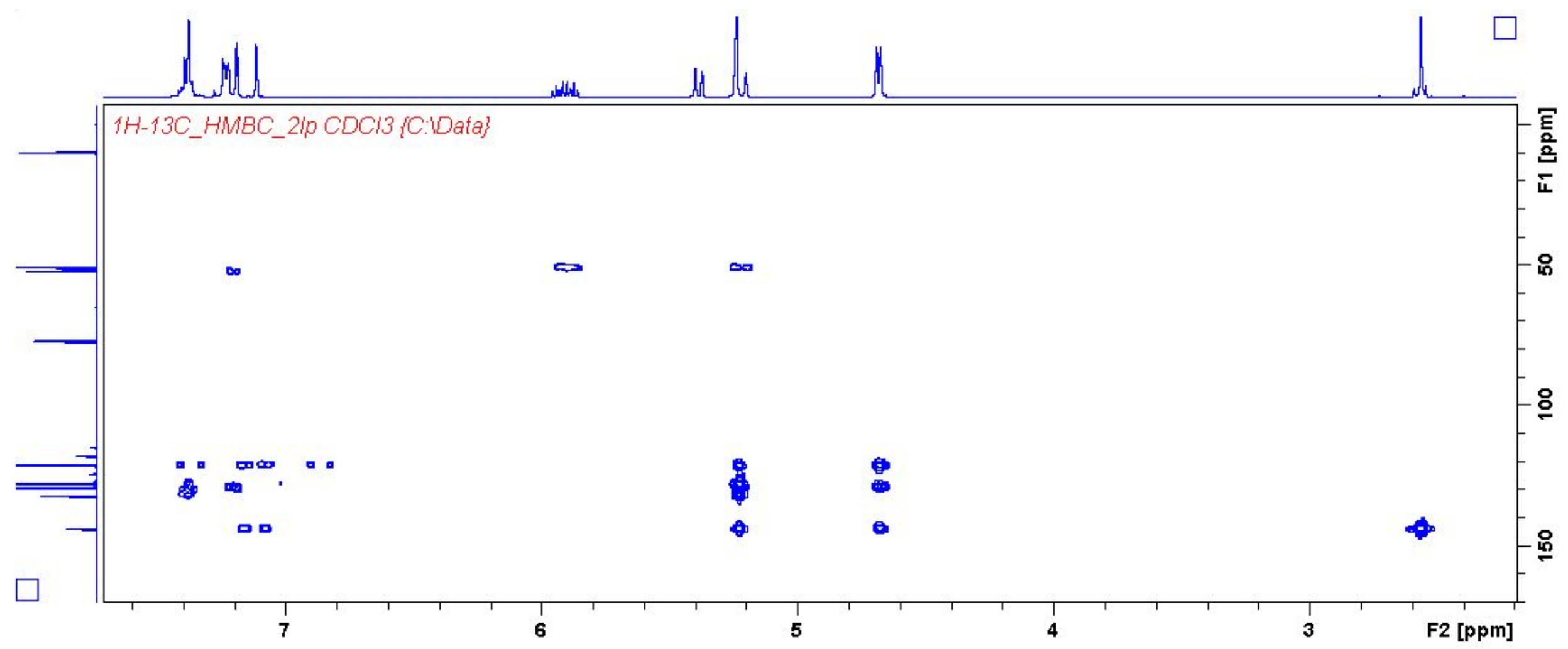


Figure S69. HSQC spectrum for IL product [ABM-im] $\mathrm{NTf}_{2}$

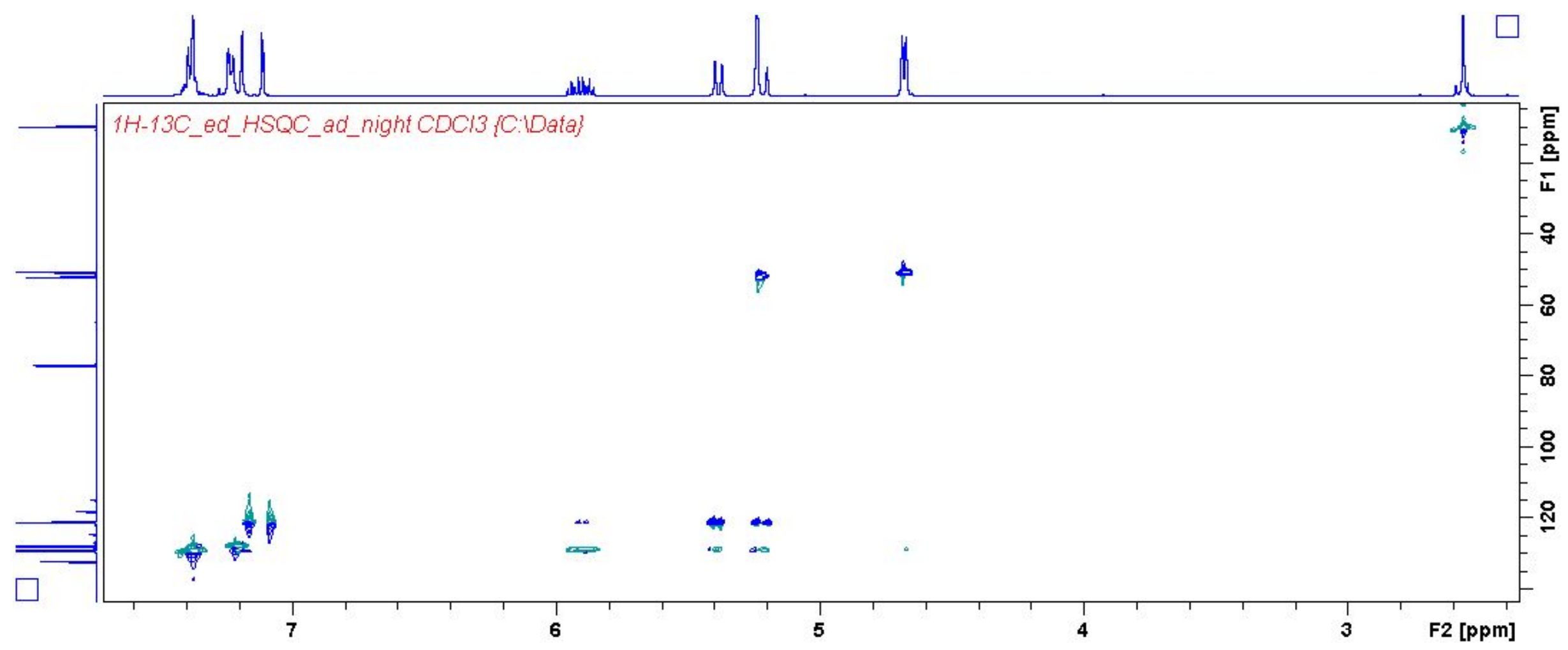


Figure S70. COSY spectrum for IL product [BMO-im] $\mathrm{NTf}_{2}$

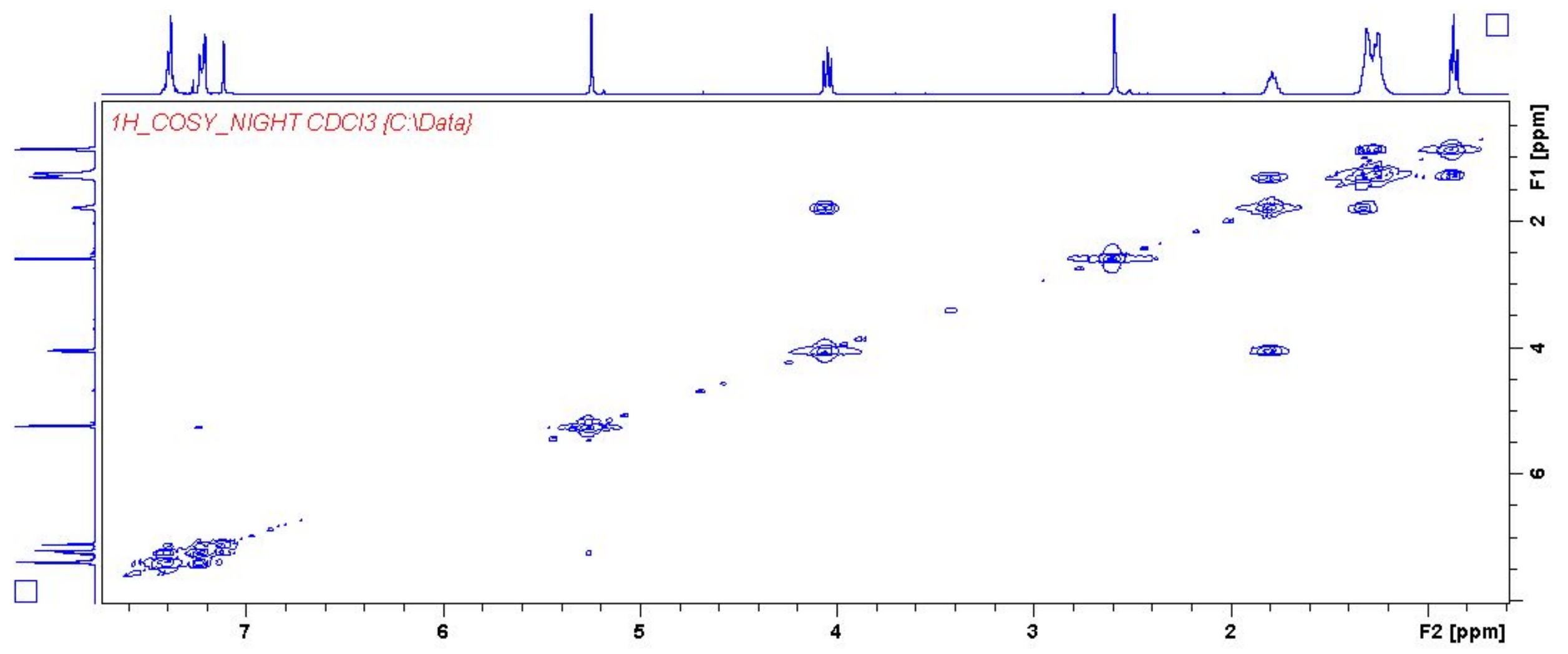


Figure S71. HMBC spectrum for IL product [BMO-im] $\mathrm{NTf}_{2}$

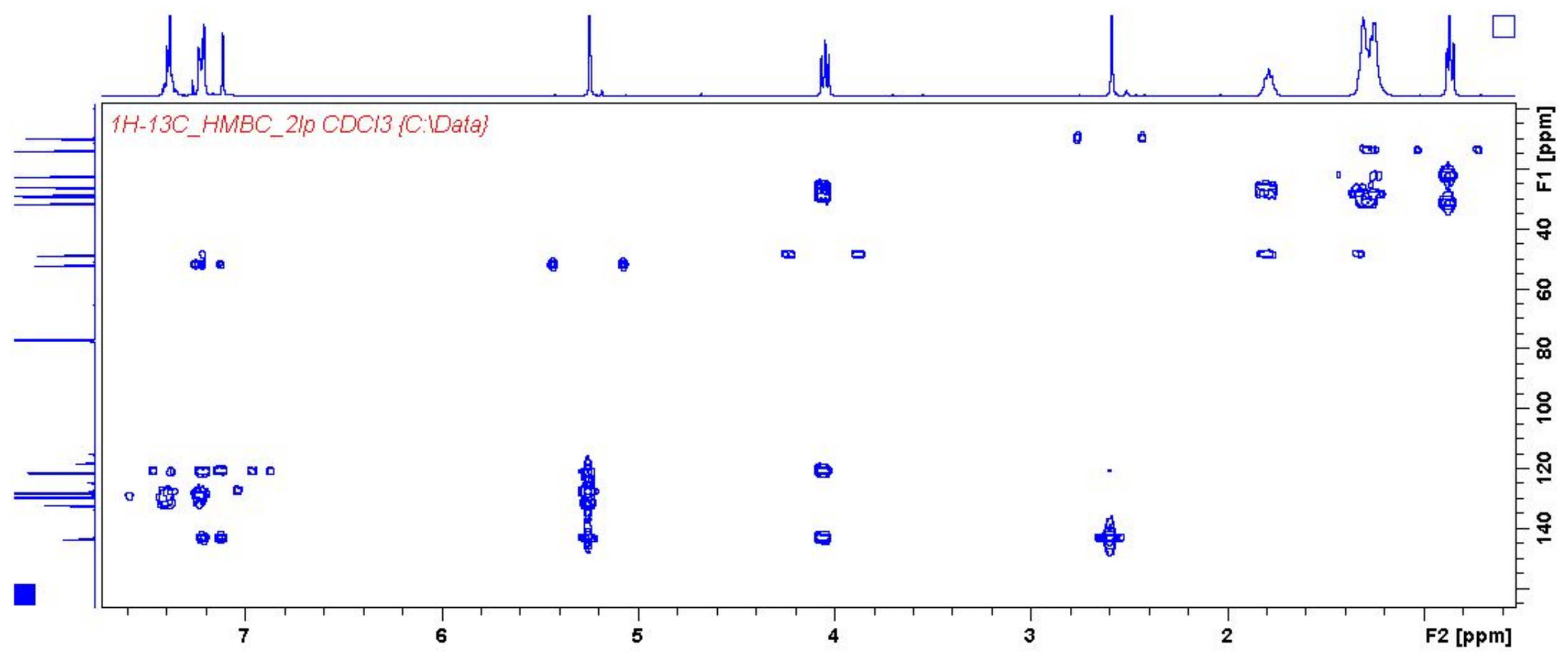


Figure S72. HSQC spectrum for IL product [BMO-im] $\mathrm{NTf}_{2}$

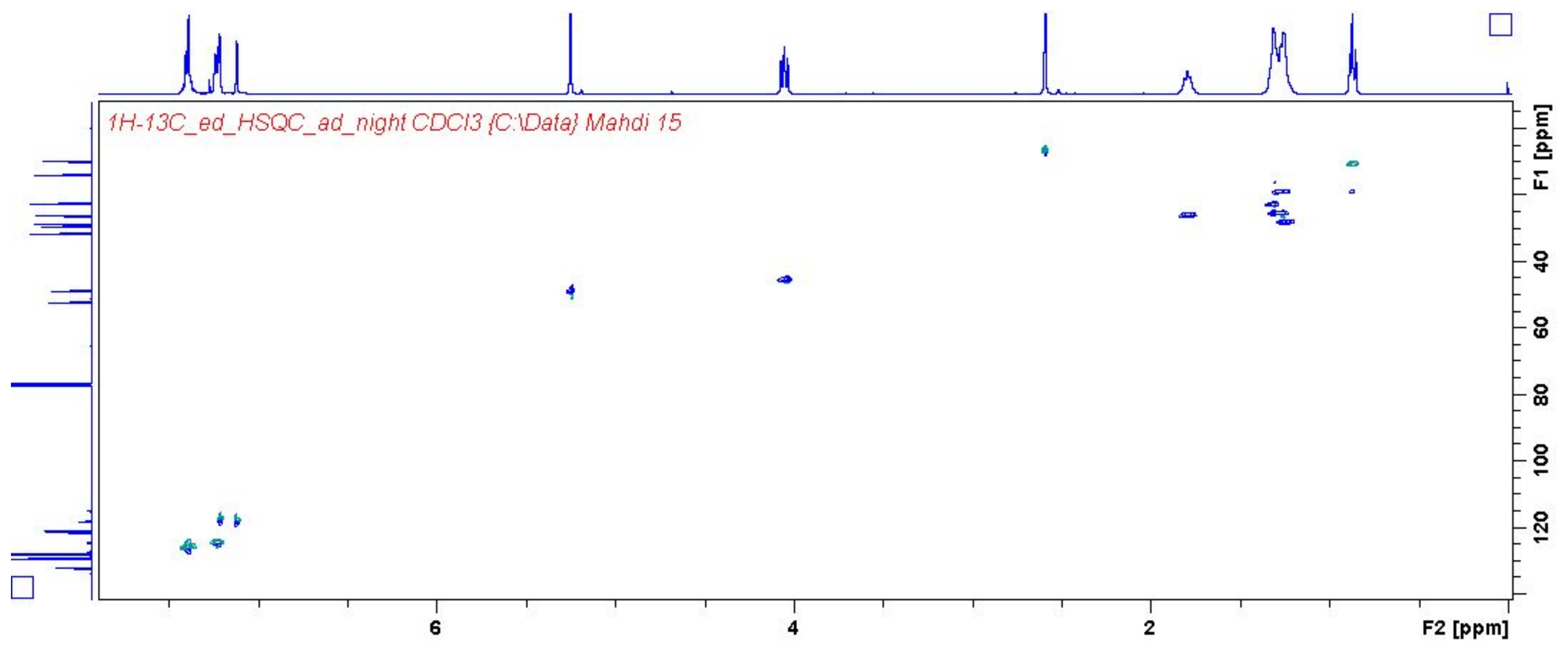


Figure S73. COSY spectrum for IL product [BMO-im] $\mathrm{PF}_{6}$

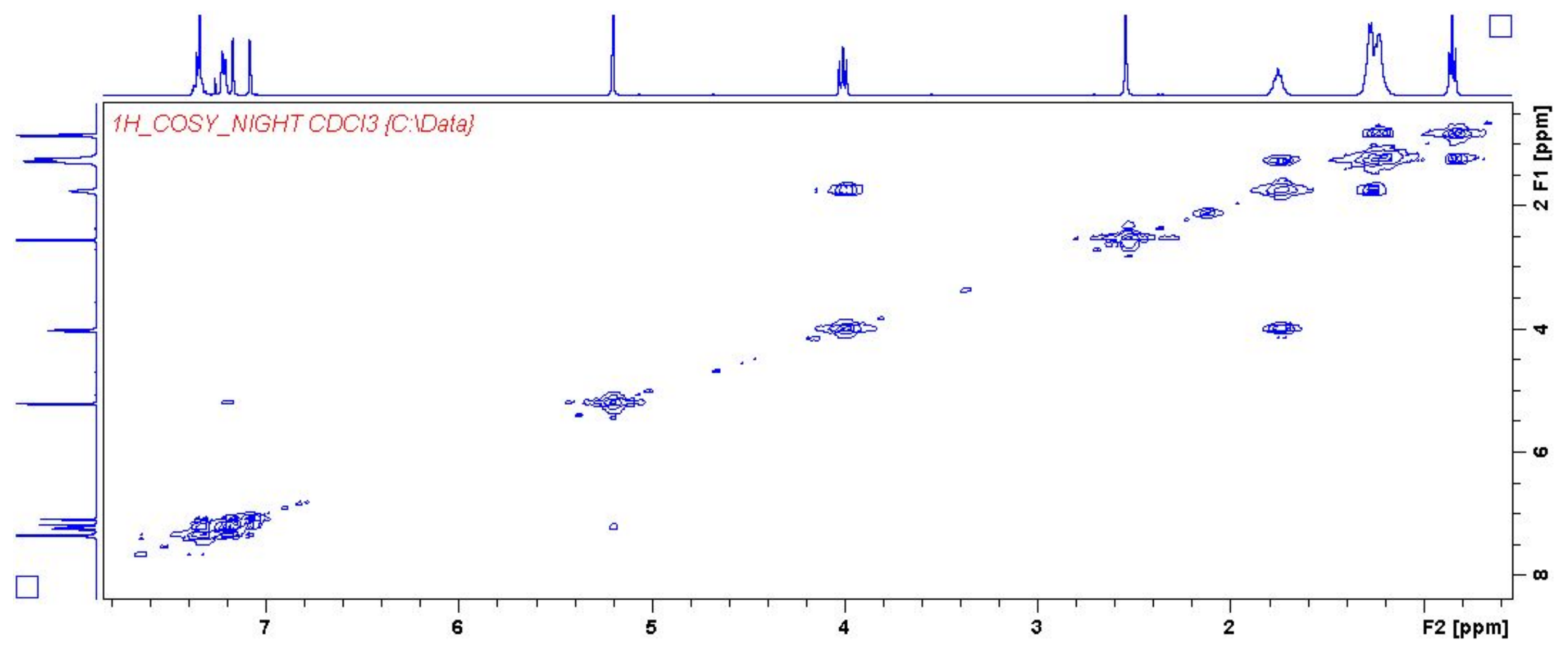


Figure S74. HMBC spectrum for IL product [BMO-im] $\mathrm{PF}_{6}$

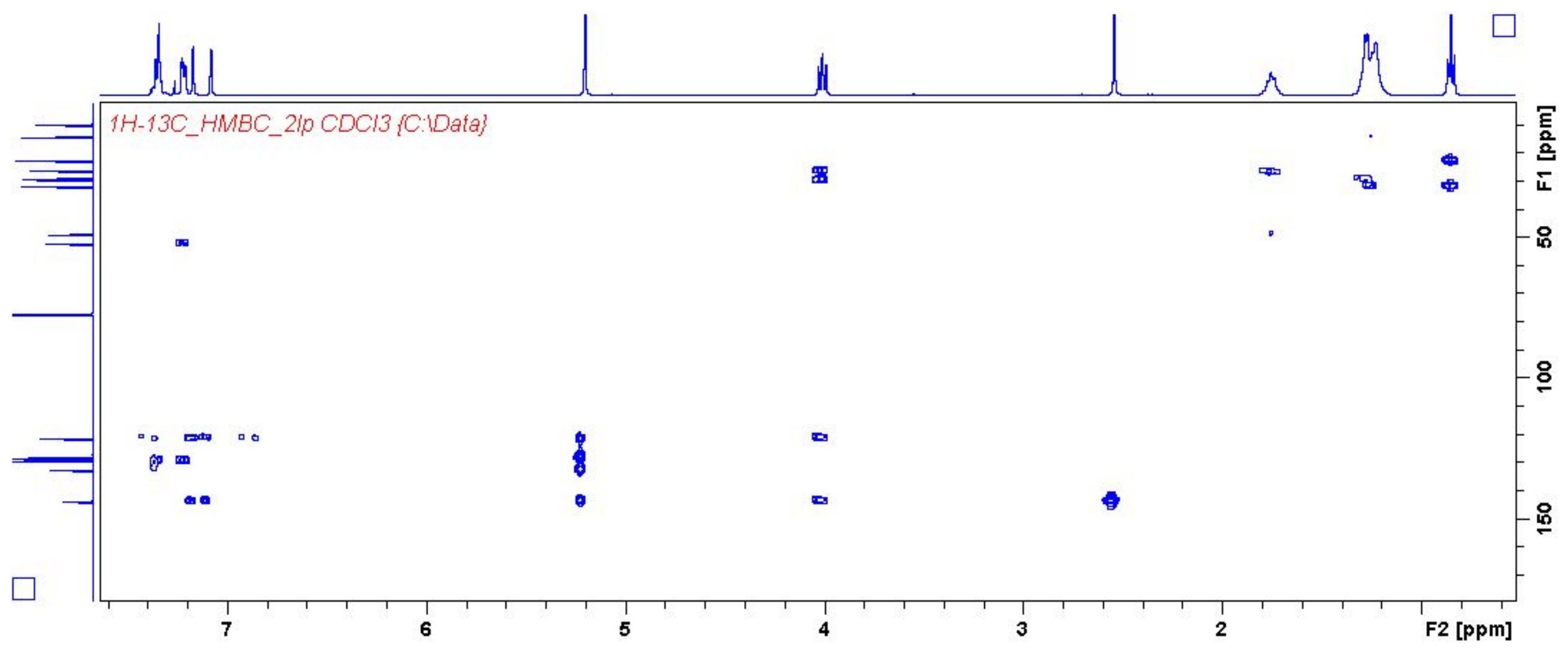


Figure S75. HSQCspectrum for IL product [BMO-im] $\mathrm{PF}_{6}$

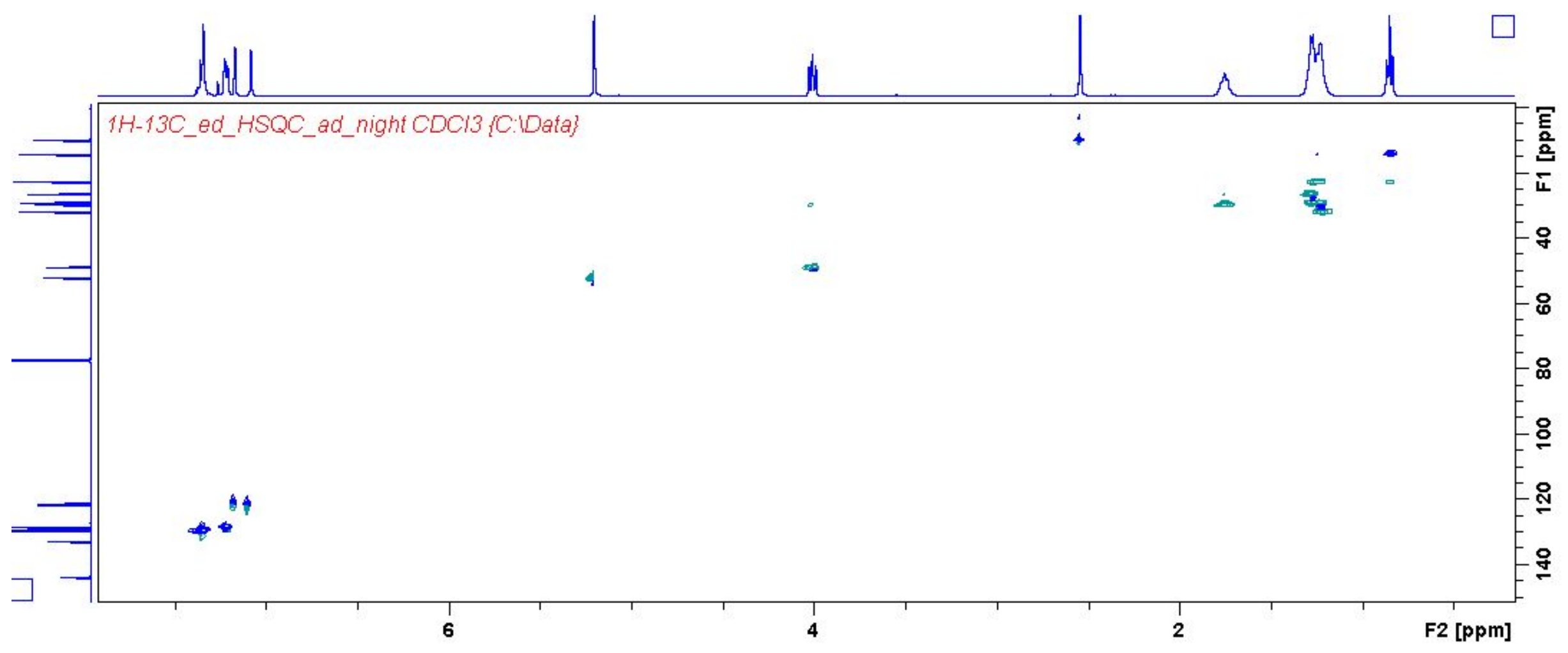


Figure S76. COSY spectrum for IL product [BMO-im] $\mathrm{BF}_{4}$

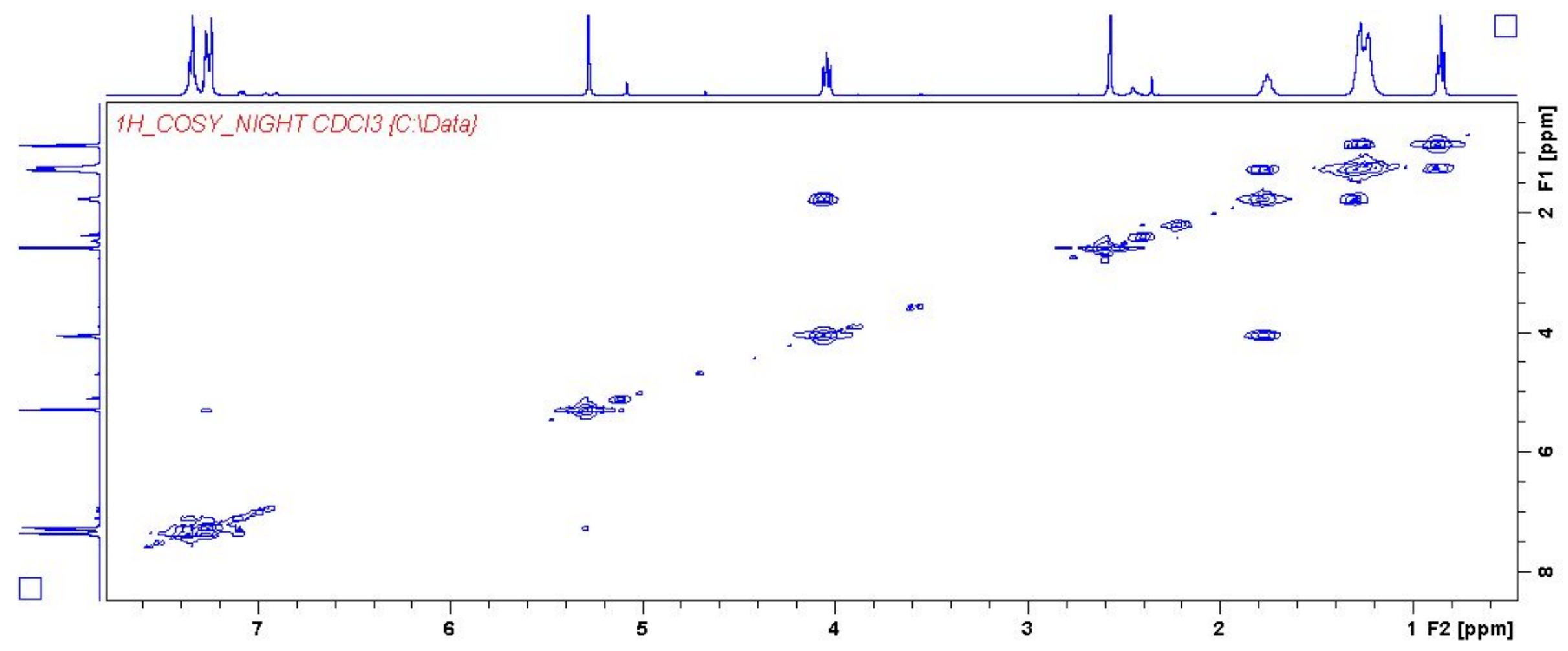


Figure S77. HMBC spectrum for IL product [BMO-im] $\mathrm{BF}_{4}$

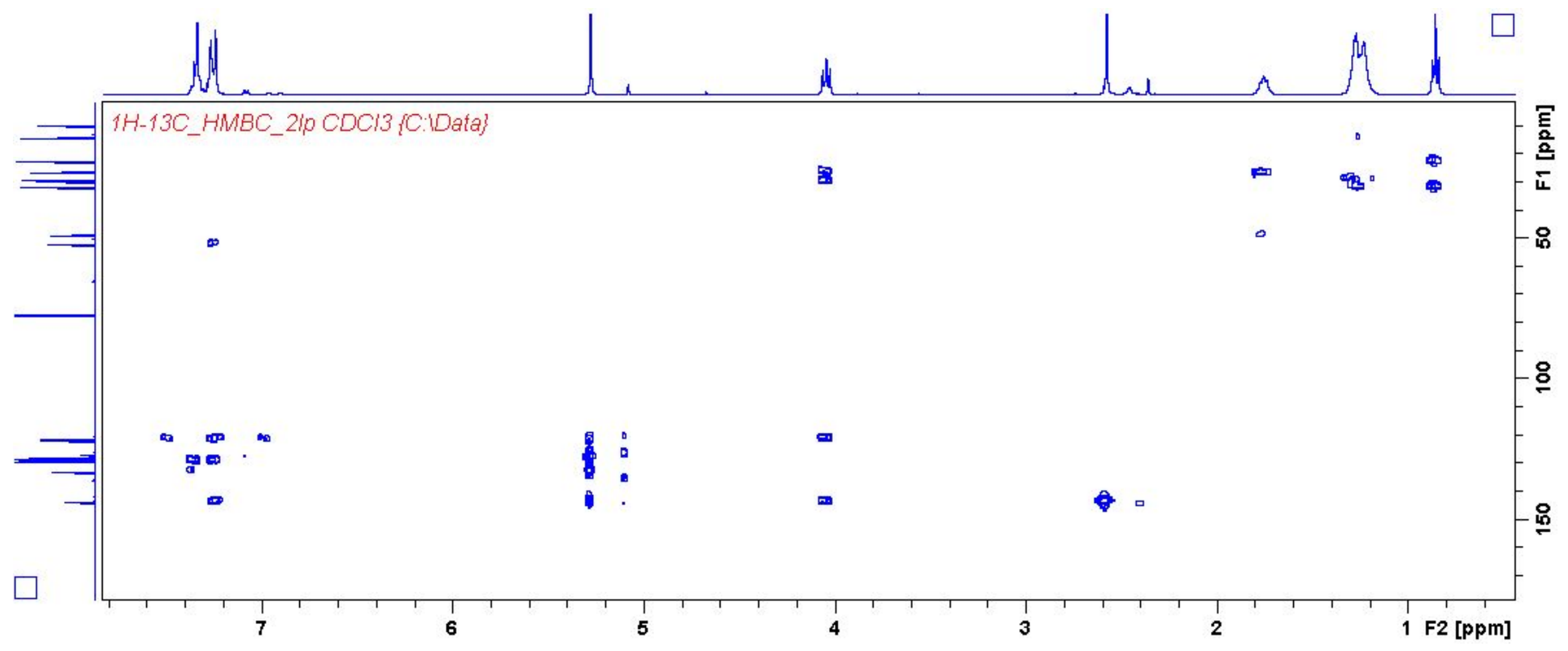


Figure S78. HSQC spectrum for IL product [BMO-im] $\mathrm{BF}_{4}$

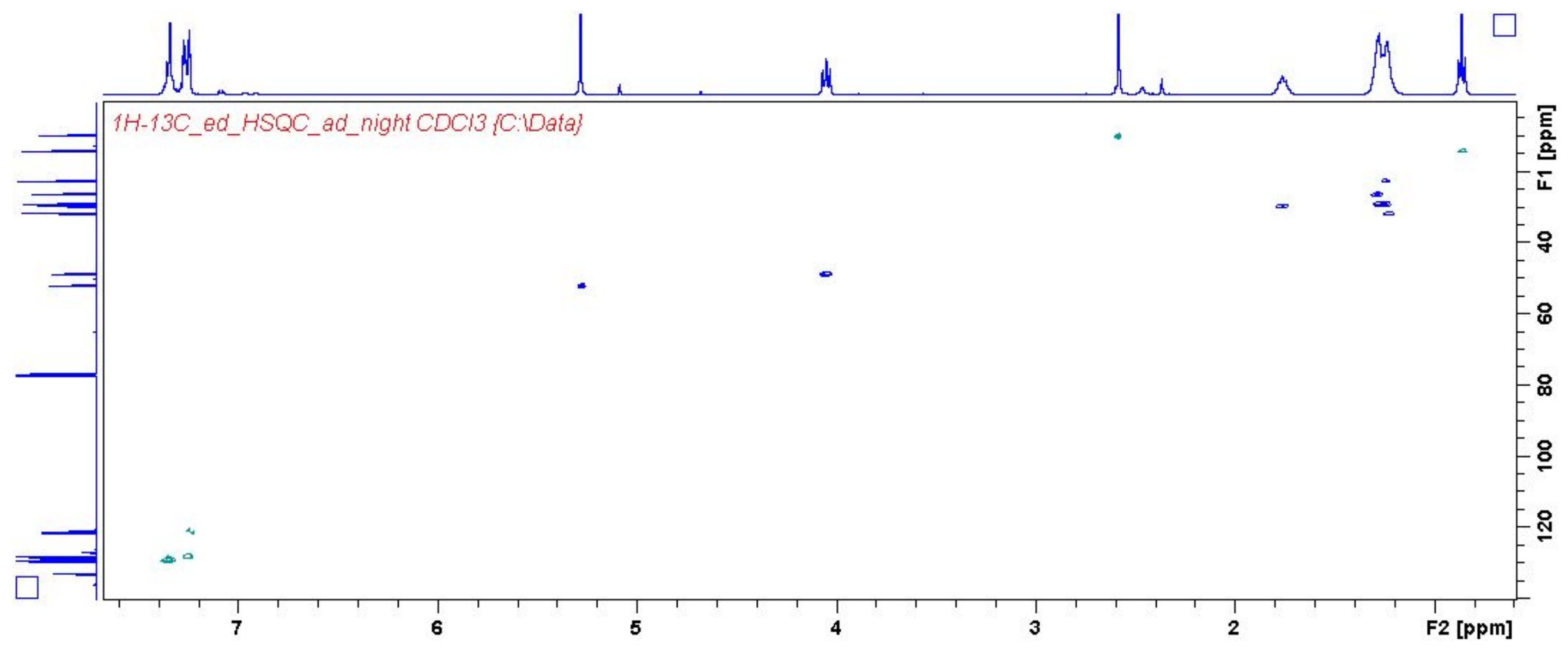

